Portland State University

PDXScholar

\title{
Effect of Tinnitus Maskers on Speech Discrimination Among Those Wearing Tinnitus Maskers
}

John Alexander Chonka

Portland State University

Follow this and additional works at: https://pdxscholar.library.pdx.edu/open_access_etds

Part of the Speech and Hearing Science Commons, and the Speech and Rhetorical Studies Commons Let us know how access to this document benefits you.

Recommended Citation

Chonka, John Alexander, "Effect of Tinnitus Maskers on Speech Discrimination Among Those Wearing Tinnitus Maskers" (1983). Dissertations and Theses. Paper 3244.

https://doi.org/10.15760/etd.3236

This Thesis is brought to you for free and open access. It has been accepted for inclusion in Dissertations and Theses by an authorized administrator of PDXScholar. Please contact us if we can make this document more accessible: pdxscholar@pdx.edu. 
AN ABSTRACT OF THE THESIS OF John Alexander Chonka for the Master of Science in Speech Communication presented February 21, 1983.

Title: Effect of Tinnitus Maskers on Speech Disrimination Among Those Wearing Tinnitus Maskers

APPROVED BY MEMBERS OF THE THDSTS COMMTTTEE.

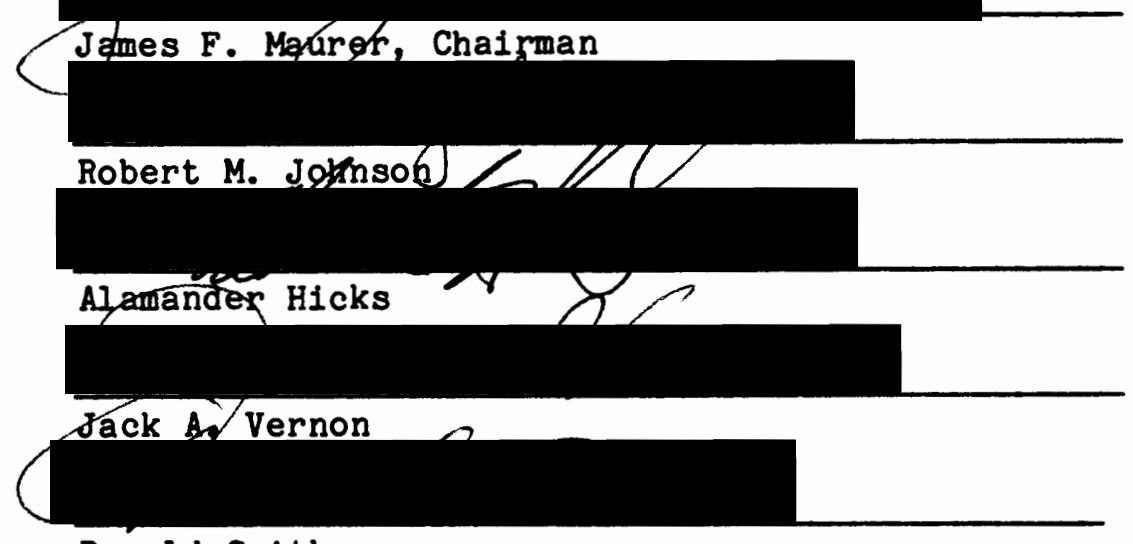

Ronald Smith

The purpose of this study was to determine the effect(s) of tinnitus maskers and tinnitus instruments on speech discrimination utilizing a population of subjects who currently have tinnitus and are presently wearing these devices. The hypothesis which guided this investigation states that there is no difference between discrimination scores with and without tinnitus maskers. In an attempt to test this hypothesis, speech discrimination scores were obtained from 26 listeners both in quiet and in the presence of cafeteria noise, with and without use of their tinnitus maskers.

After pure tone testing and tympanometric screening for possible middle ear pathology, each subject was asked to listen to and repeat monosyllabic words. Prior to speech testing, the subjects were divided 
into two groups according to the type of instrument worn (Audiotone TA-641, Group I and Audiotone TA-640, Group II). Each subject then adjusted his device to a user level and gave verbal responses to tape recorded word lists (Northwestern University Lists, NU-6) presented via a loudspeaker, under six listening conditions; once with no maskers (open ears), once with maskers only, and once with maskers plus amplification. Discrimination scores for each of these conditions were obtained in quiet and in the presence of cafeteria noise. The speech stimuli used in this study was presented at a level $6 \mathrm{~dB}( \pm 2 \mathrm{~dB})$ above the most comfortable listening level for each subject.

In Group I, the within-group comparison of effects between the masker only/no masker (open ears) condition were found to be significant beyond the .05 level of confidence in both quiet and noise. Similar effects for the masker only/masker plus amplification condition were observed with significance reaching the .005 level in quiet and .001 level in noise. However, Group II's overall performance was superior to Group I's, with no significant masker effect observed on speech discrimination while utilizing the TA-640 tinnitus instrument or T-520 tinnitus masker.

Additionally, the combined scores from both groups revealed that reduced discrimination scores were significant for the comparison of effects between the masker only/no masker condition at the .01 and .05 levels of confidence for quiet and noise, respectively. As was the case for Group I, similar effects were observed for the masker only/masker plus amplification, with significance beyond the .005 level of confidence. 
EFFECT OF TINNITUS MASKERS ON SPEECH DISCRIMINATION AMONG THOSE
WEARING TINNITUS MASKERS

\author{
by \\ JOHN ALEXANDER CHONKA
}

A thesis submitted in partial fulfillment of the requirements for the degree of

\author{
MASTER OF SCIENCE \\ in \\ SPEECH \\ with emphasis in Speech Pathology \\ and Audiology
}

Portland State University

1983 
TO THE OFFICE OF GRADUATE STUDIES AND RESEARCH:

The members of the Committee approve the thesis of John Alexander Chonka presented February 24, 1983.

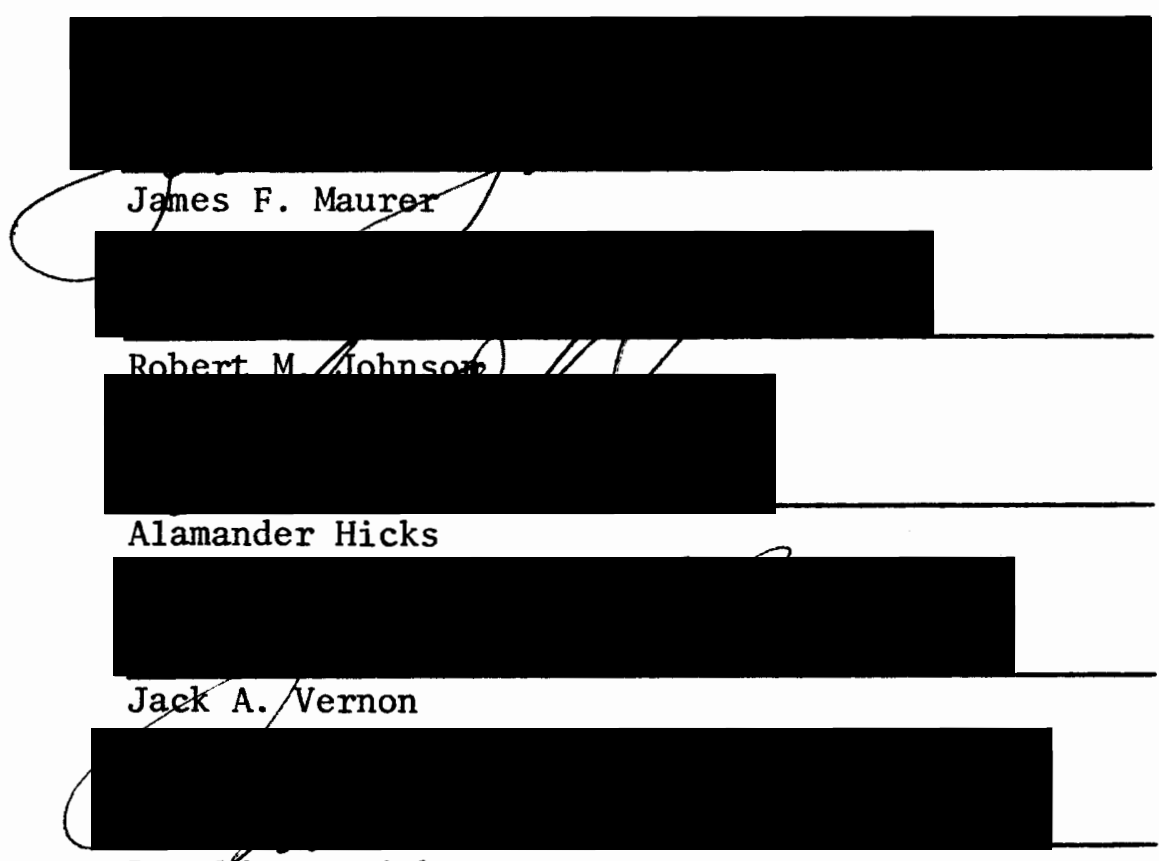

APPROVED:

Ronald E. Smith

Theodore G. Grove, Head, Department of Speech Communication

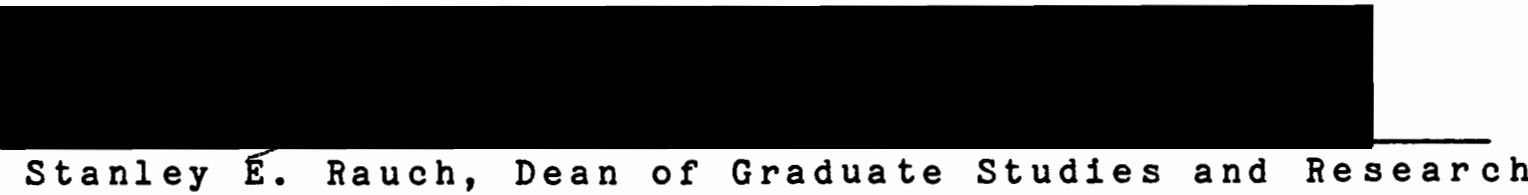




\section{ACKNOWLEDGEMENTS}

This manuscript would not have been completed were it not for the many kind individuals who gave of their time and talents. There is not space enough to express my feelings of gratitude and friendship which I have come to share with these individuals over the past years, but I would like to acknowledge a few who stand out as major contributors in this thesis.

First, to Dr. Robert Johnson goes my deepest expression of gratitude for giving of his time and guidance. Without his expert knowledge of experimental design (for he was a principal figure in planning and implementation of this project), and his many hours of review of this manuscript, its completion would most likely still be in process.

To Dr. Jack Vernon go my sincere thanks for attending my oral defense, and for the use of the research facilities at the Kresge Hearing Research Laboratory, Portland, Oregon. Without this valuable resource and its many kind and tolerant assistants, this project would never have gotten off the ground.

I would also like to extend my deep appreciation to Dr. Ted Grove for his invaluable expertise at synthesizing the world of computers and statistics. His wit and encouragement prevented me from abusing the computer terminal, which more than a dozen times had me at the "default" level of tolerance. 
There is also one man whose constant presence and cheer provided me with the motivation and encouragement to see this project through to the end. I'm referring to my advisor, Dr. James Maurer. He has been a source of inspiration for this exhausted graduate student since my first day on campus. His knowledge of audiology combined with his talents as an educator and professional have instilled in me the highest regard for the profession of audiology. I owe him a great debt.

I would also like to express my feelings of gratitude to a mentor whose knowledge and enchantment of audiology has provided me with the tools which I hope will contribute, in some small way, to the science of hearing. To Dr. Al Hicks, I am deeply grateful.

It would be inappropriate for me not to acknowledge a key person involved in the completion of this study. Without the lightening-fast fingers and keen knowledge of formal scientific writing of my typist, Jill Lilly, this manuscript would most likely still be in a pile on my desk.

Finally, to my wife and family, go my deepest love and devotion for their patience and understanding during this, my loneliest and, by far, finest hour. 
"There is no science that only one man can write."

(Galileo Galilei, 1564-1642) 
TABLE OF CONTENTS

PAGE

ACRNOWLEDGEMENT . . . . . . . . . . . . . . . . . . . 111

DEDICATION . . . . . . . . . . . . . . . . . . . . v v

LIST OF TABLES . . . . . . . . . . . . . . . . . vi11

LIST OF FIGURES . . . . . . . . . . . . . . . . . . . x

CHAPTER

I INTRODUCTION $. . . \cdot . \cdot . \cdot . \cdot . \cdot . \cdot . \cdot . \cdot . \cdot 1$

II REVIEN OF LITERATURE . . . . . . . . . . . . 4

Definition and Incidence.......... 4

Early Observations and Treatment......... 12

Attempts to Measure and Classify Tinnitus . . . . 32

Contemporary Methods of Treatment . . . . . 57

III METHODS ...................... 66

Subjects................. 66

Equipment and Materials ........... 67

Calibration ............... 70

Procedures ............... 73

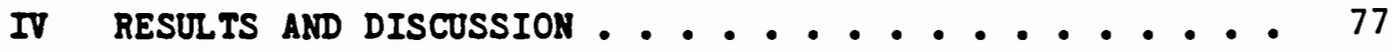

Results with the TA-641 Tinnitus Instrument . . . 78

Results w1th the TA-640 Tinnitus Instrument ... 83

Combined Scores . . . . . . . . . . 90 
vii

v SUMMARY . . . . . . . . . . . . . . . . 94

Conclusion . . . . . . . . . . . . 95

Implications for Further Research . . . . . . 96

REFERENCES . . . . . . . . . . . . . . . . . . . 98

APPENDICES . . . . . . . . . . . . . . . . . . . 105 


\section{LIST OF TABLES}

TABLE

PAGE

I Diagnosis of deafness and incidence of tinnitus . . . 10

II Etiological classifications of hearing loss . . . . . 11

III Etiology of tinnitus (differential diagnosis) . . . . 26

IV Some contemporary measures used in treatment of tinnitus . . . . . . . . . . . . . . . 27

V Response of tinnitus to Vitamin A therapy . . . . . . 29

VI The percentage of pitch matches of tinnitus within frequency ranges of 1000 cps for groups with Meniere's, conductive and sensorineural hearing loss . . . . 38

VII Summary data relating to tinnitus and temporary threshold shift . . . . . . . . . . . . 40

VIII Equivalent (Median) frequencies of noise-induced short-duration tinnitus (NIST) and frequencies of maximum threshold shift (MTS) against stimulus center frequency ........................

IX Results of a follow-up survey of 380 tinnitus patients seen from 1976 through 1978 who were provided specific recommendations to participate in the masking program . . . . . . . . . . . . . . 
$x$ Results of a follow-up survey of 78 tinnitus patients seen in 1979 who were provided specific recommendations to participate in the masking program . . . . . . . . . . . . . . . .

XI Means and standard deviations for speech discrimination scores (in percent correct) of 13 Iisteners wearing the TA-641 tinnitus instrument ( T-570 masker) in quiet and cafeteria noise ......

XII Means and standard deviations for speech discrimination scores (in percent correct) of 13 listeners wearing elther the TA-640 tinnitus instrument or T-520 tinnitus masker in quiet and cafeteria noise ............... 


\section{LIST OF FIGURES}

1. Incidence of tinnitus related to age . . . . . . . 8

2. Schematic illustration of middle ear ligaments and stapedius muscle . . . . . . . . . . . .

3. Schematic illustration of the tensor tympani muscle . . . . . . . . . . . . . . . .

4. Schematic of the stapedius muscle and the attachment of its tendon to the neck of the stapes........

5. The tympanic plexus of nerves: Origin, course and relations . . . . . . . . . . . . . .

6. Origin of the tympanic plexus from the fifth cranial nerve (small superficial N.), the ninth cranial nerve (Jacobson's) and the carotid sympathetic nerves . . . . . . . . . . . . . . . .

7. Cross section of the organ of Corti based on histological and electrophysiological data as well as theoretical considerations . . . . . .

8. Different types of masking curves for tinnitus . . .

9. Acoustic characteristics of masking in relation to the individual's hearing capabilities.......... 
11. Duration of residual inhibition following one minute of masking . . . . . . . . . . . . . . . .

12a. Audiogram and temporal course of masking for internal and external tones... . . . . . . 52

12b. Audiogram and temporal course of masking for internal and external tones . . . . . . . . .

12c. Audiogram and temporal course of masking for

internal and external tones . . . . . . . . . .

13. Frequency response of representative tinnitus maskers . . . . . . . . . . . . . . . .

14. Graphic representation of a spectral analysis of the cafeteria noise utilized in this study .....

15. Frequency response curves for the TA-640 tinnitus instruments . . . . . . . . . . . . . . .

16. Frequency response curves for the TA-641 tinnitus instruments ......................

17. Mean pure tone air conducted threshold responses

for 26 listeners who wear tinnitus instruments . . . 74

18. Histogram displaying discrimination scores for

13 listeners wearing the TA-641 tinnitus

instrument (s) in quiet ................

19. Histogram displaying discrimination scores for 13

listeners wearing the TA-641 tinnitus

instrument(s) in noise ................ 
20. Histogram displaying discrimination scores for 13

listeners wearing the TA-640 tinnitus instrument(s)

or the T-520 masker(s) in quiet ........ 85

21. Histogram displaying discrimination scores for 13

listeners wearing the TA-640 tinnitus instrument(s)

or the T-520 masker(s) in noise ........ 86

22. Histogram comparing means and (standard deviations)

for speech discrimination scores of 26 listeners

wearing either the TA-641 or TA-640 tinnitus

instrument(s) in quiet and cafeteria noise ..... 88 
CHAPTER I

\section{INTRODUCTION}

Tinnitus is the technical term described by Davis and Fowler (1960, p. 91) for "head noises and ringing in the ears." It is a common phenomenon which has been primarily associated with some form of dysfunction along or within the auditory pathway. The multiplicity of anatomical and pathological factors which may contribute to its onset include disorders beginning at the external auditory meatus and extending through the middle ear, the inner ear, the nerve of hearing (N. VIII), and the neuronal connections and fibers along the brain stem to the temporal lobe of the auditory cortex with its many neural radiations to auditory association areas within the brain (Goodhill, 1952). The exact mechanism(s) responsible for its presence among most tinnitus patients has yet to be identified.

Many theories have been proposed detailing hypothetical models for specific causes of tinnitus, but no one theory can be applied universally to all cases. Thus far, the most conclusive etiological findings imply that it is a secondary symptom, the result of some underlying pathological disorder which in many cases is associated with some form of hearing loss (Fowler, 1942; Goodhill, 1950; Myers, 1975; Pennar et al., 1981). However, it has also been shown to be present among those with normal hearing (Fowler, 1912; Wegel, 1931; Hellar \& Bergman, 1953; Riddell, 1956) leading investigators to conclude that 
tinnitus experienced by some individuals may be an early warning sign of declining hearing (Fowler, 1912; Vernon, 1975).

Research attempts at quantifying the acoustical parameters of tinnitus in human subjects have, until recently, been limited to pitch and loudness matching procedures utilizing various methods and techniques. These have varied from the simple matching of an external tone to the loudness and pitch of the internally generated tinnitus to more sophisticated techniques involving masking procedures and reaction time measurements (Feldman, 1971; Goodwin \& Johnson, 1980). Recently, Wilson (1980) has provided additional theoretical evidence for a cochlear origin of tinnitus through use of computer averaged auditory responses (echoes) to acoustic clicks and tone bursts first reported by Kemp (1978).

The past decade has witnessed an increasing number of advances in the area of tinnitus research. Consequently, the audiologist is in a position to contribute to and develop new procedures designed for assessing, referring, and managing those suffering from tinnitus. Furthermore, due to the continuing changes and modifications in measurement and treatment methods for tinnitus patients, the audiologist should have at his disposal an aid to systematically compile audiologic and otologic data concerning the relative effectiveness of various treatments for tinnitus. In view of this, there is a need for additional research concerning the relationship between hearing loss and tinnitus.

Recently, several questions have been raised concerning the lack of systematic data with respect to the relative effectiveness of various 
treatments, and the long term acceptance rates of these methods. specifically, the potential risk to residual hearing and the psychological effects of noise output levels of tinnitus maskers have come under question (Loavenbrack, 1980). One other area that had been virtually unexplored is the effect(s) tinnitus maskers may have on the wearer's speech perception. Only one reported study (Spiczer et al., 1981) has assessed the discrimination ability of non-tinnitus normal hearing subjects while wearing tinnitus maskers in quiet and in cafeteria and white noise. Their results indicated a significant difference in discrimination ability while wearing tinnitus maskers under quiet vs. noise conditions. Specifically, they noted reduced discrimination scores for those wearing tinnitus maskers in the noise conditions, as compared to the no noise conditions.

No reported studies have investigated this parameter utilizing the population for whom tinnitus maskers were designed. Therefore, the purpose of this study was to determine the effect(s) of tinnitus maskers and tinnitus instruments on speech discrimination utilizing a population of subjects who have tinnitus and are wearing these devices. The hypothesis which guided this study states that there will be no difference between discrimination scores with and without maskers. In an attempt to test this hypothesis, speech discrimination scores were obtained from 26 tinnitus patients, both in quiet and in the presence of cafeteria noise, with and without masking.

A brief review of the literature pertaining to this topic will be discussed in the following chapter. 
CHAPTER II

REVIEW OF THE LITERATURE

\section{Definition and Incidence}

Tinnitus is generally considered to be an otologic symptom, a phenomenon common in many diseases of the auditory pathway (Fowler, 1912; Lothrop, 1923; Wegel, 1931; Goodhill, 1952; Graham \& Newby, 1962; Loavenbruck, 1981), as well as a symptom that may or may not accompany other disorders of the body (Fowler, 1944; Riddell, 1956). Its presence should alert the audiologist, otologist, or physician to existing or impending physiological or neurological dysfunction (Gerber \& Mencher, 1980)

The American Heritage Dictionary (1968) defines tinnitus as a sound in the ears, such as buzzing, ringing, whistling or other auditory sensation which is purely subjective. It is derived from the Latin word "tinnire" meaning to "jingle" or "tinkle." Presently, two basic terms are used to identify this widely used expression: tinnitus aurium and tinnitus cranii (tinnitus cerebri).

Tinnitus cranii is a broad term representing a generally non-localized sensation of sound, perceived in the head, which subjectively may be confused or co-exist with tinnitus aurium (Goodhill, 1952; Riddell, 1956; Graham, 1965; Myers, 1975). It is generally more diffuse and may involve psychological or neurological factors which have a mediating role in the perception of the loudness and pitch of tinnitus 
(Goodhill, 1965). Fowler (1912) states that tinnitus of this nature may be the result of mass irritation of the auditory neural elements, caused by rhythmic firings of neural elements discharged by pressure, destruction, or biochemical changes within the central regions of the auditory pathway. Goodhill (1952) and Riddell (1956) go one step further by implicating organic intracranial vascular or neurological disease, thereby removing tinnitus cranii from direct otologic concern, placing it more correctly in the specialty area of the neurologist or neurosurgeon.

Tinnitus aurium can be divided into two unique groups. The first, objective or vibratory tinnitus, is a relatively rare occurrence which can be heard by an observer. This form of tinnitus generally originates peripheral to the inner ear and is believed to stimulate the cochlea via the muscular and/or vascular mediums of the body (Fowler, 1942; Engstrom \& Graf, 1952; Goodhill, 1952; Myers, 1975). The second, and the most common type of tinnitus, subjective or non-vibratory, is an audible perception of sound, present at ear level, which may be mediated anywhere along the auditory pathway by the individual. Theories presented by Fowler $(1942,1948,1965)$ stress that subjective perception of tinnitus aurium should not be considered as an actual sound, but rather an illusion of sound existing only as a sensation. In his extensive works he speculates as to its origins, inferring that it may be a form of biochemical irritation of neural elements contained within the complex workings of the auditory system. He elaborates further by using comparisons to other forms of sensory perception such as itching, tickling, the eye's ability to perceive light in its absence, and the 
ability to taste or smell without corresponding representative stimuli (Fowler, 1942, 1944, 1948).

The incidence of tinnitus is reflected in numerous studies throughout the literature. Although conflicting data as to its prevalence does exist, there is general agreement that it may occur either in the presence or absence of hearing loss (Wegel, 1931; Fowler, 1944; Hellar \& Bergman, 1953; Riddell, 1956).

Fowler (1912), in reviewing 2,000 ear cases, reported that tinnitus was present in two thirds of his patients, and in a subsequent clinica investigation, the same author (1944) reported an incidence of tinnitus among ear patients to be as high as $85 \%$. Jones and knudsen (1928) revealed incidence findings of 59\%, similar to those of Fowler's earlier work. Venters (1953), however, reported markedly different prevalence findings. His survey of 1466 cases of deafness over a 10-year period (1943-52) placed the incidence of tinnitus among his group at only 16.5\%. His rationale for this significant difference from other Investigations was that great advances had been made in otology since the earlier findings of Fowler. In what appeared to be similarly conducted studies, Hellar and Bergman (1953) and Riddell (1956) each reported incidences of tinnitus as high as $73 \%$ among a group of 100 patients with deafness.

Tinnitus is apparently not limited to those with hearing loss or other pathological conditions affecting the auditory system. As early as 1912, Fowler puzzled over the question of why such an acoustically perfect sense organ as the ear was not, in the majority of cases, perceptive of the internal bodily noises which are continuously present. 
He believed that "in every ear there is constantly present a tinnitus..." (Fowler, 1912, p. 702). Subsequent investigators also concluded that practically everyone experiences some form of tinnitus at one time or another (Wegel, 1931; Hellar \& Bergman, 1953; Seltzer, 1947; Riddell, 1956). It was their general belief that people isolated in unusually quiet surroundings could perceive subjective auditory sensations. Indeed, three studies conducted by these investigators (Seltzer, 1947; Hellar \& Bergman, 1953; Riddell, 1956) revealed that 95\% of normal hearing adults experienced some form of tinnitus when placed in a sound treated room (ambient noise levels no greater than $18 \mathrm{~dB}$, re 0.0002 dynes per $\mathrm{cm}^{2}$ ). From a somewhat different perspective, Vernon (1978) reported that a very small percentage of tinnitus patients (8-10\%) seen in the Tinnitus Clinic at the Kresge Hearing Research Laboratory, Portland, Oregon, had normal hearing.

Another aspect of the prevalence of tinnitus can be related to age. Fowler (1948) noted that the incidence of tinnitus was encountered more by those past the fifth decade of life and rose even more rapidly with advanced age. He also observed that children seldom complained of tinnitus. Venters (1953), utilizing a select population from an isolated area of the British Isles recorded the incidence of tinnitus as it related to age, sex and etiology. Figure 1 illustrates that the number of combined cases increases rapidly between the ages of 41-50, dips appreciably at 51-60 and thereafter continues to rise with age. It is interesting to note that the combined pattern is similar to that of the female curve, but the male pattern is much flatter in character. Venters attributed this pattern among males to "overall incidences of 


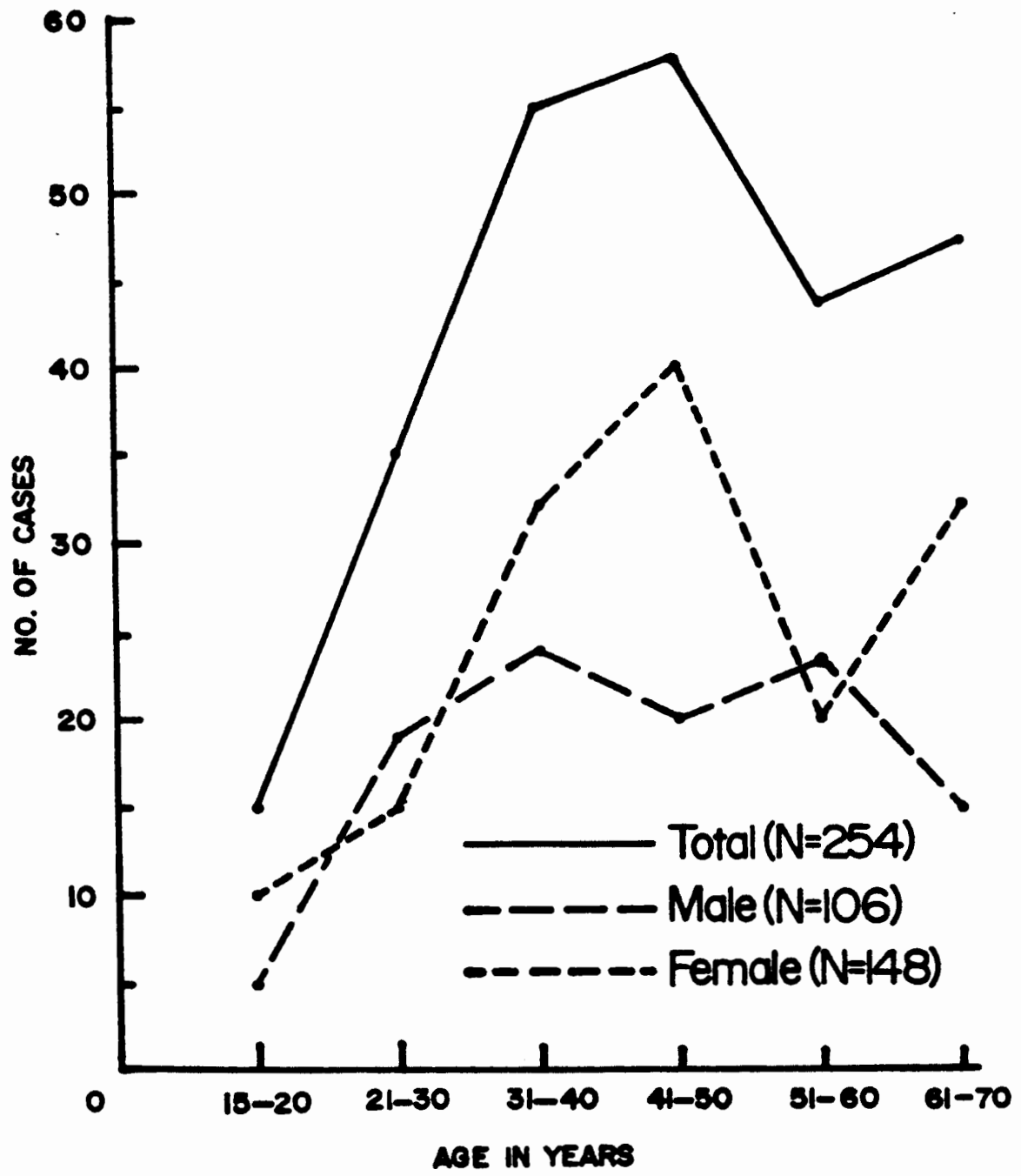

Figure 1. Incidence of tinnitus related to age (from Venters, 1953). 
trauma which are wholey confined to this sex" (Venters, 1953, p. 826). One further point made by Venters concerning the male population in this study revealed that there was no hint of tinnitus related to noise-induced hearing loss. It was his belief that this finding was a result of possible effects from ear protection worn by the population studied as well as possible effects from ears becoming conditioned to increasing levels of noise.

In an attempt to correlate diagnosis of deafness with tinnitus, Hellar and Bergman (1953) reported the incidence of tinnitus among a group of 100 patients to be highest (39\%) in the perceptive deafness category, with the second most prevalent (13\%) in the conductive non-otosclerotic category, and tinnitus due to otosclerosis (8\%) third. However, it should be noted that of the 100 patients in this study, only 8 were diagnosed as having otosclerosis. Of this group, all (100\%) reported some form of tinnitus. Further representation of their findings are displayed in Table $I$.

Fowler (1965), reporting in a series of 2477 otologic cases, revealed similar findings in terms of etiological classification of hearing loss and tinnitus. Table II represents these findings with the most significant percentages appearing in the category of otosclerosis, where $68 \%$ of the cases were reported. This is in general agreement with Hellar and Bergman's (1953) findings of $85 \%$ from a larger group of otosclerotics $(n=83)$ seen in their clinic. However, the sample sizes used in each study were markedly different, and Fowler's study contained a larger number of elderly patients possibly contributing to the large disparities noted in the originally reported studies. 
TABLE I

DIAGNOSIS OF DEAFNESS AND INCIDENCE OF TINNITUS

$N=100$

\begin{tabular}{|c|c|c|}
\hline Diagnosis & $\begin{array}{l}\text { Number of } \\
\text { Patients }\end{array}$ & $\begin{array}{l}\text { Tinnitus Percent } \\
\text { of } 100 \text { Patients }\end{array}$ \\
\hline \multicolumn{3}{|c|}{$\begin{array}{l}\text { Conductive deafness without } \\
\text { otosclerosis }\end{array}$} \\
\hline $\begin{array}{l}\text { Innitus constant } \\
\text { Tinnitus iriconstant } \\
\text { No tinnitus }\end{array}$ & $\begin{array}{r}3 \\
10 \\
7 \\
\end{array}$ & 13 \\
\hline Total & 20 & \\
\hline \multicolumn{3}{|c|}{$\begin{array}{l}\text { otosclerosis ( } 1 \text { case mixed, } \\
7 \text { cases conductive) }\end{array}$} \\
\hline $\begin{array}{l}\text { Tinnitus constant } \\
\text { Tinnitus inconstant } \\
\text { No tinnitus }\end{array}$ & $\begin{array}{l}4 \\
4 \\
0\end{array}$ & 8 \\
\hline Total & 8 & \\
\hline \multicolumn{3}{|l|}{ Perceptive Deafness } \\
\hline $\begin{array}{l}\text { Tinnitus constant } \\
\text { Tinnitus inconstant } \\
\text { No tinnitus }\end{array}$ & $\begin{array}{l}21 \\
18 \\
16 \\
\end{array}$ & 39 \\
\hline Total & 55 & \\
\hline \multicolumn{3}{|l|}{ Mixed Deafness } \\
\hline $\begin{array}{l}\text { Tinnitus constant } \\
\text { Tinnitus inconstant } \\
\text { No tinnitus }\end{array}$ & $\begin{array}{l}2 \\
5 \\
1 \\
\end{array}$ & 7 \\
\hline Total & 8 & \\
\hline \multicolumn{3}{|c|}{$\begin{array}{l}\text { Mixed deafness of one ear, } \\
\text { perceptive of other }\end{array}$} \\
\hline $\begin{array}{l}\text { Tinnitus constant } \\
\text { Tinnitus inconstant } \\
\text { No tinnitus }\end{array}$ & $\begin{array}{l}3 \\
1 \\
1 \\
\end{array}$ & 4 \\
\hline Total & 5 & \\
\hline \multicolumn{3}{|c|}{ Diagnosis not available } \\
\hline $\begin{array}{l}\text { Tinnitus constant } \\
\text { Tinnitus inconstant } \\
\text { wo tinnitus }\end{array}$ & $\begin{array}{l}2 \\
0 \\
2 \\
\end{array}$ & 2 \\
\hline Total & 4 & \\
\hline
\end{tabular}

(From Bellar and Bergman, 1953) 
TABLE II

ETIOLOGICAL CLASSIFICATIONS OF HEARING LOSS

$$
N=2477
$$

\begin{tabular}{lc}
\hline Etiology & Per Cent \\
\hline In otosclerosis & 68 \\
In neural loss & 58 \\
In acute suppuration & 42 \\
In recurrent suppuration & 42 \\
In chronic suppuration & 45 \\
In healed suppuration & 38 \\
In past masked & 54 \\
Average & 56
\end{tabular}

(From Fowler, 1965) 
By now, it should be evident that there is no clear and definitive number which expresses an exact incidence of tinnitus. The latest reported findings of the U.S. Department of Health, Education and Welfare (1968) placed the prevalence of tinnitus in the U.S. at 36 million. of this number, 7.2 million (20\%) were reported to suffer from severe tinnitus. Vernon (1977) feels this figure is no longer accurate, in that the incidence of hearing loss has increased since then, thereby enlarging the prevalence figure for tinnitus as well.

\section{Early Observations and Treatment}

The earliest recorded observations of this auditory phenomenon can be traced back to the writings of Hippocrates. Engstrom and Graf (1952) state that Hippocrates, c.a. 400 B.C., believed that tinnitus was due to auto-ascultation (observing ones own body sounds) of the blood stream. They also reported that Celcus, in the first century A.D., associated tinnitus to head colds, headaches, and epilepsy, and that Galenus, during the period 131 to 201 A.D., described, recorded, and treated incidences of "sonitus in auribus."

In the centuries that followed, diagnosis and treatment of tinnitus was greatly influenced by religious and social superstitions. Treatment for head noises generally consisted of various means of driving out the devil who had entered the body (Myers, 1975).

Myers (1975) describes a paper written by John C. Morgan, in 1877 , which outlined theories and methods of treatment for tinnitus. In it, Morgan states that various etiological factors and available medica remedies for tinnitus had changed little throughout the ages. 
Consequently, he proclaimed that tinnitus was a major otologic problem which appeared most embarrassing to the medical profession.

Graham (1965) recounts a mixture of modern and ancient practices of medicine in the writings of John Nottingham, a medical text writer during the mid-1800s. In 1857, Nottingham used a relatively scientific approach in describing tinnitus. He stated that tinnitus is, "met with in cases where diseased conditions tell upon, or extraneous matters interfere with, the membrane tympanil, the eustachian tube, the ossicula, or the chorda tympani nerve." He was also aware of what is now termed subjective tinnitus and its relationship to the inner ear. The intriguing "ancient" aspect of this account lies in his advocacy of the use of leeches as a treatment for tinnitus.

As a result of the shortage of empirical data regarding tinnitus during this period, numerous theories were offered as an alternative. By the end of the 19th century, efforts to better understand its origins and pathogenesis had progressed no further than a growing compilation of various cases and their assorted methods of treatment (Graham, 1965). During this period, the site of lesion of tinnitus was belleved to lie in the middle ear (Graham, 1965).

Following World War I, efforts to increase the volume of knowledge concerning tinnitus led to more tenable theories attempting to explain the mechanism(s) of middle ear involvement. A theory developed by Lothrop (1923), which today is still applicable in certain cases, concerned the abnormal displacement of the ossicular chain. Normally, the structures housed in the middle ear are held in a state of equilibrium by the tensor tympani and stapedius muscles along with their 
IIgaments and attachments (see Figures 2 to 4 ). The physical properties of inertia and elasticity within this system play a key role in normal movement of the ossicular chain and its subsequent transmission of sound to the inner ear (Durrant \& Lovinic, 1977). Lothrop (1923), hypothesized that,

if pathological conditions supervene and cause a displacement of the tympanic membrane, ossicles, and ligaments, or any adhesions form involving the sound conducting apparatus, an abnormal position of the tensor tympani and stapedius muscles must result and the normal balance is destroyed.

One such pathological condition involving adhesions of the sound conducting apparatus is otosclerosis. This is a condition generally characterized by an abnormal bony growth originating in one of the three small bones (malleus, incus, stapes), usually the footplate of the stapes, which are housed in the middle ear (see Figures 2 \& 4). The work of Saltzman and Ersner (1949) lends support to Lothrop's theory in that their observations revealed a lack of equilibrium in the middle ear due to an abnormal position or restriction of movement of both tensor tympani and stapedius muscles in otosclerotic patients.

Other early observations involving the middle ear implicate the tympanic plexus as the mediating factor in tinnitus (Lempert, 1940; Schnider, 1947; Trowbridge, 1949). The tympanic plexus is a small network of nerves located on the promontory of the medial wall of the middle ear (see Figures $5 \& 6$ ). According to these authors, its composition and role in tinnitus involves primarily the 5 th (trigeminal), 7 th (facial), and 9 th (glossopharyngeal) cranial nerves. Trowbridge (1949) believed that tinnitus was produced by pathologic changes in the middle ear and neighboring structures such as the 


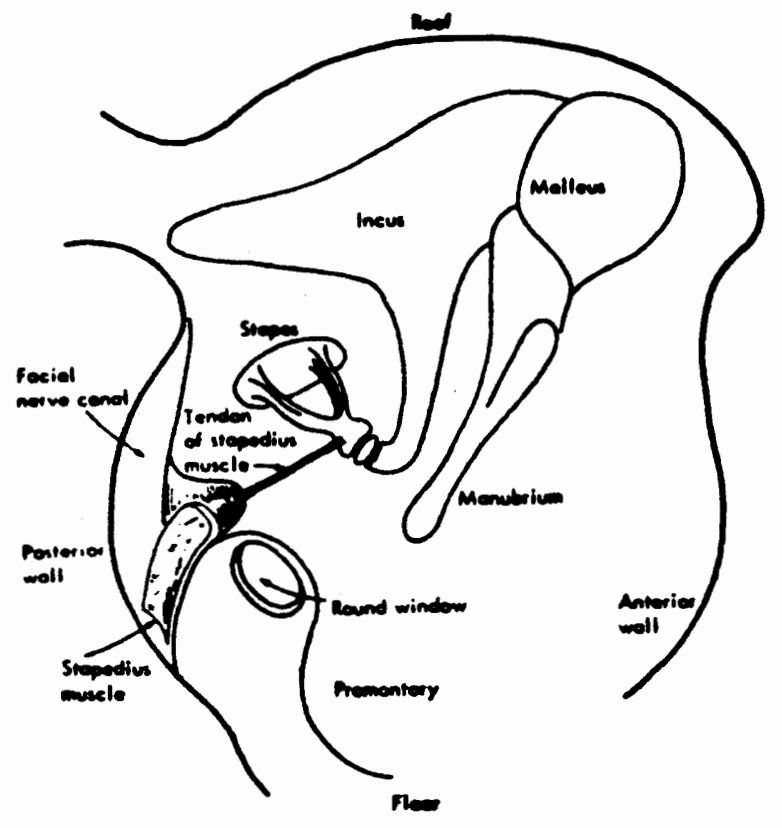

Figure 2. Schematic illustration of middle ear ligaments and stapedius muscle. (From Zemlin, 1968)

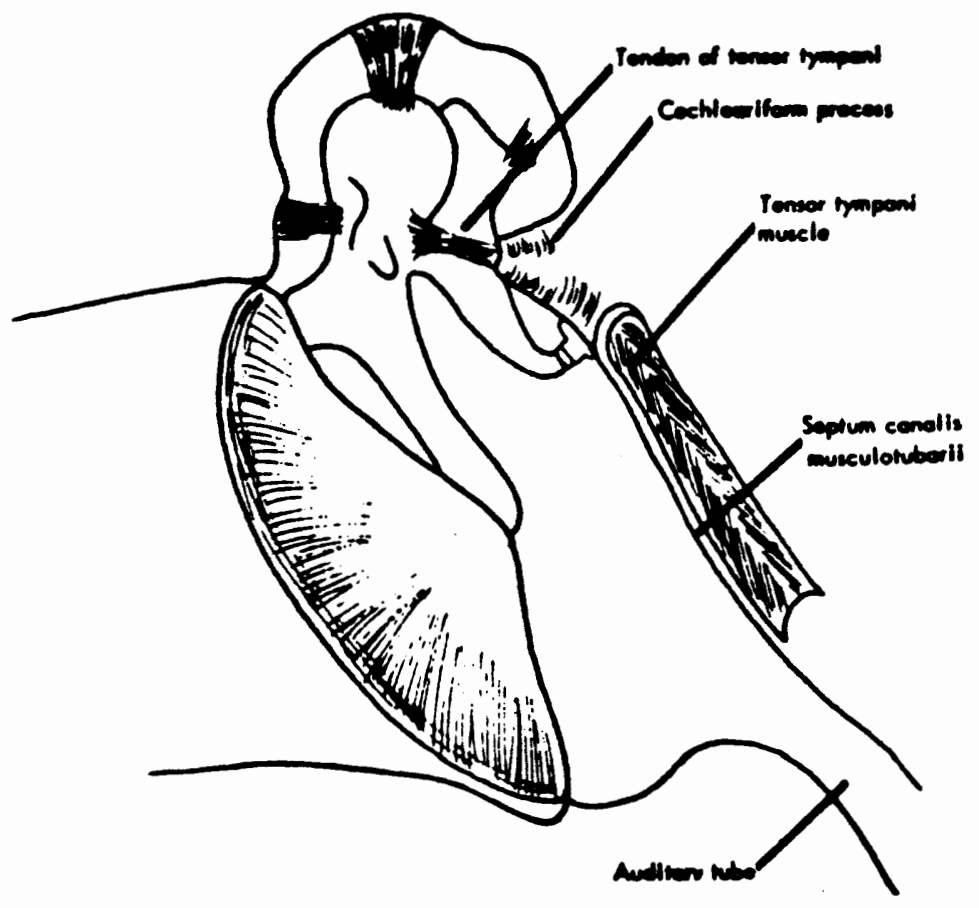

Figure 3. Schematic 1llustration of the tensor tympant muscle (From Zemlin, 1968) 


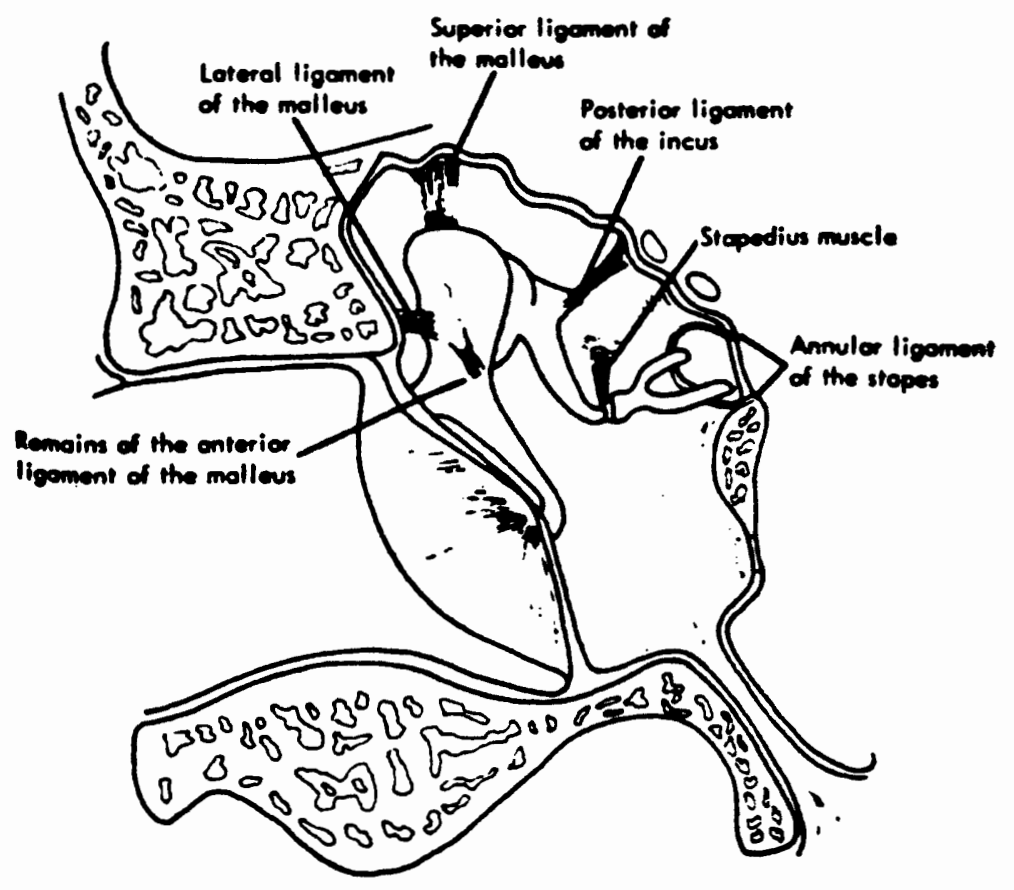

Figure 4. Schenatic of the stapedius muscle and the attachment of its tencion to the neck of the stapes. (From Zemlin, 1968). 


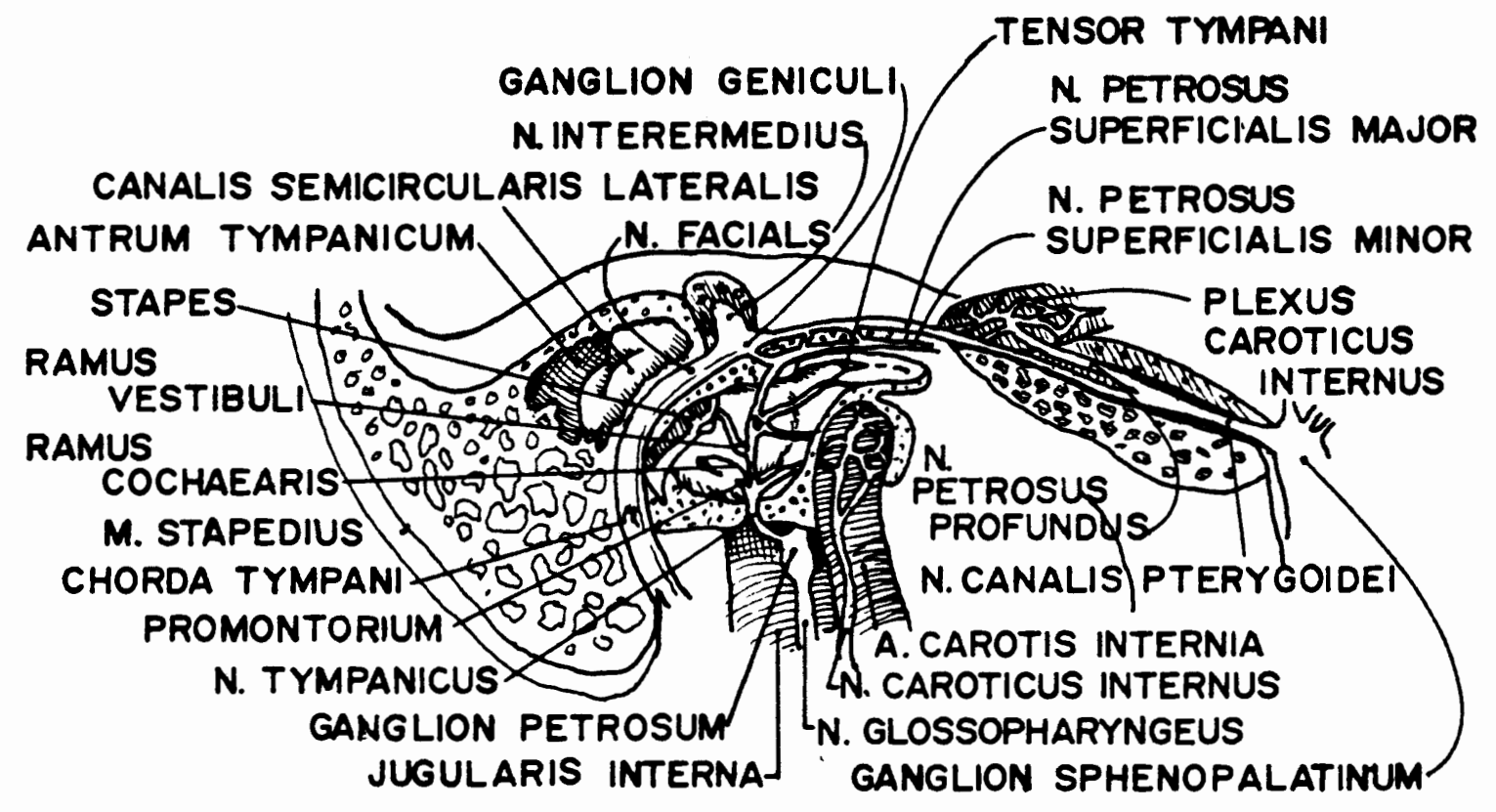

Figure 5. The tympanic plexus of nerves: Origin, course and relations. (From Lempert, 1946). 


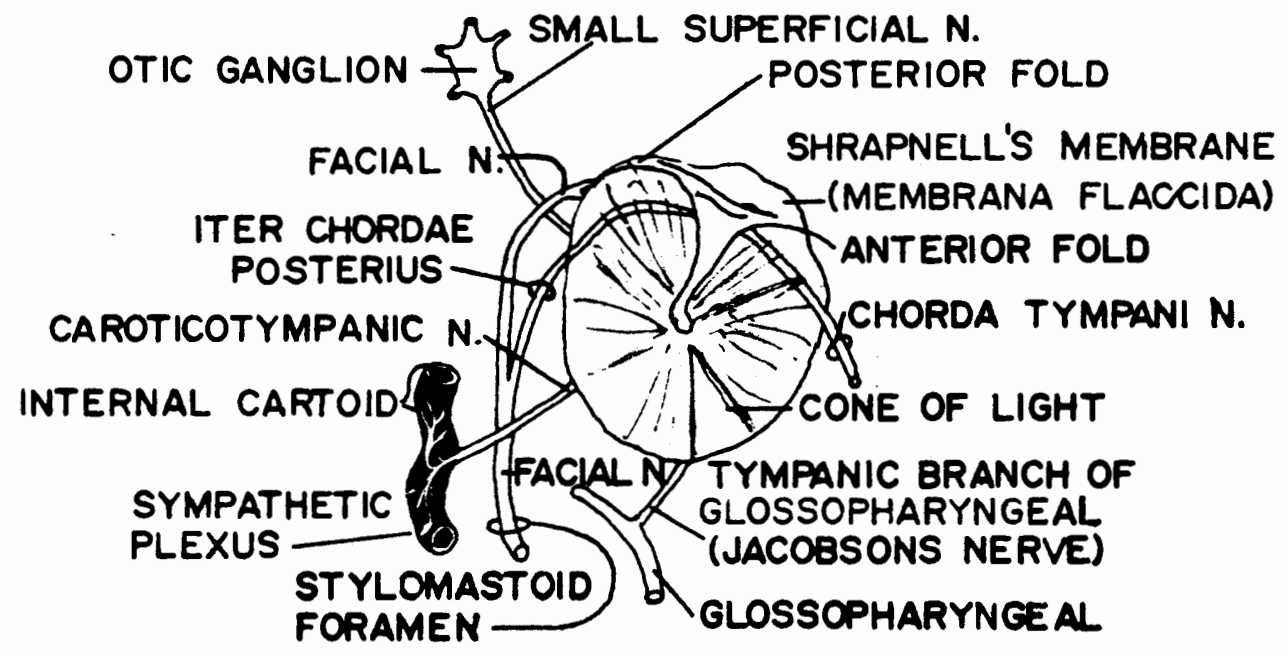

Figure 6. Origin of the tympanic plexus from the fifth cranial nerve (small superficial N.), the ninth cranial nerve (Jacobson's) and the carotid sympathetic nerves.

(From Trowbridge, 1949). 
pharynx, nose, and eustachian tube, and was transmitted through the tympanic plexus which is connected to the cochlear neuromechanism of the Inner ear.

Schnider (1947) presented an elaborate theory in which he details the intricate interconnections between the balance, vegative, and emotional mechanisms with the hearing apparatus. It is a complicated theory, providing two routes for sound in hearing: an external (primary) pathway, and an internal (secondary) pathway, uniting neural elements from the teeth and upper resonating cavities of the skull with the labyrinth, cochlea, and the brainstem. It is Schnider's contention that this internal system may at times bypass the primary auditory pathway (N. VIII) at the peripheral level, and transmit sound to the cochlea and brainstem via the afferent fibers of the tympanic plexus. This interaction between the internal (non-auditory) and external (auditory) pathways form the basis for his tympanic plexus theory of hearing. According to Schnider's model, the internal system acts as a support for the external system, both transmitting acoustic information from the environment and together are mediated cortically as one wave form.

Moving from the middle to the inner and more central structures of the hearing mechanism, we find theories becoming more elaborate and complex as knowledge and sophistication concerning the auditory system grows.

Jones and Knudsen (1928), in the case of cochlear lesions, proposed a theory based on physiological evidence gained from studies of neuronal activity. They concluded that any lesion interfering with the excitation of an appropriate number of normal neurons, would result in 
impaired hearing. The impairment was thought to be a result of imperfect articulation between the tectorial membrane and the nerve endings (see Figure 7). In the case of tinnitus, they felt that the articulation between the membrane and the nerve endings was more permanent, resulting in a continuous excitation of nerve endings.

Hamblen-Thomas (1938) has also reported on possible changes in the inner ear which may contribute to the cause of tinnitus. He implicates apparent arterial or venous dysfunction by citing many cases in which blood flow to the VIII nerve or the cochlea is disrupted or altered. Furthermore, he suggests that since in the majority of cases tinnitus does not appear to fatigue, it is the VIII nerve that may be involved. It was his contention that the normal inhibiting state of the VIII nerve, in which it does not respond to small, subliminal normal Impulses, breaks down in the presence of trauma and the nerve becomes hypersensitive, thus receiving impulses (tinnitus) which would not normally affect hearing.

Along a somewhat similar line of reasoning, Stevens and Davis (1938) state that,

the most satisfactory explanation of most cases of ringing in the ears called tinnitus is that certain hair cells, or nerve fibers connected with them, become hyperirritable and discharge nerve impulses more or less continuously as a result of some pathological process. This abnormal condition may be acute or temporary, as a result of excessive stimulation by a loud sound, or it may be chronic.

It was Fowler (1944) who stated that all head noises are the result of the afferent (sensory) portion of the auditory tract. He had previously (1941) described tinnitus of cochlear origin as a "line busy" phenomenon. In this description, he stated that tinnitus was always 


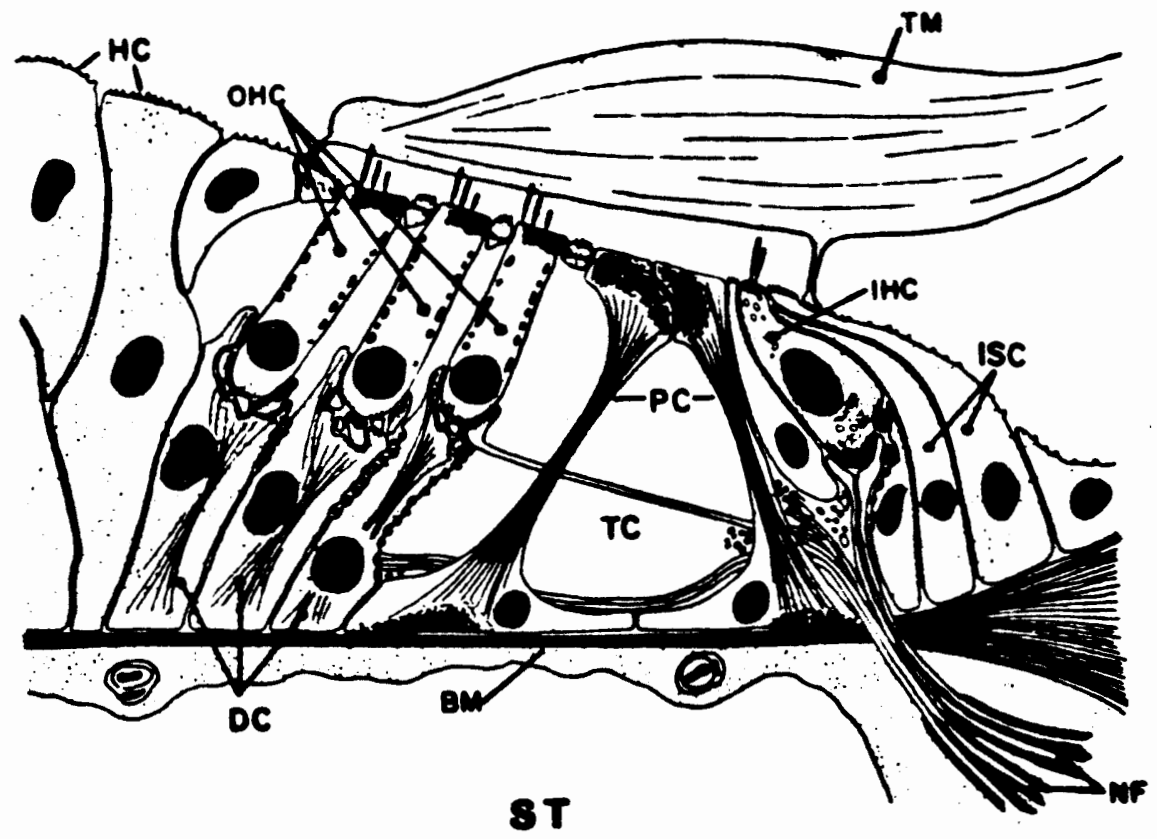

Figure 7. Drawing of the cross-section of the organ of Corti, based on histological data, including electron microscopy, as well as electrophysiological data and theoretical considerations. Scala media (SM), tunnel or Corti (TC), scala tympani (ST), tectorial membrane (TM), inner supporting cells (ISC), Deiter's cells (DC), Hensen's cells (HC), pillar cells (PC), nerve fibers (NF), and outer (OHC) and inner hair cells (IHC). (From Durrant \& Lovrinic, 1977). 
associated with hearing loss and that deafness occurs because the neurons producing the tinnitus were already "busy" and could not be activated by external sound sources. However, Fowler (1944) later made the observation that some of his patients were complaining of tinnitus, yet showed no evidence of hearing loss or other ear pathology.

From a similar perspective, Atkinson (1944) viewed tinnitus as an auditory sensation in which the nerve endings of the cochlea were stimulated in a manner identical to nerve endings on other areas of the body. That is, he regarded tinnitus as being similar to any form of paresthesia (spontaneous sensation) experienced by the peripheral sensory system such as itching, tickling, etc.

Other theories of tinnitus involving the neurovascular supply to the auditory system have been proposed. Fowler and Fowler (1950) Implicated the cochlear vascular supply as the mediating factor in tinnitus, stating that emotional state was a probable contributing cause, its effects leading to reduced blood supply to the cochlea in much the same manner as trauma. They stated that, nafter shock. - and in all severe infections and toxic diseases, thick agglutinated masses (sludges) have been observed to form in the blood" (Fowler \& Fowler, 1950, p. 925). Further, they felt that in most cases the larger the vessels the less likely the chance of being affected by these sludges. However, the vessels which supply the cochlea are extremely small, particularly in the region of the stria vascularis. Consequently, its neural elements and supporting structures are more prone to insult and reduced blood flow. 
Fowler and Fowler (1950) stressed three factors which they felt may be important in determining whether blood sludging was indeed a contributing cause of tinnitus. First, the chemical make-up of the sludging agent(s) must be identified and correlated with the production of tinnitus. Second, the maximum length of time such agent(s) can remain in the cochlear blood supply before harmful side effects are observed must be determined. And finally, the health history of the individual must be considered in determining overall susceptibility to ear disease.

Thus far, theories of tinnitus have centered on symptoms leading to organic disorders. In viewing tinnitus from a psychological orientation, Kennedy (1953) proposed for otologists a classification of psychiatric patients complaining of tinnitus. The first of these categories he labeled depressive preoccupation with tinnitus; since depression is a common psychiatric diagnosis, the patient with tinnitus may become preoccupied with the internal head noises to such an extent that he rationalizes its presence as the cause of his depression. In the second category, tinnitus is viewed as a symptom of psychoneurotic escape; in occupations with high noise hazards, workers may experience tinnitus related to their job. For those individuals who do not understand or are afraid of their job there is the possibility that the normal psychological suppression of the work-related high-intensity noise will not occur. The presence of tinnitus was believed to possibly serve as the unconscious reason for a lack of self-respect related to job performance. The third category dealt with anxiety states--psychosomatic tinnitus; this category included those who felt 
anxious prior to a stressful or dangerous event. When there was persistent anxiety due to personal, unresolved problems or frustrations it was believed to be mediated in the nervous system with tinnitus being a prominent symptom. A fourth classification consisted of pre-senile, senile and arteriopathic deterioration; among these the pattern of cerebral disorder varied from cortical atrophy to a more generalized degeneration similar to the Alzheimer type. It was noted in these patients that as the disease process advanced tinnitus became less disturbing. And finally, the fifth category, malignant hypochondriasis, deals with those who are preoccupied with some minor infirmity. Kennedy believed that this condition could lead to a gradual and serious deterioration of personality. He states that when tinnitus is the presenting symptom surgical attempts at relieving the problem are not recommended.

In his overall findings among psychiatric patients complaining of tinnitus, Kennedy (1953) concluded that the major factor contributing to the distressing perception of tinnitus was related to the extent of the patient's preoccupation and frame of mind concerning the tinnitus. He stressed that before operative treatment was contemplated the use of medication and/or psychiatric methods be employed in an effort to reduce or eliminate the patient's distress from tinnitus.

Fowler and Fowler (1950) also believed that there was some form of psychological "effect" or "feeling tone" which accompanied all sensation, including tinnitus. It was their contention that failure to act or overacting emotionally to the environment was the result of some functionally mediated disorder. The emotional responses were believed 
to be primarily responsible for time of onset, duration, severity, recurrence, variation, and ultimately the outcome of various diseases, tinnitus being a symptom playing a role in psychological or emotional etiology.

Treatment for tinnitus during this early phase of its investigation generally encompassed a wide variety of methods based on differing opinions as to etiology and diagnosis. Fowler (1942) believed that diagnosis of tinnitus aurium required careful examination to determine etiology, contributory causes, and prognosis, along with much effort at revealing a common site of lesion. In a subsequent investigation (1948), he stated that there would never be one "cure" because no single cause exists.

Graham (1965) stresses that treatment of any disease or pathology should be based on an understanding of the dynamics of that system. When many pathophysiological mechanisms are or can be suspect, alone or in combination, a single cure would be highly unlikely (see Table III). This is the situation which he feels is present with regards to tinnitus of a non-vibratory nature. An ideal approach to treatment might involve a therapeutic assault on the related etiological factors present (Hellar \& Bergman, 1953).

Some contemporary measures which have been used for treatment of tinnitus are presented in Table IV. It was Fowler's (1944) belief that the structures of the ear should not be viewed as independent entities, but rather as intimately connected parts of a whole that are frequently influenced by each other. Some of the most popular therapies used in treatment of tinnitus in the past have been evaluated for effectiveness 
TABLE III

ETIOLOGY OF TINNITUS (DIFFERENTIAI DIAGNOSIS)

ANATOMIC LOCATIO
EXTERNAL AUDITORY CANA
Tympanum
a. Tympanic membrane
b. Tympanic muscles
c. Ossicles
d. Tymp. plexus
e. Tymp. vessels
f. Eustachian tube
g. Fenestrae--Rotund

PATHOLOGIC LESION

Cochlea
a. Perilymph
Neuronitus
b. Endolymph
Necrosis
c. Organ of Corti
Fibrosis

VIIIth Nerve
a. Spiral ganglia
b. Trunk
Tumor
Aneurysm
Capillary fragility

Intracerebral
a. Ventral and dorsal nuclei
b. 2nd order neurons
c. Medial geniculate body
d. 3rd order neurons
e. Cortex

Stasis

sludging

Vascular spasm

Vascular sclerosis

vasomotor paralysis

(From Goodhill, 1950) 
TABLE IV

SOME CONTEMPORARY MEASURES USED IN TREATMENT OF TINNITUS

MEDICAL:

1. Medication: bromides, barbiturates, other sedatives, potassium iodide, vitamins, benzyl cinnamate, antiallergic drugs, histamine therapy, intravenous procaine.

2. Local therapy to disease processes.

3. Elimination of drugs and intoxicants

4. Elimination of foci of infection

5. Correction of faulty gastrointestinal function

6. Correction of metabolic diseases.

7. Control of diseases of the vascular system and blood-forming organs

8. Dietary control of fluids, salt, and water balance.

9. Dental rehabilitation.

10. Intratympanic medication.

11. Therapy directed to correct nose and throat pathology, including roentgen and radium therapy.

12. Politzerization, inflation, message.

13. Removal of cerumen.

14. Psychotherapy.

15. Hearing aid.

16. Electrical therapies, i.e., ultraviolet, quartz lamps, galvanism.

SURGICAL:

1. Otologic:

ossiculectomy

mastoidectorny

tympano-sympathectomy

fenestration of the labyrinth

obliteration of the saccus endolymphaticus

2. Rhinologic

3. Spinal tap

4. Cranial surgery for tumor, vascular anomalies, section of the eighth cranial nerve.

5. Splanchnectomy and similar techniques for alleviation of hypertension.

(From Hellar and Bergman, 1953) 
and are presented below.

One area of investigation that has occurred in the realm of medical treatment involves the use of Vitamin A. Animal studies have shown that diets lacking vitamin A can cause cochlear degeneration, and that massive doses of the vitamin will arrest the condition (Graham, 1965). Lobel (1949) found that it was also effective in improving hearing, as well as relieving tinnitus in humans. Subsequent studies by Anderson et al. (1950) and Bau and Savitt (1951) agreed well with these results. However, further investigations by Baron (1951) and Atkinson (1954) failed to substantiate those findings. Table $V$ shows the results reported by these investigators.

Numerous other drugs have been used with some reported success, but are generally inconsistent in producing positive results. Based on anatomic, histologic, and clinical observations, Trowbridge (1949) injected the tympanum with ethylmorphine hydrochloride, an analgesic anesthetic. He stated that its therapeutic value as an effective treatment for tinnitus, in properly selected cases, was due to its stimulation of the vascular and lymphatic supply of the tympanic plexus. Fowler and Fowler (1950) also reported some success at reducing tinnitus in psychosomatic patients exhibiting blood sludging with use of procaine.

Some success at treating tinnitus has been obtained through the use of nicotinic acid. Flottorp and Wille (1955) reported that daily use of the drug in carefully prescribed doses has been beneficial to certain groups of tinnitus patients classified through audiological measures. Their findings indicate that the vasodilating effects of the drug are 
TABLE V

RFSPONSE OF TINNITUS TO VITAMIN A THERAPY

$\begin{array}{ccc}\text { Investigator } & \begin{array}{c}\text { Number of } \\ \text { Cases }\end{array} & \begin{array}{c}\text { Improvement in } \\ \text { Tinnitus }\end{array} \\ \text { 1. Lobel } & \text { "about" } 300 & \text { "considerable } \\ \text { number" }\end{array}$

2. Andersson, Zoller, and Alexander

23

9

17

10
73.98

66.68

23.58

none

(From Graham, 1965) 
most effective in treatment of tinnitus associated with high frequency, cryptogenic, and menieriform hearing losses. Atkinson (1944) has also reported on this method of treatment with the best results occurring among those with conductive type hearing loss.

Hatton et al. (1960) has reported on the use of galvanic current for relief from tinnitus. They used a method which reversed the polarity of electrical current by placing either the anode or cathode on the zygomatic arch of the tinnitus side. When an anode placement was used, they observed a decrease in tinnitus as the current was increased. They believed that the galvanic current was affecting some regulatory mechanism within the nerve fiber being stimulated.

Two general surgical approaches were advocated in specific cases of tinnitus during these early stages of investigation. The relationship between the sympathetic system and vasodilation or vasoconstriction prompted Passe (1951) to use a stellate ganglia or upper dorsal sympathetic ganglia block for relief from tinnitus. Johnson (1954) has also used stellate block as a precursor to dorsal sympathectomy for reduction of tinnitus. Lempert (1946) and Schnider (1947) both advocated stripping of the tympanic plexus (tympanosympathectomy) in cases where this neural network has been damaged or was malfunctioning.

Other types of treatment used to reduce the effects of tinnitus involve psychotherapeutic measures. Treatment of this type was often used to supplement medical or surgical intervention. Goodhill (1954) stresses that in cases of subjective phenomena, a double diagnosis must always be considered: 1) the actual organic etiological otologic lesion, and 2) the psychosomatic status of the patient. The latter 
takes into account both the emotional threshold of the patient, as well as any specific anxiety states or phobias. Following this double diagnosis in which decompensated tinnitus (recognized as a problem by the patient) is present, Goodhill (1954) lists six measures for the treatment of tinnitus:

1) Specific otologic Therapy--The organic otologic disease focus must be approached with the idea of therapy directed specifically to the eradication of the tinnitus.

2) Palliative Neasures--Simple reassurance as to the reality of the tinnitus accompanied by encouragement and good prognosis will go far in helping alleviate the anxieties of the patient with decompensated tinnitus.

3) Sound Sedation--Sound sedation is very help-lil in many cases of tinnitus, especially in regard to the difficulty in sleep, which is a great problem with many patients. . The use of a bedside or pillow radio or phonograph speaker is very helpful in providing an artificial source of ambient noise to mask out the subjective tinnitus.

4) Drug Sedation--Drug sedation is an important palliative procedure, not only for daytime use but especially for bedtime tinnitus irritability. . No one drug should be used for any long periods.

5) Surface. Psychotherapy--. - In cases where decompensated tinnitus is accompanied by major psychologic stresses and strains, a certain degree of psychotherapy. . Is helpful. Such surface psychotherapy should include a thorough explanation as to the real nature of the tinnitus with assurance that it is neither an hallucination nor an illusion.

6) Major Psychotherapy--Deep psychotherapy in psychiatric hands is indicated in every case of orsidized, symbolic, verbal, or musical tinnitus. It is also indicated where surface psychotherapy. . . has not solved the decompensated tinnitus problem... 
Through these measures, Goodhill (1954) feels that efforts will not be directed so much toward a cure for tinnitus as to the more important goal of making it more tolerable.

One final means of tinnitus management involves the use of amplification. It was Saltzman and Ersner (1947) who discovered that use of a hearing aid was effective in relieving tinnitus. Through proper fitting of a hearing aid, in certain cases, sufficient amplification of ambient noise resulted in reduction or masking out of the tinnitus. However, relief through amplification was precluded for many patients due to the nature and/or extent of their hearing loss.

Up to this point, most of the accumulated research and clinical data have been based on theoretical interpretations of observations made on the auditory mechanism and its role in perception of tinnitus. With the advent of the audiometer (early 1920's) and development of more sophisticated electronic equipment, the ability to make accurate measurements enhanced our knowledge of the auditory system and tinnitus.

\section{Attempts to Measure and Classify Tinnitus}

Early attempts to measure tinnitus were somewhat limited by the subjective nature of the symptom, as well as its elusive site of lesion which can be located anywhere along the auditory pathway. This limitation has compounded the difficulty of human study, by virtue of the inaccessibility to direct measures of the inner ear and central portions of the auditory pathway among living subjects (Reed, 1960). As a result, researchers have been forced to develop and utilize indirect measures in their study of tinnitus. 
In reviewing the literature, Graham (1965) reports one of the earliest recorded accounts outlining an attempt to measure the physical parameters of tinnitus. He states that Cowan, in 1897, described a patient with tinnitus located in the frequency region of 500 to 700 cycles per second (cps) and of variable loudness. Later, with the advent of the first electric audiometer, audiometric measurements of tinnitus began to appear.

Wegel (1931) made audiologic measurements of his own ear in a study which he termed "the Abnormal Sensation Areas". He found that his own tinnitus was not very loud and most closely approximated a tone of 3600 or 3700 cps. In an effort to determine which frequency and intensity levels would mask out his tinnitus, Wegel (1931) plotted masking curves for frequencies 100 through $15,000 \mathrm{cps}$. He noted that increasing the intensity of a 3400 cps tone resulted in a marked change in the character of his tinnitus at threshold. When the external tone was combined with the tinnitus ear, a definite discord was produced. At intensities above this discord area, his tinnitus was entirely masked out and only a pure tone was perceived. Wegel noted further that as the external tone approximated $3620 \mathrm{cps,} \mathrm{there} \mathrm{appeared} \mathrm{an} \mathrm{unmistakable}$ "beat" at threshold between the tone and his tinnitus. Additionally, he observed that as the tone was slowly decreased in pitch, the perceived beats became faster at or near threshold, ending in the discord described at $3400 \mathrm{cps}$. When the pitch of the tone was at a certain region above 3620 cps, fast beats reappeared at threshold, but when intensity was increased sufficiently, a cancellation of both tone and tinnitus occurred. He observed that tones between 3200 and $3600 \mathrm{cps}$ 
with a specific amount of intensity would not only render the tinnitus inaudible, but also extinguish itself. Wegel reasoned that the perceived beats were the result of interference in generating nerve impulses between the periodic tone and autoperiodic chemical or electric stimulus of the tinnitus.

Josephson (1931) proposed and developed a method for measuring the pitch and loudness of tinnitus. Using a beat frequency oscillator and a vacuum tube voltmeter, he determined that the majority of his subjects displayed a frequency of tinnitus generally located in the region where the auditory curve drops off sharply (as did Wegel, 1931). Furthermore, he observed that in cases of moderately advanced deafness, high-pitched tinnitus was usually present at frequencies between 2500 and 4000 cps. Low-pitched tinnitus was observed to occur most of ten in the frequency region of 40 to $200 \mathrm{cps}$. In addition, he noted that the tinnitus was drowned out (masked) by increasing the intensity of the external tone. Moreover, he could elicit this masking phenomenon either ipsilaterally or contralaterally at sufficient intensity, and in some cases, the tinnitus could be inhibited for a certain period of time after the stimulus had ceased.

In an attempt to correlate audiometric configuration and pitch of tinnitus, Fowler (1940) noted that tinnitus usually occurred along the upper portions of the sides of the hearing loss trough, closer to where the healthy and degenerated elements meld. The frequency side of the trough exhibiting the tinnitus frequency was believed to depend upon the severity and spacial distribution of the lesion. Audiograms displaying a precipitous dip in hearing generally had tinnitus located near the 
"brow of the hill". Those with a more shallow, less severe loss tended to show a tinnitus frequency region closer to the bottom of the trough. These findings were in general agreement with those of Wegel (1931) and Josephson (1931) and more recently with Loeb and Smith (1967).

In a subsequent study, Fowler (1944) describes an objective method for determining the frequency of tinnitus by comparison of similar applied frequencies. The procedure begins with the patient listening to a very high tone and then a very low tone and asking which was more like the tinnitus. Then, by continuing this bracketing technique the patient can determine the frequency most closely matching his tinnitus. It was recommended that the external tone be intermittently applied to the non-tinnitus or least involved ear, to avoid confusion in differentiating the test tone from the tinnitus. Fowler further advocated use of intensities which closely approximated that of the tinnitus.

Following development of Fowler's bracketing technique, investigators pursued the study of tinnitus utilizing similar strategies as a means of pitch identification. Reed (1960) relied more heavily on the patient's subjective descriptions of the tinnitus tone; for example, a high-pitched ringing would be assigned a high frequency pure tone to start, using Fowler's bracketing method to approximate the frequency of the tinnitus. At each frequency the patient would be asked to describe in his own terms the difference between his tinnitus and the tone. The intensity of the external tone was also adjusted to closely match that of the tinnitus. The central frequency chosen as the best match with the tinnitus was then varied in both directions until the same frequency 
was selected three times in a row.

Graham and Newby (1962) presented a basic methodology by which the psychophysical measurements of pitch and loudness of tinnitus could be made. Using the 11 frequencies of a pure tone audiometer, an initial pitch match was made. A final discrete frequency pitch match utilizing the bracketing technique described by Fowler (1944) was employed to gain more accurate assessment of pitch of tinnitus. Once this had been determined, a "loudness balance" technique (Fowler, 1941) was used to obtain an approximate measure of the intensity of the tinnitus. This method of loudness balancing called for an objective comparison of the loudness of tinnitus with the tone or noise identified as most closely representing that of the tinnitus. The loudness of the tinnitus would then be recorded as the decibel number, above the threshold for that frequency in the non-test ear (sensation level), necessary to match the loudness of the tinnitus.

From their investigations, Graham and Newby (1962) observed that tinnitus experienced by persons with a conductive hearing loss was comprised of low frequency sounds (120 to $1400 \mathrm{cps}$ ), and suggested that a different physiological mechanism may be involved than that observed in cases of tinnitus associated with other types of hearing loss. Specifically, their observations revealed a significant difference between the range of frequencies noted in the sensorineural and mixed hearing loss groups when compared to the conductive group. The former groups matched the pitch of their tinnitus to a wide frequency range from 155 to $7800 \mathrm{cps}$ for the sensorineural group and from 40 to $6500 \mathrm{cps}$ for the mixed group. Furthermore, the loudness of the tinnitus among 
all three groups was shown to be approximately $5 \mathrm{~dB}$ (SL) for over half of the 73 subjects tested.

In a study patterned after Graham and Newby (1962), Nodar and Graham (1965) investigated the frequency characteristics of tinnitus associated with Meniere's disease. Utilizing a methodology identical to that of Graham and Newby (1962) the authors revealed that the pitch of tinnitus in Meniere's ears was more closely matched to that of tinnitus associated with a conductive hearing loss. Specifically, they noted that the Meniere's group $(N=11)$ had a median pitch match at 320 cps with a range of 90 to $900 \mathrm{cps}$. The conductive group $(N=12)$ had a median frequency of $490 \mathrm{cps}$ with a range of 90 to $1450 \mathrm{cps}$, which was in good agreement with the findings of Graham and Newby (1962) (see Table VI). From these results, Nodar and Graham (1965) concluded that the pitch of tinnitus associated with conductive hearing loss differs significantly from the pitch of tinnitus accompanying sensorineural hearing loss, and the pitch of tinnitus accompanying Meniere's disease was also different than that of any other sensorineural hearing loss. Furthermore, they found that tinnitus in cases of Meniere's was confined to the same low frequencies as the tinnitus associated with a conductive loss, which suggested to them that a similar physiological mechanism was at work in tinnitus accompanying these types of hearing disorders.

More recent attempts to measure the psychophysical parameters of tinnitus have taken many forms and utilized increasingly more sophisticated techniques as our technology and imagination combined to spur on new efforts to solve the mystery of tinnitus. 
TABLE VI

THE PERCENTAGE OF PITCH VIATCHES OF TINNITUS WITHIN FREQUENCY RANGES OF 1000 cPS FOR THE GROUPS WITH MENIERE'S, CONDUCTIVE, AND SENSORINEURAL HEARING LOSS

\begin{tabular}{cccc}
\hline Frequency & $\begin{array}{c}\text { Meniere's } \\
(\mathrm{N}=11)\end{array}$ & $\begin{array}{c}\text { Conductive } \\
(\mathrm{N}=12)\end{array}$ & $\begin{array}{c}\text { Sensorineural } \\
(\mathrm{N}=19)\end{array}$ \\
$8000-$-bove & 0 & 0 & 0.0 \\
$7000-7999$ & 0 & 0 & 21.0 \\
$6000-6999$ & 0 & 0 & 5.3 \\
$5000-5999$ & 0 & 0 & 5.3 \\
$4000-4999$ & 0 & 0 & 10.5 \\
$3000-3999$ & 0 & 0 & 31.6 \\
$2000-2999$ & 0 & 0 & 5.3 \\
$1000-1999$ & 0 & 8.3 & 10.5 \\
$0-999$ & 100 & 91.7 & 10.5 \\
& 100 & & 100
\end{tabular}

(From Nodar and Graham, 1965) 
In an initial attempt to systematically study the effect(s) of deliberately induced tinnitus to the physical characteristics of the inducing stimuli, Loeb and Smith (1967) exposed observers to pure tone and broadband acoustic stimuli ( 90 to $120 \mathrm{~dB}$ ) for a period of 5 minutes. They measured the amount of temporary threshold shift (TTS) induced and had the observers match the pitch of any induced tinnitus by adjusting the frequency of a low level (20 dB SL) pure tone in the opposite ear. They found that the frequency of the tinnitus and frequency of the tone used for the pitch match increased as the frequency of the traumatic stimulus increased. However, they concluded that the reported tinnitus pitch and frequency of maximum loss could not be assumed to coincide for their population. Furthermore, they noted that interobserver variability was considerable (see Table VII).

In a similar study, Atherly et al. (1968) undertook an investigation of tinnitus induced temporarily by noise among subjects exposed in one ear for 5 minutes to $1 / 3$ octave bands of noise centered at either $2,3,4$ or 6000 Hertz $(\mathrm{Hz})$ at $110 \mathrm{~dB}$ SPL. The resulting after-sensation, which they termed noise-induced short-duration tinnitus" (NIST), was matched for pitch and loudness in the non-test ear. They found that the NIST pitch bears a consistent relationship with the point of maximum threshold shift (MTS) and are both related to the center frequency of a $1 / 3$ octave stimulus. They also noted that with each frequency tested the pitch of the NIST is always lower than the MTS (see Table VIII). Perhaps the most iditeresting finding revealed in this investigation was that the difference between the pitch of the NIST and the frequency of the MTS, when expressed as a distance along 


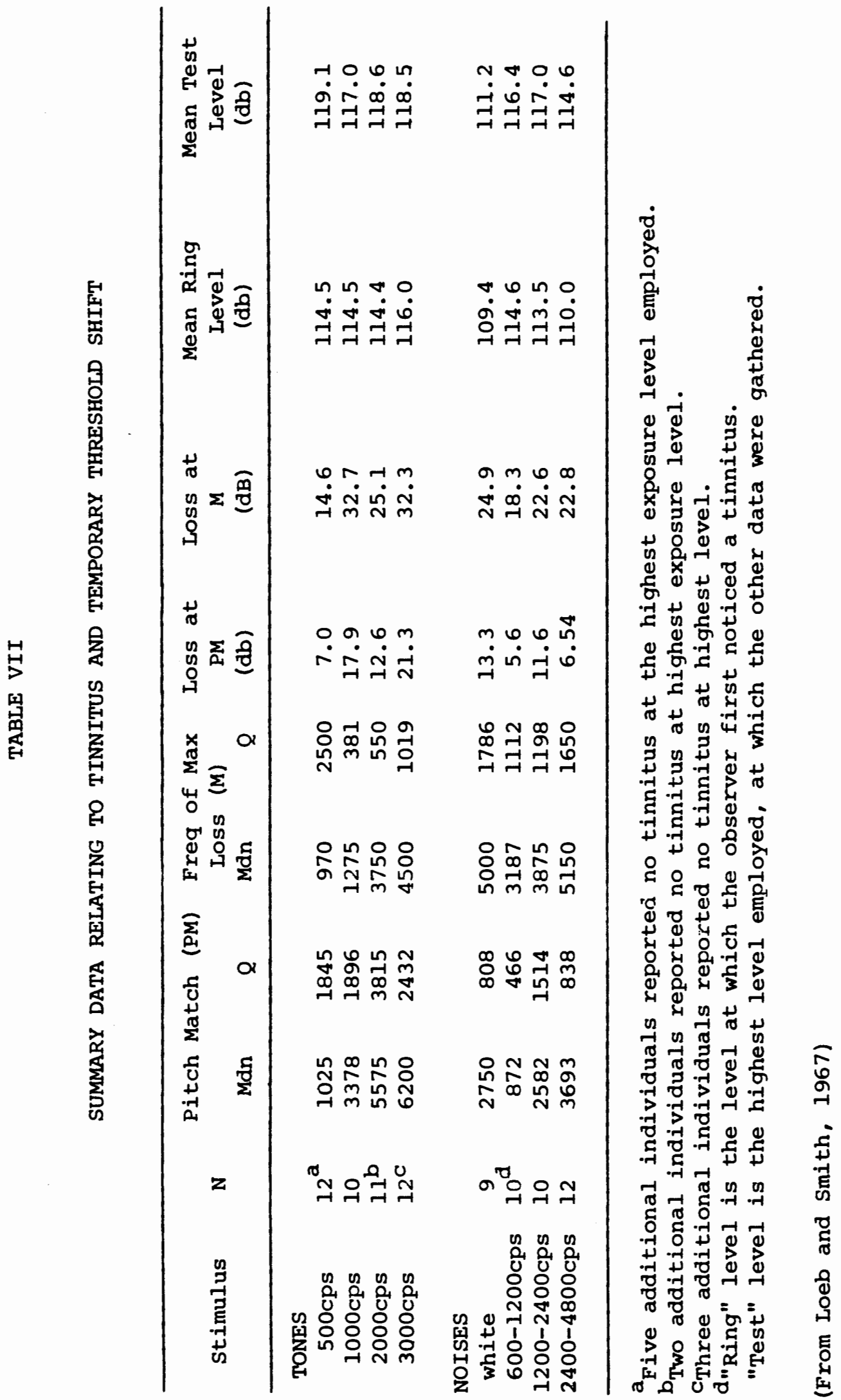


TABLE VIII

EQUIVALENT (MEDIAN) FREQUENCIES OF NOISE-INDUCED SHORTDURATION TINNITUS (NIST) AND FREQUENCIES OF MAXIMUM THRESHOLD SHIFT (MTS) AGAINST STIMULUS CENTER FREQUENCY

\begin{tabular}{cccc}
\hline $\begin{array}{c}\text { Stimulus Center } \\
\text { Frequency } \\
(\mathrm{kHz})\end{array}$ & $\mathrm{N}$ & $\begin{array}{c}\text { NIST Equivalent } \\
\text { Frequency } \\
(\mathrm{kHz})\end{array}$ & $\begin{array}{c}\text { MTS } \\
\text { Frequency } \\
(\mathrm{kHz})\end{array}$ \\
\hline 2 & 7 & 3.0 & 3.5 \\
3 & 25 & 3.9 & 4.5 \\
4 & 7 & 4.9 & 5.6 \\
6 & 11 & 6.5 & 8.0
\end{tabular}

(From Atherly et al., 1968) 
the basilar membrane, was independent of stimulus frequency and was constant at about $1.3 \mathrm{~mm}$, which corresponds to one critical bandwidth.

A short time later, Feldmann (1971) investigated the maskability of tinnitus using broad-band and narrow-band noise and pure tones. Among a group of approximately 200 subjects, he employed one of the above masking stimuli, prescrted at octave intervals, and obtained five types of patterns classified and associated with different etiologies based on the configurations produced by the masking levels. Figure 8 shows the various patterns revealed in Feldmann's work. He labeled Type I "Convergence type", which he found in $34 \%$ of his subjects describing their tinnitus as high pitched. It was present in cases of noise-induced hearing loss and exhibited a predominantly high frequency loss. Type II was labeled "Divergence Type." It was observed only rarely (3\%) and Feldmann could not assign a specific pathology to this type. Type III, "Congruence type," was noted in $32 \%$ of the cases and appeared to be associated with flat audiograms occurring most of ten in cases of Meniere's disease, sudden deafness, and otosclerosis. Type IV, "Distance typen, was present in cases of various pathologies of the middle and inner ear and was seen in $20 \%$ of the cases. Type $V$ was designated "Resistance type" because the tinnitus could not be masked at any stimulus intensity. It was observed in $11 \%$ of those tested and mostly in severe cases of sensorineural hearing loss or complete deafness.

From these masking studies, Feldmann drew two distinct conclusions. First, the spontaneous activity of the hair cells of nerve fibers responsible for the tinnitus, derived an entirely different masking 

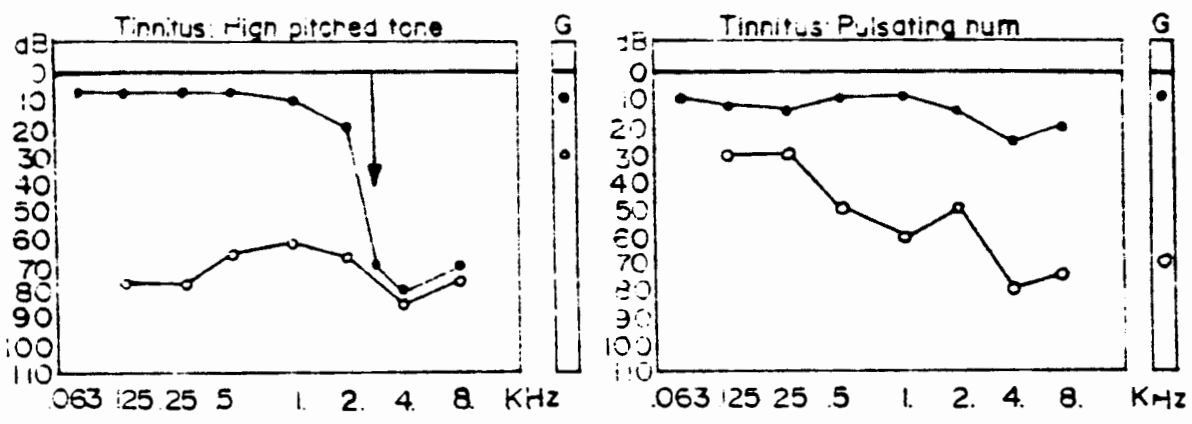

1 Convergerce iyce

II Divergerce type
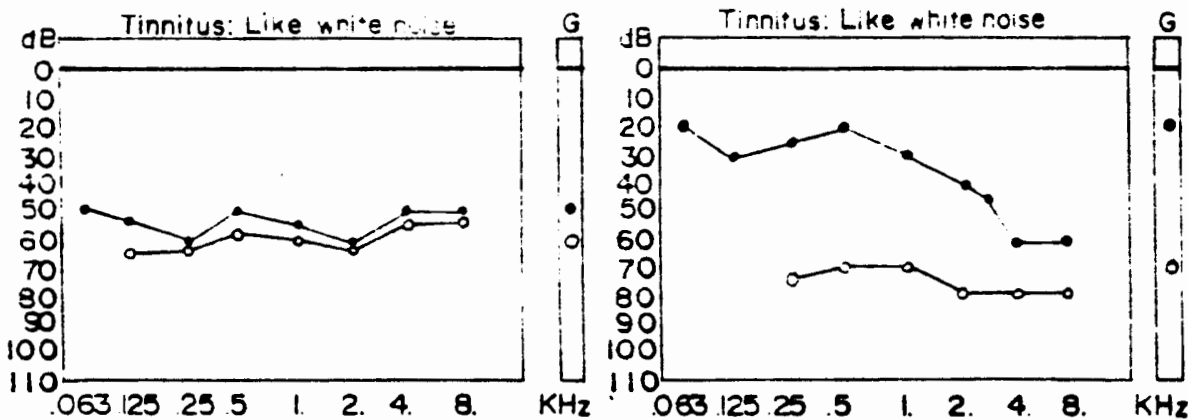

III Congruence :ype

IV Cistance type

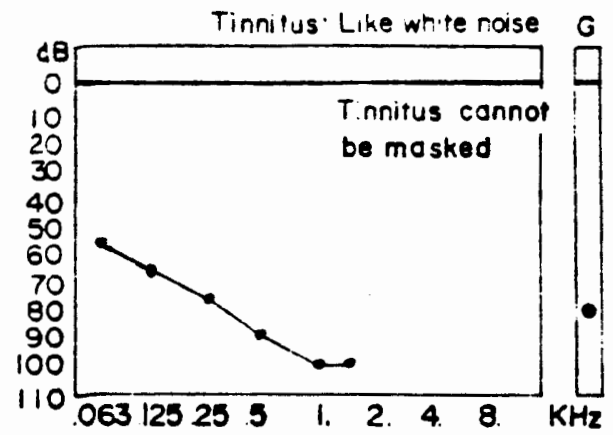

$\checkmark$ Persistence iypo

Figure 8. Different types of masking curves of tinnitus (From Feldman, 1971).

$\leadsto$ Pure tone air conduction thresholds

Narrow Band Noise (1/3 octave)
- Threshold of white noise

- White noise 
pattern than that generated by the external stimulus evoking the same subjective sensation. Second, he felt that the masking pattern obtained in the tinnitus subjects could not be explained on the basis of some physical interaction along the basilar membrane, but rather may be more adequately interpreted as a form of neural inhibition. He illustrated this point by noting that during threshold audiometry or presentation of narrow band noises to the affected ear, the tinnitus ceased as threshold was reached, regardless of frequency. He speculated that this might be due to a lateral inhibition which spreads from the region activated by the external stimulus and reduces or covers the pathological spontaneous activity in all other regions.

More recently, the use of masking signals (generally, bands of noise) have been employed to plot masking curves of tinnitus in an effort to locate an optimum frequency range and intensity level which would effectively mask a patient's tinnitus (Vernon, 1975; Vernon et al., 1977; Vernon \& Schleuning, 1978) (see Figure 9). The development of current masking techniques by Vernon and his associates at the Kresge Hearing Research Laboratory, which successfully mask the tinnitus, are based on several parameters, the first of which is determining what they call the minimum masking level (MM). This is defined as the "masking sound that effectively masks the tinnitus at the lowest sensation level" (Vernon \& Meikle, 1981, p. 243). They state that the lower the sensation level of effective masking stimuli, the greater the probability of the patient accepting and gaining relief from the externally produced sound. Stated differently, the acceptability of tinnitus masking is inversely proportional to its loudness. 

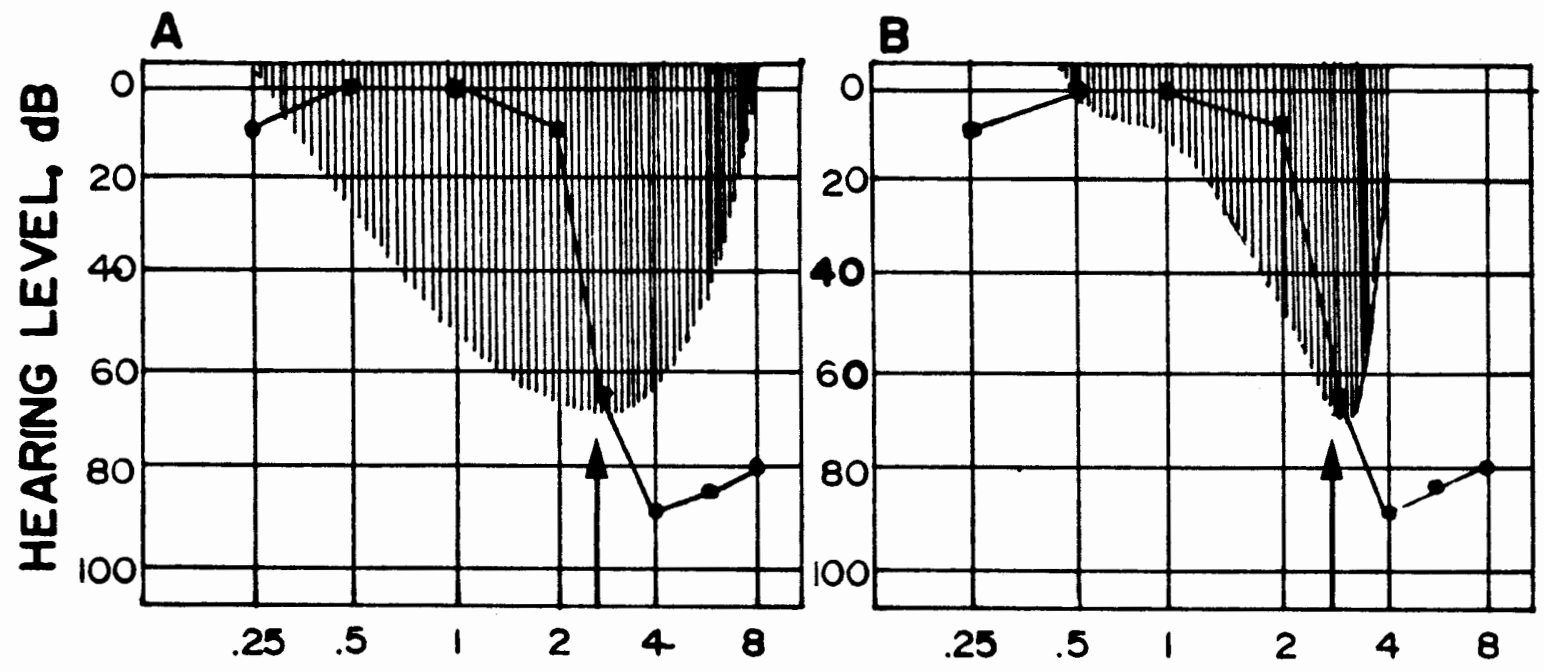

\section{FREQUENCY, KHZ}

Figure 9. Acoustic characteristics of masking in relation to the individual's hearing capabilities. Audiometric data for a tinnitus patient with abrupt high-frequency hearing loss are shown here with tinnitus masking bands schematized by shaded areas. The same audiogram is plotted in (A) and (B). Tinnitus frequency $\left(F_{T}\right)$ is indicated by arrow. (A): Broad-band masking noise which is easily available but which would be unacceptable to this type of patient, as too much sound energy is provided at the lower frequencies, where hearing is normal. (B): A hypothetical masking band which is more restricted to the region of the patient's tinnitus but which is difficult or impossible to achieve with present masking equipment. (From Vernon and Meikle, 1981) 
The procedure used to determine ML is described briefly by Vernon and Meikle (1981):

- We present a series of 4-6 different tones or noise bands, arranged in order throughout the patient's usable hearing range, and we record the minimum sensation level required at each frequency to provide complete masking of the tinnitus. This minimum level is established using an ascending series of sound levels separated by intervals of 2 dB. . In this way we determine the MML at each frequency; from this array of Mll s we can then determine whether there is a trend and whether masking is more effective within a particular region of the frequency scale. If we take $F_{T}$ to represent the frequency most closely matching the pitch of the tinnitus, in most cases (but not all) the lowest MML are obtained in the region of $F_{T}$.

In their efforts to determine the most appropriate method for evaluating the loudness of tinnitus, the above authors offer the following current method used at the Tinnitus Clinic:

Our present method for measuring the loudness of tinnitus extends Fowler's (1942) method to a wider frequency range. After $F_{T}$ has been determined, the sound level at $F_{T}$ is increased in small increments (1-2 dB) until it just matches the loudness of the patient's tinnitus, and that level is recorded in $d B$ sensation level. A similar ascending series of sound levels is used to obtain loudness matches at 4-6 frequencies distributed throughout the patient's usable range of hearing.

From their investigations dealing with methods of tinnitus evaluation, Vernon and Meikle (1981) demonstrate that the shape of the curve relating loudness to frequency is different for each patient (see Figure 10). Whether or not the shape of the loudness matching curve can be used to predict any dependence of MLs on frequency is not known, but is alluded to in their clinical observations.

Along with their methods for evaluating pitch and loudness of tinnitus, Vernon and Meikle (1981) note two precautions they feel are important in determining $F_{T}$ and its accompanying loudness level. First, 

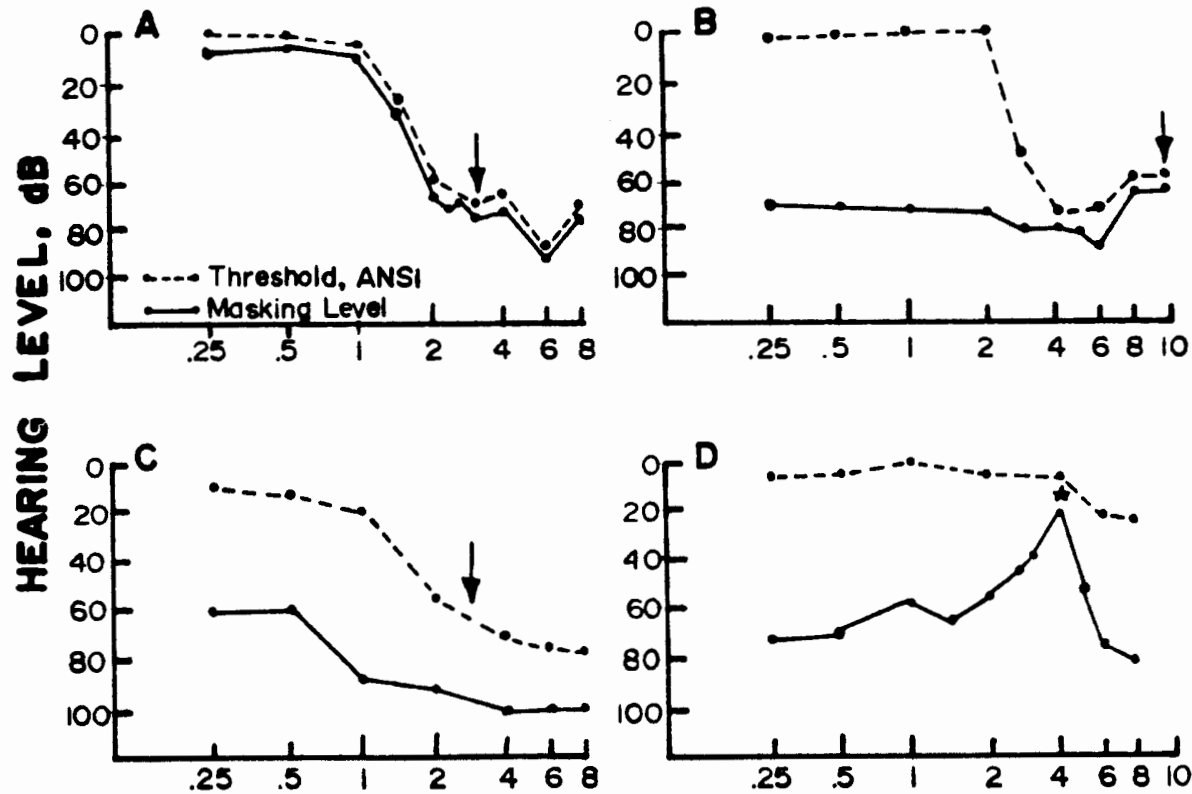

FREQUENCY, KHZ

Figure 10. Masking of tinnitus. Masking of tinnitus by pure tones was studied in 32 cases of tonal tinnitus. Three major types of masking function were encountered, as illustrated in $A, B$, and $C$, using data from one representative case in each group. For comparison, (D) shows standard type of tone-on-tone masking function obtained for $4 \mathrm{kHz}$ tone in one normal subject. (All graphs, unpublished data of C. Mitchell). Tinnitus masking curves described as follows: A, 18 of cases, masker sensation levels (SL) very low, and independent of frequency; $B, 528$ of cases, masker SL's decreased as test frequency approached $\mathrm{F}_{\mathrm{T}} ; \mathrm{C}, 228$ of cases, masker SL's relatively high and independent of frequency; (remaining 88 of cases were mixed and did not fit any of the above categories.) As (D) shows, tone-ontone masking exhibited the expected sharp peak (SI's declined sharply) as masker frequency approached $4 \mathrm{kHz}$, with masker effectiveness falling away rapidly on either side of that point. All three types of tinnitus masking function show considerably greater spread of masking effects around $F_{T}$, ranging from $1-2$ octaves $(B, C)$ to more than three octaves (A). ANSI, American National Standards Institute. (From Vernon \& Meikle, 1981) 
they stress that the loudness of the comparison tone (usually presented contralaterally unless hearing in that ear is too poor) must be properly balanced with that of the tinnitus at each frequency tested. This allows the patient to make more accurate pitch comparisons with the tinnitus at that frequency. The second precaution is to test for what they refer to as the "octave confusion." They have noted that in some cases the first frequency match selected by the patient of ten proves to be in error by one and sometimes two octaves. Therefore, frequencies at octave multiples of the first identified pitch must be presented to avoid this error in pitch judgment.

The final measure employed in evaluating tinnitus involves the phenomenon of residual inhibition (RI). It is described by Vernon (1977), as a persistence of the masking effect upon tinnitus after the masking has ceased (see Figure 11). Although the term was not coined until recently (Vernon, 1977), it was alluded to in earlier studies by Spaulding (1903), Jones and Knudsen (1928), Josephson (1931), and more recently by Feldwann (1971). The latter author suggested that the cessation of the tinnitus after exposure to an external tone for a specified time was due to some form of neural inhibition.

Vernon (1981) has noted that residual inhibition can come in a variety of forms. The method used at the Tinnitus Clinic to measure RI is a simple procedure. The tone which best matches the tinnitus is adjusted to $M M L+10 \mathrm{~dB}$. The test tone is presented for one minute, after which time the patient is asked to describe his tinnitus. In about $35 \%$ of the cases reported by Vernon and his colleagues at the Tinnitus Clinic, the tinnitus is absent or RI is complete (CRI) for 


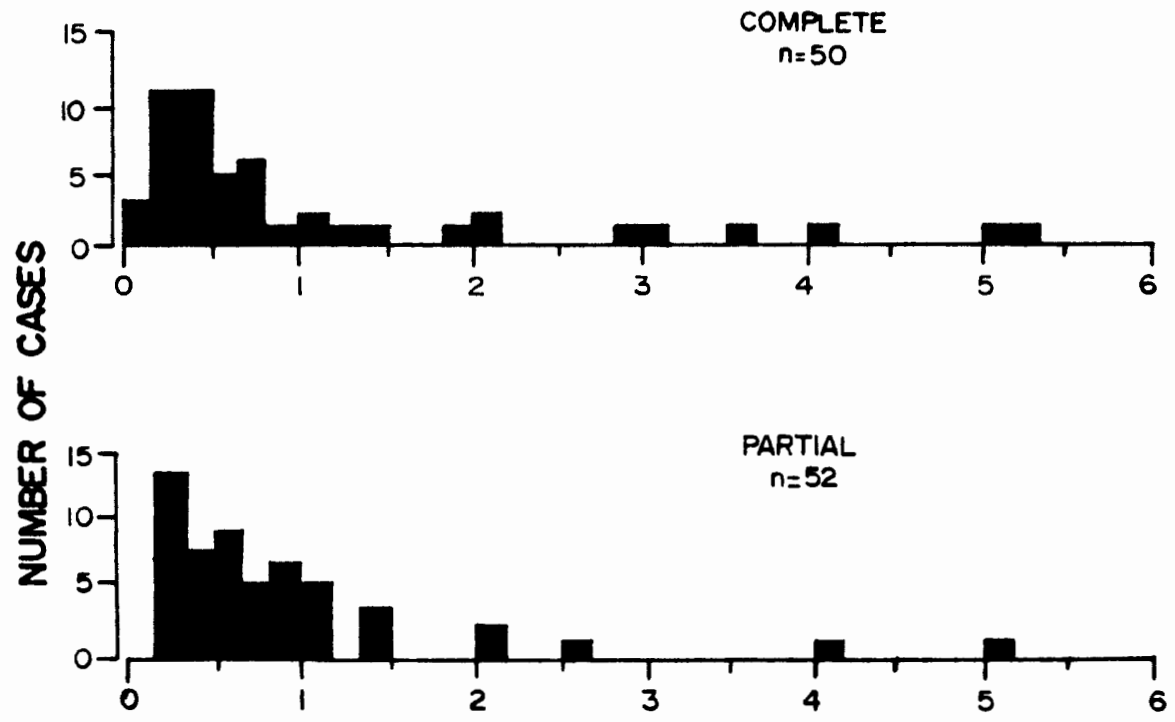

TIME, $\min$.

Figure 11. Duration of residual inhibition following one minute of masking. Graphs were compiled from records of 63 patients chosen quasi-randomly from the Tinnitus Clinic files and exhibiting a positive response when tested for residual inhibition. Complete residual inhibition (CRI) was experienced by 50 of these patients (upper graph), followed by partial residual inhibition (PRI) in 39 of them. An additional 13 patients experienced PRI without first experiencing CRI, bringing the total in the lower graph to $39+13=52$. Each small division on the horizontal scale represents a bin width of 10 seconds, and contains all cases in which residual inhibition lasted longer than the left hand-boundary, but ended at or before the right-hand boundary. For example, in the upper graph there were three cases in which CRI lasted between $1-10$ seconds (first bin) and 11 cases in which residual inhibition lasted between 11-20 seconds (second bin). (From Vernon and Meikle, 1981) 
about 25 to 35 seconds, and in some cases longer. In other instances (43\%), they report it is only partial (PRI) before returning to its normal level. In some extreme cases they report CRI lasting for several hours after one minute of masking. They have also shown that extending the masking time affects both the magnitude and duration of RI (Vernon \& Meikle, 1981) (see Figure 11).

Based on these results, Vernon and Meikle (1981) suggest that the most effective way to produce RI is to provide masking that most closely resembles the tinnitus. Their studies suggest that the most acceptable masking sound is not necessarily the most effective, particularly where RI is concerned. Furthermore, they note that pure tone masking of tinnitus is generally more effective than masking with narrow band noise centered at the same frequency, yet less acceptable. In addition, they have observed, as did Feldmann (1971), that some cases of bilateral tinnitus can be masked by application of unilateral masking. They also note that in fewer than $10 \%$ of these cases, RI is produced in the contralateral ear. A final observation involves a situation in which masking of tinnitus is complete, yet fails to produce RI. This has been observed to occur in patients who obtain complete masking of tinnitus through amplification. Vernon \& Meikle (1981) feel that the use of hearing aids in masking tinnitus is effective when ambient noise is at a level that reduces or eliminates the tinnitus, yet does not interfere with hearing. Because of the differences between RI and masking, they tend to support the hypothesis that RI depends more on selective application of acoustical stimuli than does masking. 
From such observations, Vernon and Meikle (1981) suggest that RI may represent some form of neural plasticity that is capable of producing such long-duration changes after such short-duration stimulation. However, they agree that there is need for further investigation designed to evaluate various masking stimuli and their ability to produce RI.

In a study conducted by Pennar et al. (1981), which compares the temporal course of masking of tinnitus with external tones, it was revealed that for subjects with noise-induced hearing loss the noise intensity required to mask their tinnitus increased markedly during exposure to broadband noise (see Figure 12a, b, c). In contrast, noise intensity which masked an external tone remained nearly constant. From these findings, they inferred that since the tinnitus became audible in noise which previously masked it, the assumption could be made that the kinds of neural activity which underlie tinnitus do not exhibit fatigue. Therefore, they concluded that the absence of tinnitus fatigue implied either more central locus, beyond the auditory periphery in the brainstem, or that tinnitus involves N. VIII activity which does not fatigue.

Vernon (1981) also feels that factors underlying tinnitus do not include adaptation, fatigue or temporary threshold shift (TTS). He suggested that these Ideas were Imprecise because of the time elements involved. He offered as an example a case where RI lasted for hours after only a short period of masking at 20 to $30 \mathrm{~dB}$ SL. He also reported that Sandlin has personnally communicated to him that during RI no change in hearing threshold is observed for frequencies corresponding 

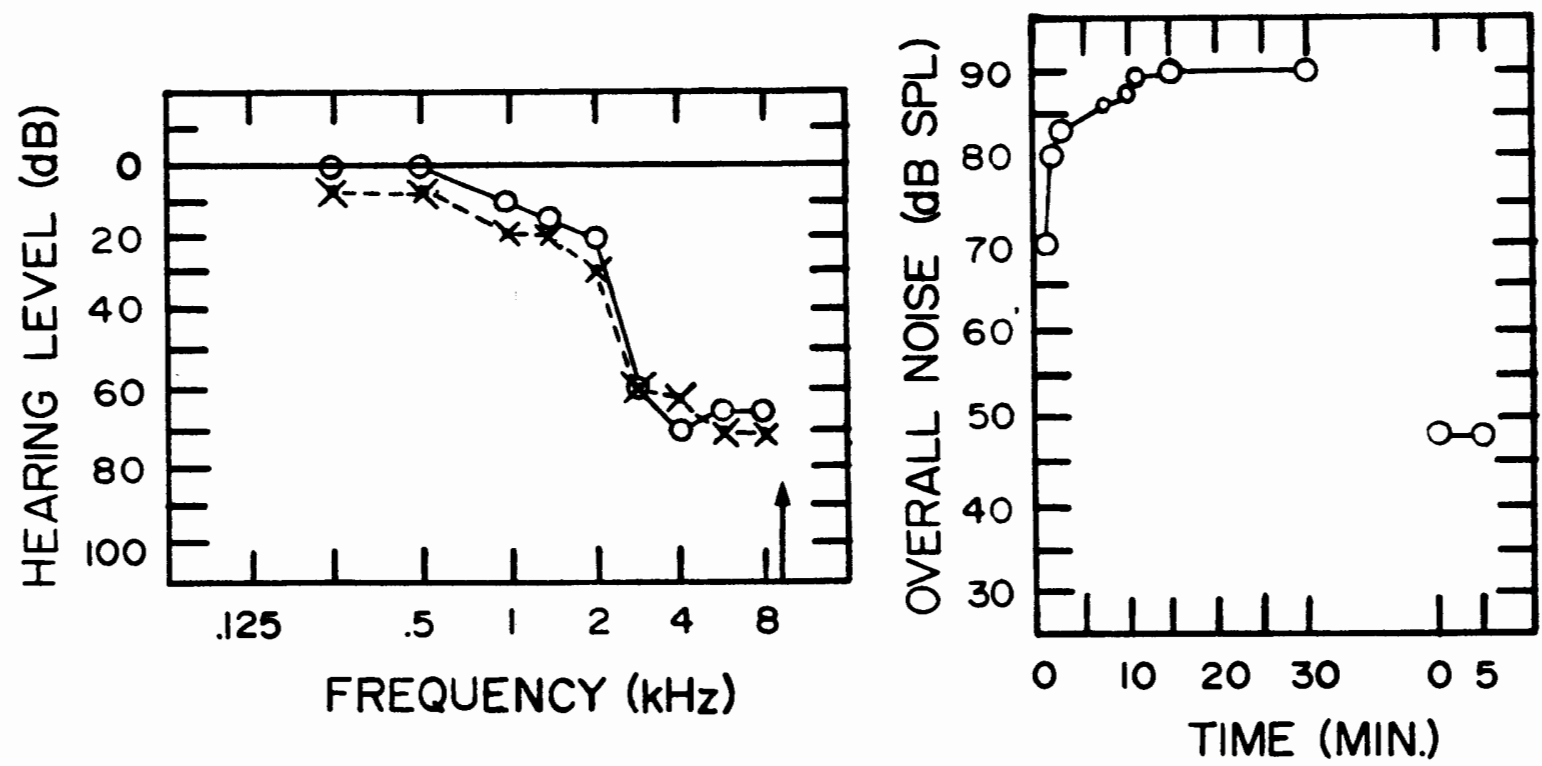

Figure 12a. The audiogram and the temporal course of masking for internal and external tones for subject $A$. The acoustic reflex occurred at $90 \mathrm{~dB}$ overall in the right and $95 \mathrm{~dB}$ overall in the left ear in the presence of a broad-band noise. The audiogram on the left displays the threshold for the right (the circles) and the left (the $x^{\prime} s$ ) ears. The arrow marks the frequency of the tone said to match the tinnitus in quiet. The graph on the right displays the noise required to mask the internal tone (the left) and the external tone (the right) as a function of time since the noise onset. (From Pennar et al., 1981) 

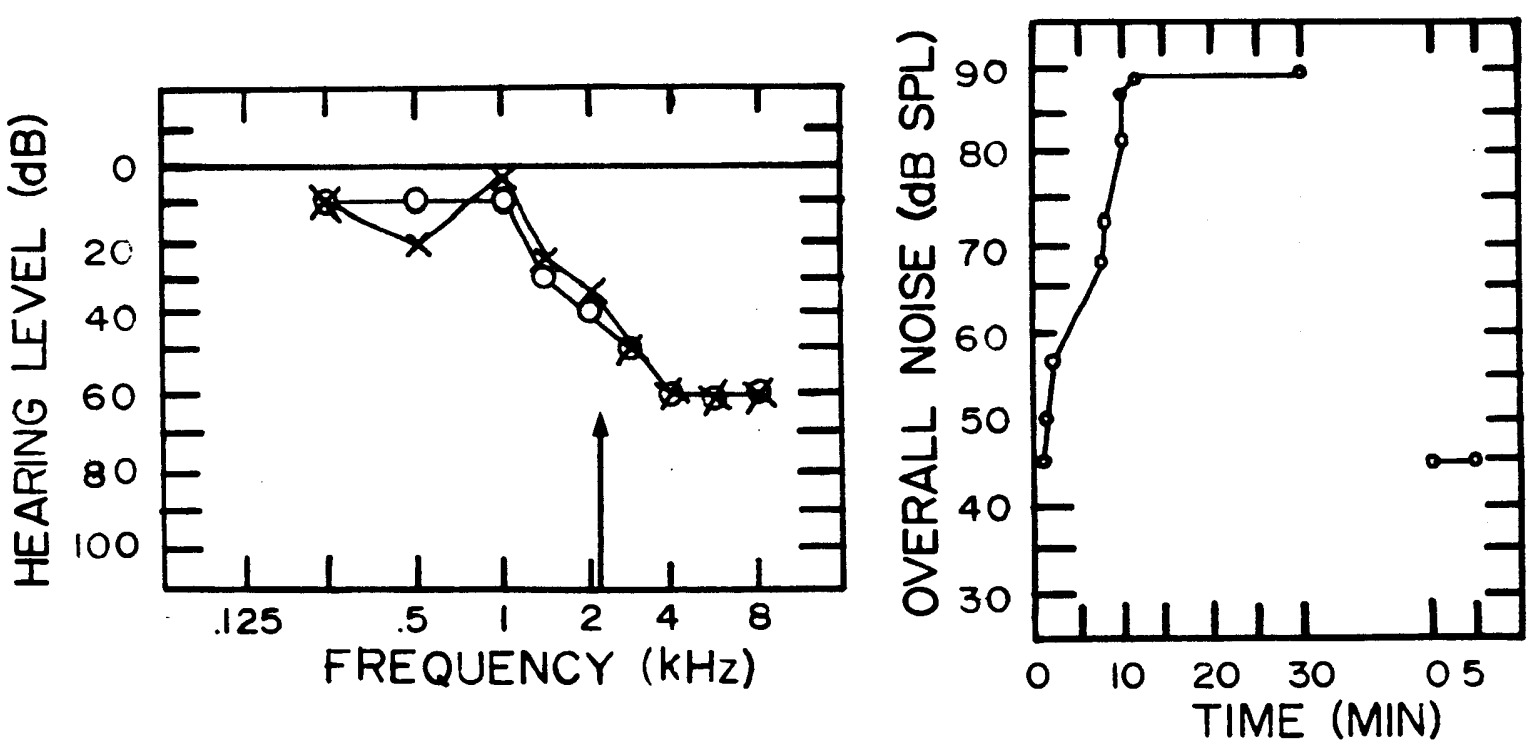

Figure 12b. Audiogram and temporal masking data for subject $B$. The acoustic reflex for this subject was activated with $85 \mathrm{~dB}$ overall in the right and $80 \mathrm{~dB}$ overall in the left ear. (From Pennar et al., 1981)
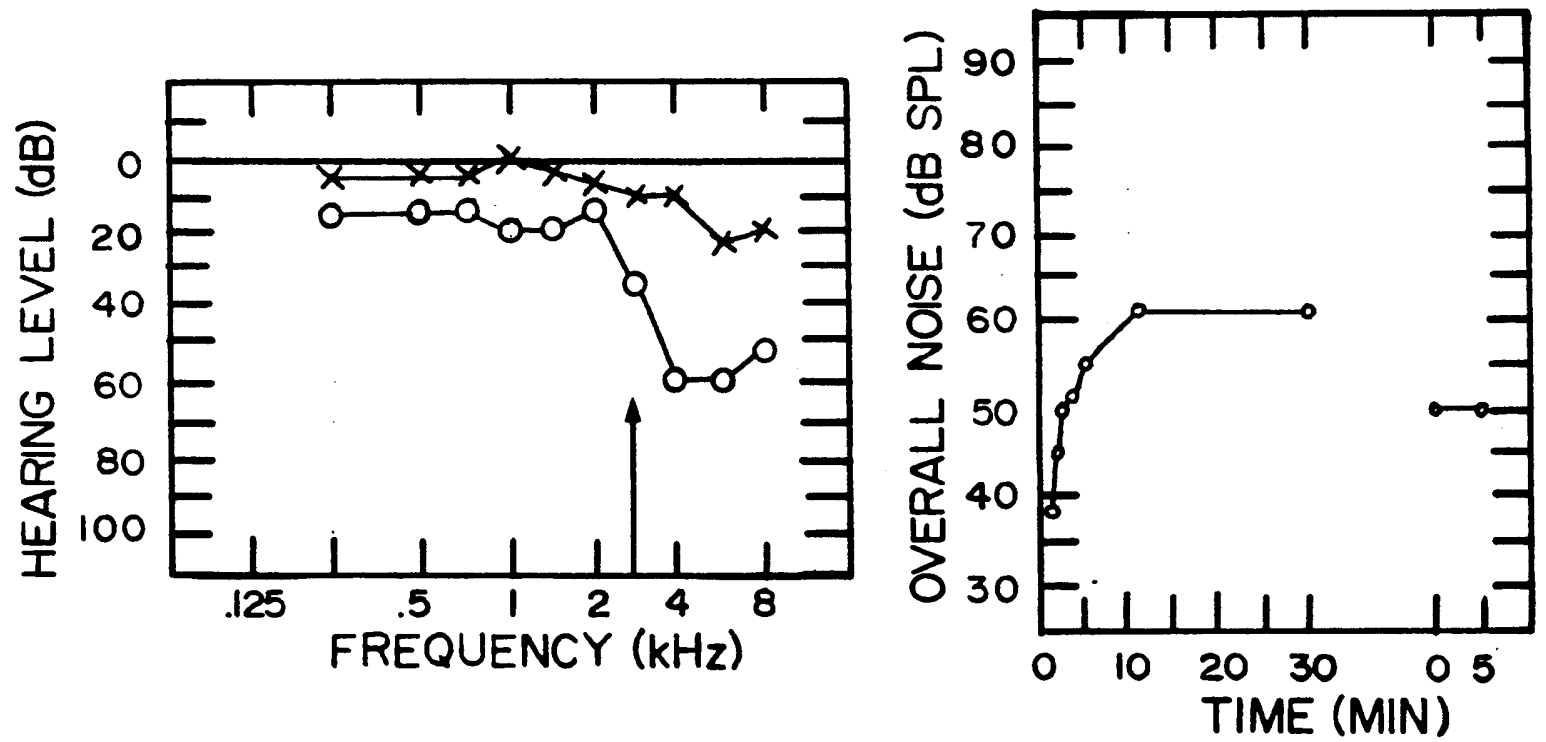

Figure 12c. Audiogram and temporal masking data for subject $C$. The acoustic reflex threshold was $90 \mathrm{~dB}$ SPL overall in each ear. C reported that the tinnitus was monaural. The level of noise presented in the contralateral ear increased as shown. (From Pennar et al., 1981) 
to that of the tinnitus, thus ruling out any possibility of TTS involvement. However, it is interesting to note that in some cases of RI the gradual return of the tinnitus is negatively accelerated (Vernon, 1981) and that both RI and adaptation appear to modify the sensitivity of the sensory system as a result of continuous stimulation at a constant intensity level.

other more recent attempts to measure the parameters of tinnitus have utilized highly sophisticated techniques in an effort to solve the mystery of tinnitus. Kemp (1978), investigating stimulated acoustic emissions from within the human auditory system, has devised a technique utilizing signal averaging of the response of the closed external auditory meatus to acoustic impulses (click stimuli) near the threshold of audibility. The slowly decaying response (echo) recorded via a probe microphone sealed in the ear canal, was present in the majority of, if not all, normal human ears, but not in ears with cochlear deafness. The mechanism responsible for this cochlear reflection was thought to be the result of a stimulated release of coherent mechanical energy onto the basilar membrane locally modifying wave propagation parameters. As a result, at very low levels the critically set mechanism might "over-react" at some frequencies, with the recoil producing a retrograde wave on the basilar membrane eventually appearing in the external auditory meatus as a "cochlear echo." Kemp (1978) further speculated that involvement of the outer hair cells in the generation of this mechanical energy may be implicated.

The significance of Kemp's (1978) discovery and its relationship to tinnitus was revealed in the investigations of Wilson (1980). He 
hypothesized that the compressive nonlinearity of the response (acoustic echo) provided evidence for "active" processing and frequency tuning. Furthermore, he observed that tinnitus could be induced by inverting body posture and that properties described as beating were objectively observed in 10 ears ( 30 ears not producing either observation). He concluded that these findings, combined with Kemp's (1978) demonstration of the peripheral auditory system's ability to generate audio frequency sounds lent support to his view that acoustic echoes, threshold fine-structure, and tinnitus are interrelated and originate within the cochlea. However, a personal communication with Meikle (1983) has clarified Kemp's interpretation of the acoustic echo and its generating source as it relates to tinnitus. She states that upon speaking with Kemp, in person, it was his belief that much research must be accomplished before any correlation between the stimulated acoustic echo, its generating source and tinnitus can be made.

Animal models for examining tinnitus have involved study of the afferent pathways in the brain. One such study by Sasaki et al. (1980) involved measurements of glucose uptake in the auditory pathway after surgical section of the cochlear nerve. Their results indicate that increased glucose uptake at higher auditory centers was related to increased neuronal activity after cochlear ablation and may represent an analog of the tinnitus phenomenon in man.

The use of reaction time measurements in tinnitus patients has been investigated by Goodwin and Johnson (1980). Three frequency regions tested in their study were: the tinnitus frequency (with abnormal thresholds), nontinnitus frequency (thresholds similar to tinnitus 
frequencies), and nontinnitus frequency (threshold within normal limits). Using an elaborate switching and timing device, reaction times were determined for threshold response for these frequencies. Results of this investigation suggest that measurement of reaction time to auditory stimuli provides an objective measure of tinnitus, as well as determining the presence of recruitment, and a possible technique for mapping areas of tinnitus.

In an unpublished study by Burns (1982), the variability in measuring the pitch and loudness of subjective tinnitus was investigated. After several practice sessions to familiarize themselves with the method-of-adjustment paradigm for pitch and loudness matching, eight subjects were asked to listen to acoustic stimuli consisting of three pure tones $(100,500$, and $1,000 \mathrm{~Hz})$ and three narrow bands of noise each 100,500 and $1,000 \mathrm{~Hz}$ wide, and match them with their tinnitus. After tinnitus matching had been achieved, a similar procedure was used in order to try to mask their their tinnitus using a $1,000 \mathrm{~Hz}$ band of noise. His results indicate that for all subjects tested, the variability in pitch and loudness matching of tinnitus is greater than that for pitch and loudness matching of objective stimuli approximating the frequency and intensity of their tinnitus. He noted that his findings agreed well with previous studies (Fowler, 1944; Feldman, 1971). From his results, Burns (1982) concluded that the large variability in tinnitus matching and the lack of normal frequency-specific masking of tinnitus in his subjects was the result of more central mechanism and not primarily the results of any peripheral impairment. 
Contemporary Methods of Treatment

From these early investigations into the origin and psychoacoustical parameters of tinnitus, the next logical topic in our discussion of the literature involves modern treatment. Unfortunately, modern medicine has little to offer in the way of a curative agent for tinnitus at present. However, many investigators are currently studying the effects of drugs and other therapeutic measures in an effort to reduce or eliminate the distressing effects of tinnitus.

of the three major methods of treatment now available, masking, biofeedback and medical, Shea and Emmett (1981) believe that medical treatment has the most promise. The success of any treatment rests on accurate diagnosis of the site of lesion and etiology of the complaint of tinnitus, which should be carried out through the combined efforts of the audiologist and otologist (Shulman, 1981).

Following complete cochleo-vestibular evaluation of all patients complaining of hearing loss, vertigo or tinnitus, alone or in combination, Shulman (1981) reported on clinical experience gained from the use of antihistamine/vasodilator medication. Results from his clinical findings revealed that 38 of 178 patients (22\%) benefited from use of vasodilator drugs.

Shea and Emmett (1981) have also reported on the use of intravenous lidocaine and oral anticonvulsants previously used by Melding et al. (1978) at the Pain Clinic in Auckland, New Zealand, for diagnosis of pain. It was Melding's group which first discovered that these drugs provided excellent (56\%) or partial (24\%) relief from tinnitus among 100 patients studied. Shea and Emmett (1981), using swaller doses of these 
types of drugs have also achieved similar results with $78 \%$ of their group attaining partial relief and $4 \%$ achieving total relief from tinnitus.

Because the concept of sensory epilepsy has been postulated in the pathophysiology of central pain and tinnitus, the use of oral anticonvulsants has provided an area of investigation which holds promise as an effective medical treatment in cases of tinnitus. Until such treatment becomes available, Shea and Emmett (1981) offer a synergistic approach utilizing chemical, masking and/or biofeedback for relief from tinnitus.

Emmett and Shea (1981) have also reported on the use of an lodinated dye (Hyaque) used in radiologic studies. Its initial value in relieving tinnitus was discovered by accident, as an added benefit from restoration of hearing in cases of sudden idiopathic hearing loss. Fifteen patients seen by Emmett and Shea had complete recovery from hearing loss. Of these, $50 \%$ had no change in their tinnitus, while $25 \%$ had no tinnitus following recovery of hearing, and the remaining $25 \%$ had less severe tinnitus.

Cazals et al. (1978) reported the cancellation of tinnitus during round window or promontory electrical stimulation with a positive current. The effect was noted only ipsilaterally and was presumed to have an inhibiting effect on abnormal activity in the N. VIII, whether hair cells were functioning or not.

Ambrosino (1981) feels that tinnitus is a form of stress, compounded by the fact that it is most often associated with a hearing loss. Marlowe (1973) has reported some success with hypnosis by drawing 
attention to the fact that tinnitus is a stress that can affect the muscular tension and vasoconstriction. Grossan (1976) reported success with biofeedback, as did House (1977), who reported 80-90\% improvement from among a group of 41 patients. He states that almost all of the subjects in his study gained some benefit from the treatment. However, he felt that much of their anxiety and depression was misplaced on their tinnitus, resulting in subjectively louder perception of tinnitus. He concluded that biofeedback is helpful in reducing tinnitus in that it allows the patient to become more aware of their emotions, thereby reducing the subjective loudness, enabling him to better understand and cope with it.

Since the earlier finding of Saltzman and Ersner (1947) that a hearing aid was beneficial in relieving tinnitus in some patients, researchers have continued in their efforts to provide adequate amplification, and more recently, masking of tinnitus.

As a result of the efforts of Vernon and his colleagues, a Tinnitus Clinic was established at the Kresge Hearing Research Laboratory (1977). It was by chance that Vernon discovered that an external sound of a certain quality would cover or mask the internally generated sound of tinnitus. Since then, much research has culminated in the development of the tinnitus masker. This is a device which introduces a masking sound into the ear and is housed either in a post-auricular or all-in-the-ear hearing aid case. The first maskers developed contained sound energy encompassing only a very restricted band of 300 to $3000 \mathrm{~Hz}$. This proved to be ineffective as masking stimulus for those with higher-pitched tinnitus (Vernon, 1976; Vernon \& Meikle, 1981). 
As technology advanced in the hearing aid industry, the ability to effectively aid and/or mask those with high-frequency tinnitus became more accessible. Figure 13 shows the representative response ranges of maskers as their development has progressed. The continued upward spread of frequency ranges avallable for masking has resulted in the successful masking of a patient with tinnitus reported at $17,000 \mathrm{~Hz}$ (Vernon \& Meikle, 1981).

The masking program at the Tinnitus Clinic utilizes three types of masking instruments. Following medical examination to rule out physical or pathological problems which would exclude them from the masking program, an audiological and tinnitus evaluation are performed (Schleuning et al., 1980). The three types of masking which provide relief from tinnitus are derived from the application of a hearing aid, a tinnitus masker (wideband or high-frequency emphasis), or a tinnitus instrument, which is a combination aid and masker (Vernon et al., 1977). A follow-up study of two groups of tinnitus patients seen in the Tinnitus Clinic from 1976-1979 was conducted in an attempt to evaluate the success of the masking program. Of 598 patients receiving a questionnaire, 493 responses were received. The patients were divided into two groups, the first containing responses from those involved in the program from 1976 through 1978, the second containing responses from those participating in 1979. Results from both groups are displayed in Tables IX and $X$, respectively. In the earlier stages of the program it can be seen that use of maskers was not very successful (61 of 204 recommendations, or $30 \%$ currently wearing unit). However, this has been attributed to the poor electroacoustical characteristics inherent in the 


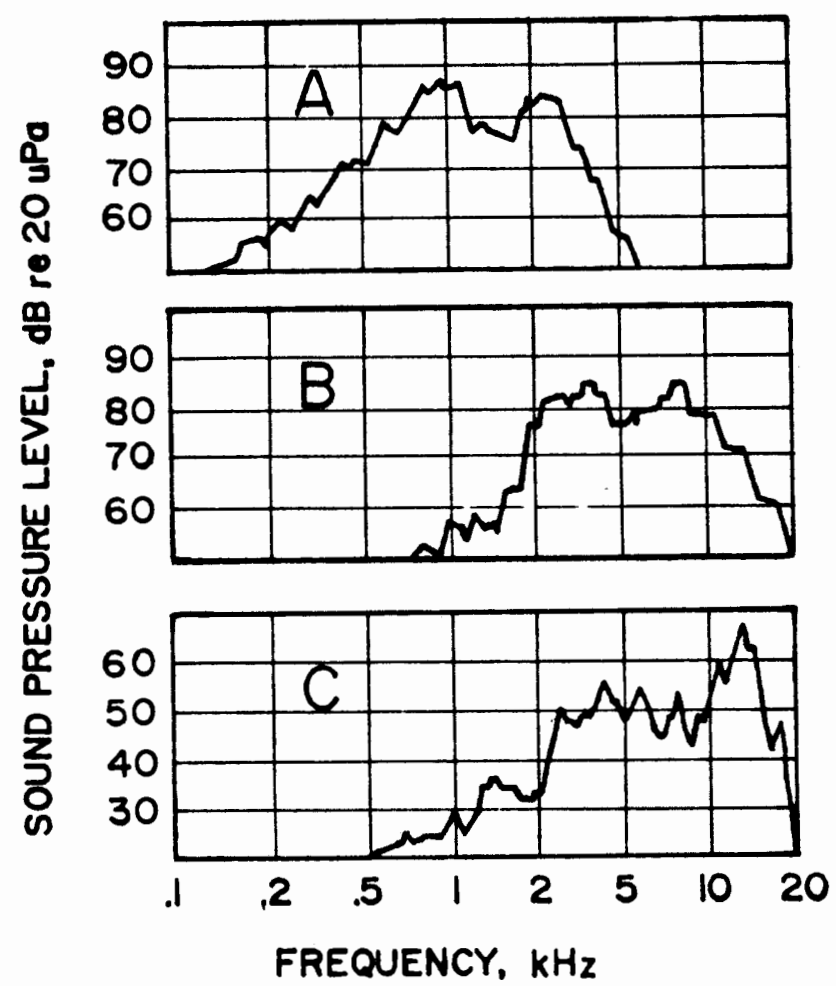

Figure 13. Frequency response of representative tinnitus maskers. All curves were obtained using KEMAR (Knowles Electronic Mannikin for Acoustic Research); gain control of each masker was set at minimum output level. Note vertical scale in (C) differs from that in (A) and (B). (A): First tinnitus masker developed, with relatively broad band centered around 1 to $2 \mathrm{KHz}$. (B): A more recent masker, showing higher-frequency emphasis, with noise band centered around $5 \mathrm{kHz}$. (C): One of the most recent maskers, with pronounced high-frequency emphasis and very little energy below $3 \mathrm{kHz}$.

(From Vernon and Meikle, 1981) 


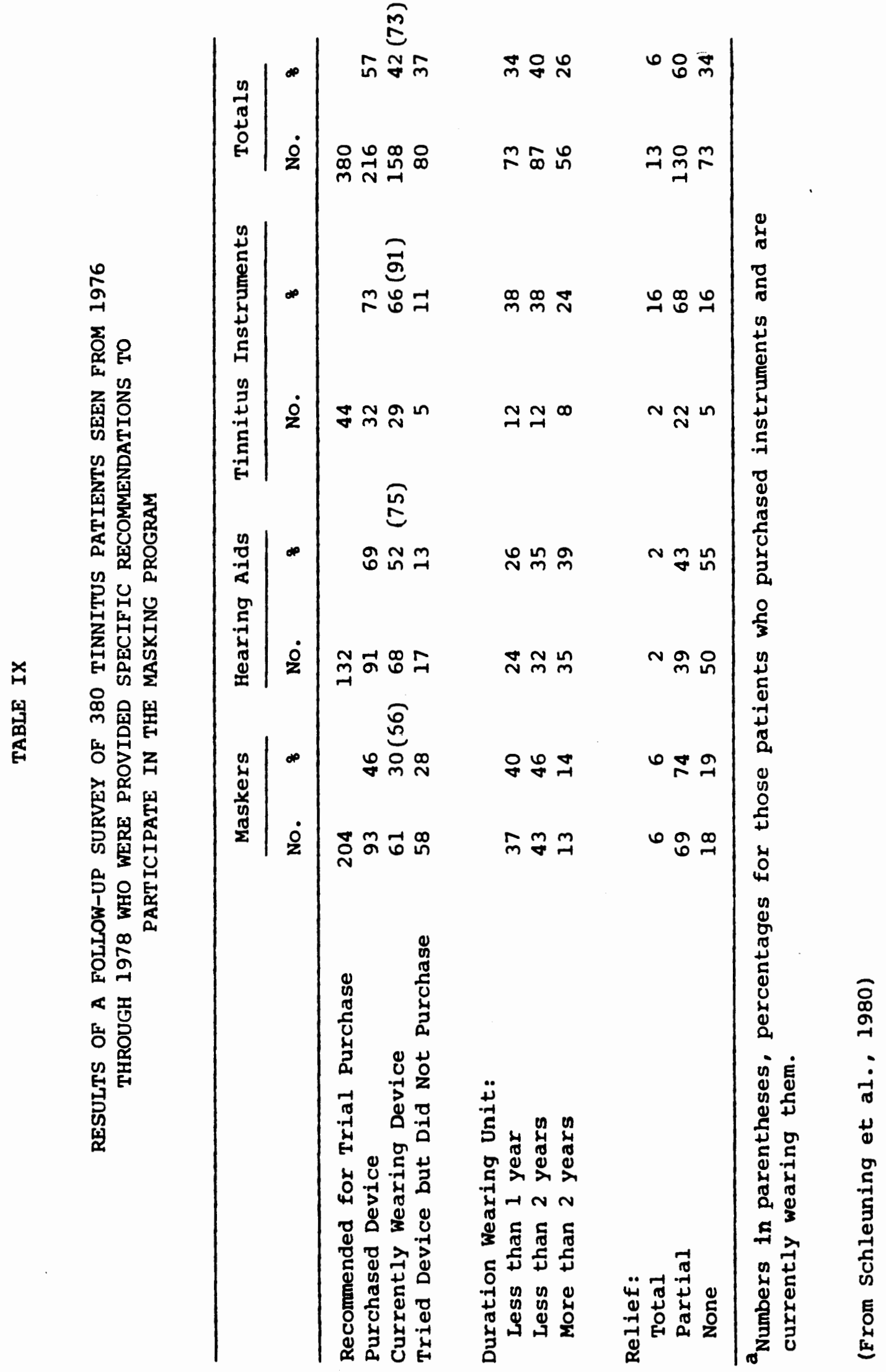




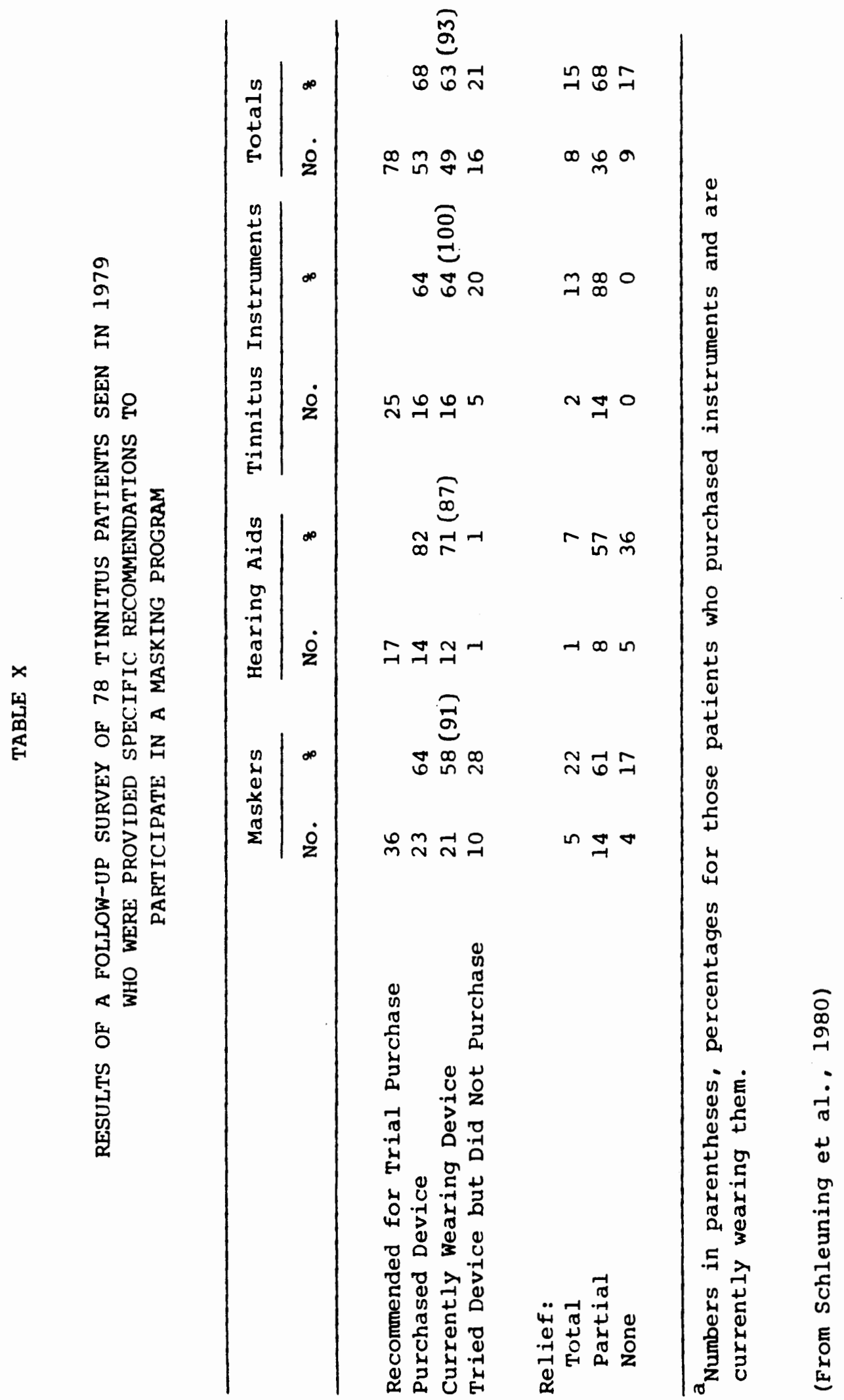


maskers at that time (Vernon \& Meikle, 1981; Schleuning et al., 1980). The results from all three types of masking available show more acceptable responses. Of a total of 380 recommended for trial purchase, 143 or $66 \%$ received some form of relief from tinnitus. Results from Group 2 are markedly better. Of those recommended for trial purchase of the masker $(36), 23$ or $64 \%$ did so and 21 or $94 \%$ are presently wearing them. Again, the results from the combined application of available types of maskers yielded an even greater response. Of the total of 78 recommended for trial purchase, 44 or $83 \%$ obtained some relief from their tinnitus.

As a result of these findings, Vernon and Meikle (1981) and Schleuning et al. (1980) have concluded that careful screening and evaluation, combined with appropriate application of tinnitus maskers can provide help for 60 to $80 \%$ of those suffering from clinically significant tinnitus.

From the above review of the literature, it should be evident that the vast amount of time and effort devoted to the study of tinnitus has uncovered no single etiology or cure for the problem. Instead, we are left with the overwhelming sense that we have only opened a Pandora's box, with the intricacies of the auditory and central nervous systems serving a worthy challenge to those who would attempt to unravel the mysteries that lie within, especially where tinnitus is concerned.

To this end, researchers have devoted their lives to the study of this intriguing phenomenon. Until recently, the only medical advice offered for those suffering from tinnitus was that they would have to live with it. With the advent of the tinnitus masker, a new approach to 
management of the tinnitus patient has become avallable. However, along with this new approach, several criticisms have been leveled at its application without sufficient prior research (Loavenbruck, 1980;Roeser \& Price, 1980), which cumulatively reflect the need for additional research with respect to its effects on the auditory and central nervous system.

Therefore, it is in the spirit and interest of scientific inquiry that this study has been proposed. Since only one study has been reported on the effects of tinnitus maskers on speech perception among normals (Spitzer et al., 1981), it is the intent of this investigation to test speech discrimination among tinnitus patients who currently wear tinnitus maskers. 


\section{CHAPTER III}

METHODS

\section{Subjects}

The subjects (Ss) comprising the experimental group for this study consisted of 26 adults ( 9 females and 17 males) ranging in age from 30 to 76 years, with a mean age of 51.7 years. They were selected from the files of tinnitus patients previously treated at the Tinnitus Clinic, Kresge Hearing Research Laboratory, Portland, Oregon. Only those Ss who met the following criteria were included in this study.

1) Subjects must have had speech discrimination scores of $50 \%$ or better, via sound field, at most comfortable listening levels (MCL).

2) Have a sensorineural hearing loss as measured by pure tone air conduction testing and tympanometry revealing normal compliance $(0.28$ to $2.5 \mathrm{cc}$ ) and middle ear pressure (not to exceed $150 \mathrm{~mm}$ $\mathrm{H}_{2} \mathrm{O}$ ) for the tinnitus ear(s). If an air-bone gap was suspected, 1.e., Type B or C tympanogram, pure tone bone conduction testing was to be used to screen out Ss with conductive or mixed losses.

3) Tinnitus was required to be present at the time of testing as judged by the $S$.

4) Subject must have owned the tinnitus masker(s) for at least 3 months.

The Ss were grouped according to the type of instrument worn. The initial proposal was to investigate the effects on speech discrimination utilizing two masking devices, a tinnitus instrument (Audiotone TA-641) and a tinnitus masker (Audiotone T-570 and T-520). However, due to the small number of people wearing only tinnitus maskers $(T-570$ and $T-5<0)$ 
in the Portland geographical area, the Ss for this study were divided in the following manner. Group I consisted of thirteen Ss wearing the TA-641 tinnitus instrument, a combination hearing aid and tinnitus masker $(T-570)$. Group II was comprised of thirteen Ss, nine of whom wore the TA-640 tinnitus instrument, which is also a combination hearing aid and tinnitus masker $(T-520)$, while the remaining four Ss were wearing maskers only $(T-520)$. Although the initial proposal was to include data only for the T-520 masker, an identical masking circuit was provided in a more readily available instrument, the TA-640. Therefore, data derived from Ss wearing this instrument were included in the treatment by treatment design.

\section{Equipment and Materials}

All preliminary data were obtained in a double-walled Industrial Acoustics Company (IAC) test suite ${ }^{1}$, located at the Kresge Hearing Research Laboratory, Portland, Oregon. Pure tone air conducted stimuli for octave intervals 250 through $8,000 \mathrm{~Hz}$ were generated by a dual-channel Grason Stadler audiometer (Model 1701) and transduced by a matched set of Telphonics earphones (Model TDH-49) mounted in foam rubber Acoustic Research cushions (Model MX-41 AR).

Speech test material consisted of sixteen lists of prerecorded NU-6 monosyllabic words (Auditec of St. Louis), Lists 1 through 4, Forms A

1For reasons of convenience and accessibility, this location was chosen as the test sight, even though the test chamber contained hard-walled surfaces used in a previous study. It was felt that the short distance from the speaker combined with the favorable signal-to-noise ratio adequately compensated for most of the reverberent qualities of this chamber. 
through $D$. These materials were presented under sound field conditions utilizing a reel-to-reel AKAI tape recorder (Model $4000 \mathrm{DB}$ ), coupled to the above-mentioned audiometer. A prerecorded portion of calibrated $( \pm$ $1 \mathrm{~dB}$, V.U.) cafeteria noise served as the competing noise signal and was presented using a Sharp stereo cassette recorder (Model RT-10) coupled to the same audiometer. Spectral analysis of the cafeteria noise was conducted utilizing a Tektronix spectrum analyer (Model 5L4N) with the resultant wave form being viewed on a Tektronix oscilloscope (Model 5103N). A Polaroid picture of the noise was taken using a Tektronix oscilloscope camera (Model C-27) and can be seen graphically in Figure 14. Speech and noise signals were presented at a signal to noise ratio $(S / N)$ of $5 d B( \pm 2 d B)$ and transduced through a Grason Stadler speaker placed at a 0 degree azimuth to the $S$, at a distance of one meter.

Tinnitus matching data for pitch and loudness were obtained using a Norwest Acoustics tinnitus synthesizer (Model SG-1), coupled to a set of Koss earphones (Model Pro/AAA) designed to provide optimum frequency response from 2,000 to $15,000 \mathrm{~Hz}$. Impedance measurements on all Ss were made using an American Electromedics impedance bridge (Model 86 AR).

The tinnitus instruments used in this study were those regularly worn by the Ss and are available commercially through the Audiotone Corporation. The three models chosen for inclusion were as follows: 1) an over-the-ear HFE (high frequency emphasis) tinnitus masker (Model T-520) with a frequency response range of 3000 to $15,000 \mathrm{~Hz}$ and a maximum power output of $80 \mathrm{~dB} S \mathrm{SL}, 2$ ) an over-the-ear tinnitus instrument (Model TA-641) which incorporates a T-570 masker with a wideband frequency response range of 1000 to $15,000 \mathrm{~Hz}$ and a maximum 


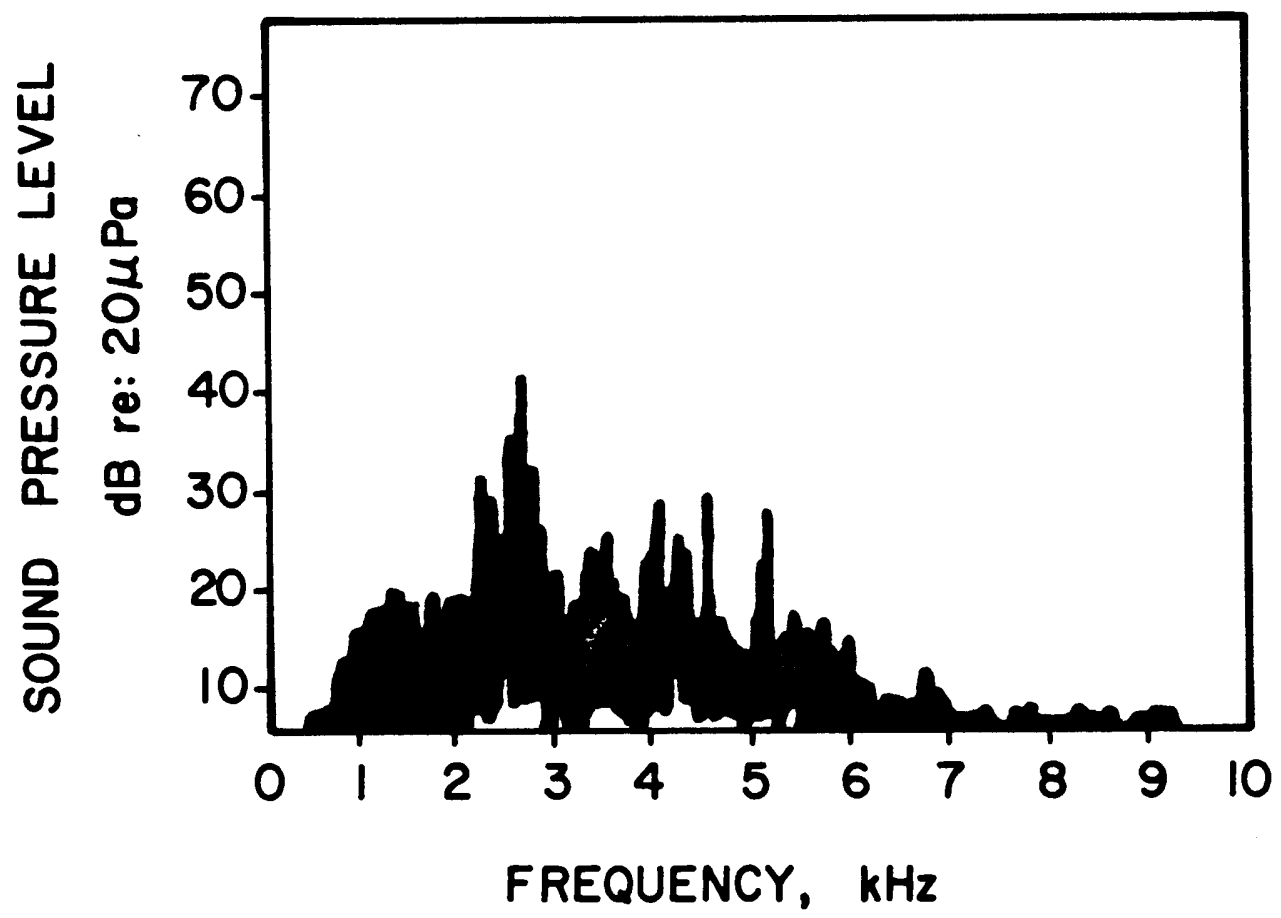

Figure 14. Graphic representation of a spectral analysis of the cafeteria noise utilized in this study. 
power output of $90 \mathrm{~dB}$ SPL, and 3) an over-the-ear tinnitus instrument (Model TA-640), which incorporates a T-520 masker (see Figures 15 and 16). Both the TA-641 and TA-640 instruments contain a hearing aid (Audiotone, A-31) which has a frequency response range of 415 to $6800 \mathrm{~Hz}$ and a maximum power output (MPO) of $126 \mathrm{~dB}$ SPL. Al though the se masking devices are designed with certain specific applications in mind, there is considerable overlap in the frequency regions, with noise bands containing energy throughout much of the frequency spectrum (see Figures 15 and 16).

Discrimination material used in this study consisted of the Northwestern University Auditory Test $\$ 6$, designed by Tillman and Carhart (1966). It was chosen on the basis of its high degree of intertest reliability and the quality of research behind its development.

\section{Calibration}

All audiometric equipment was calibrated to reflect current specifications prescribed by the American National Standards Institute (ANSI) standards. Calibration of earphones, linearity of the attenuator, and frequency measurements were made prior to the study, three times during the experimental sessions, and after all ss had been tested. Calibration of the tinnitus analyzer/synthesizer consisted of a frequency evaluation and a linearity check of the attenuators. Since all tinnitus loudness levels were made in sensation levels, exact measurements of sound pressure levels for the analyzer were not necessary. 

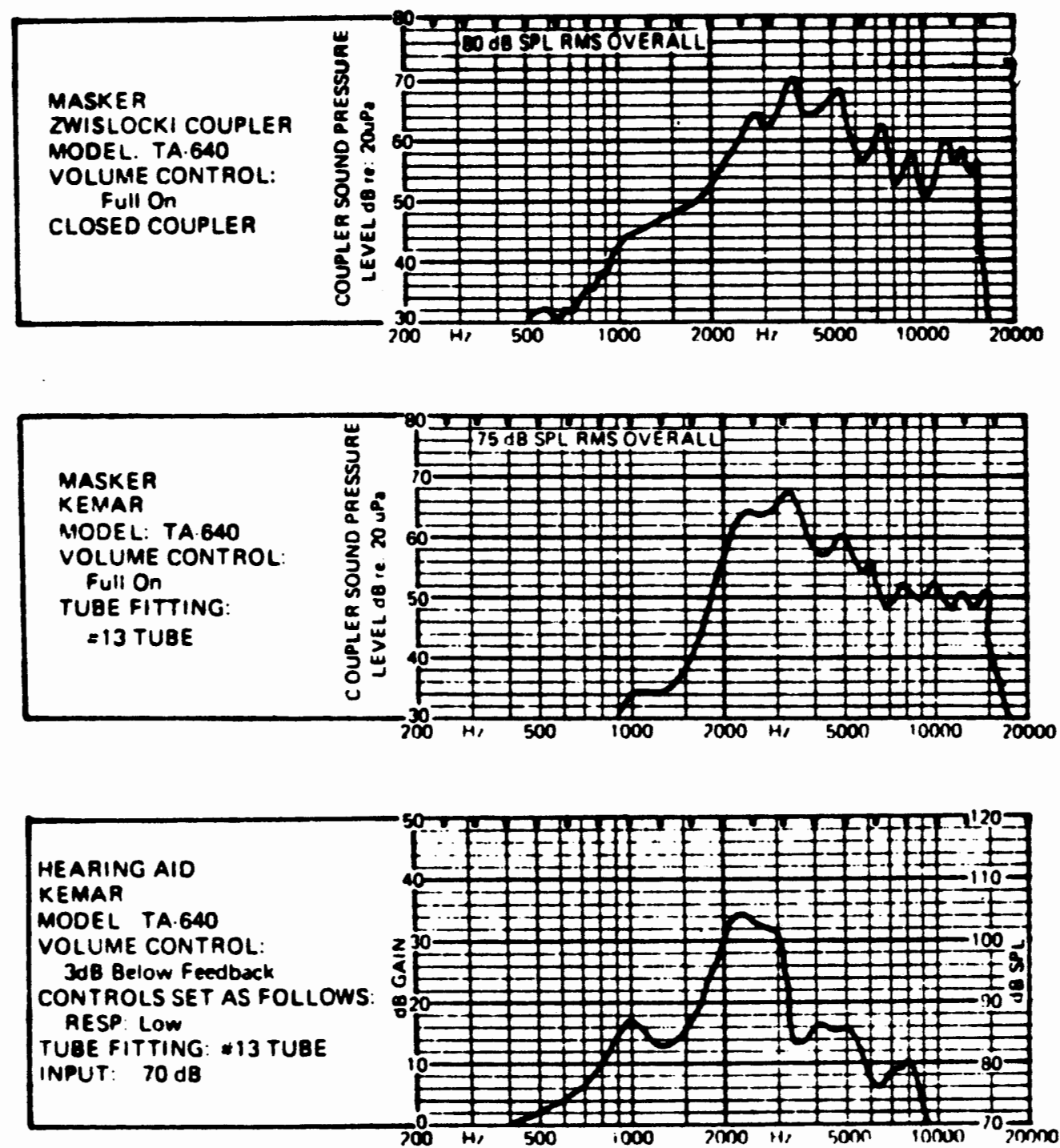

Figure 15. Frequency response curves of the TA-640 tinnitus instrument (From Audiotone's specifications on performance data for this instrument) 

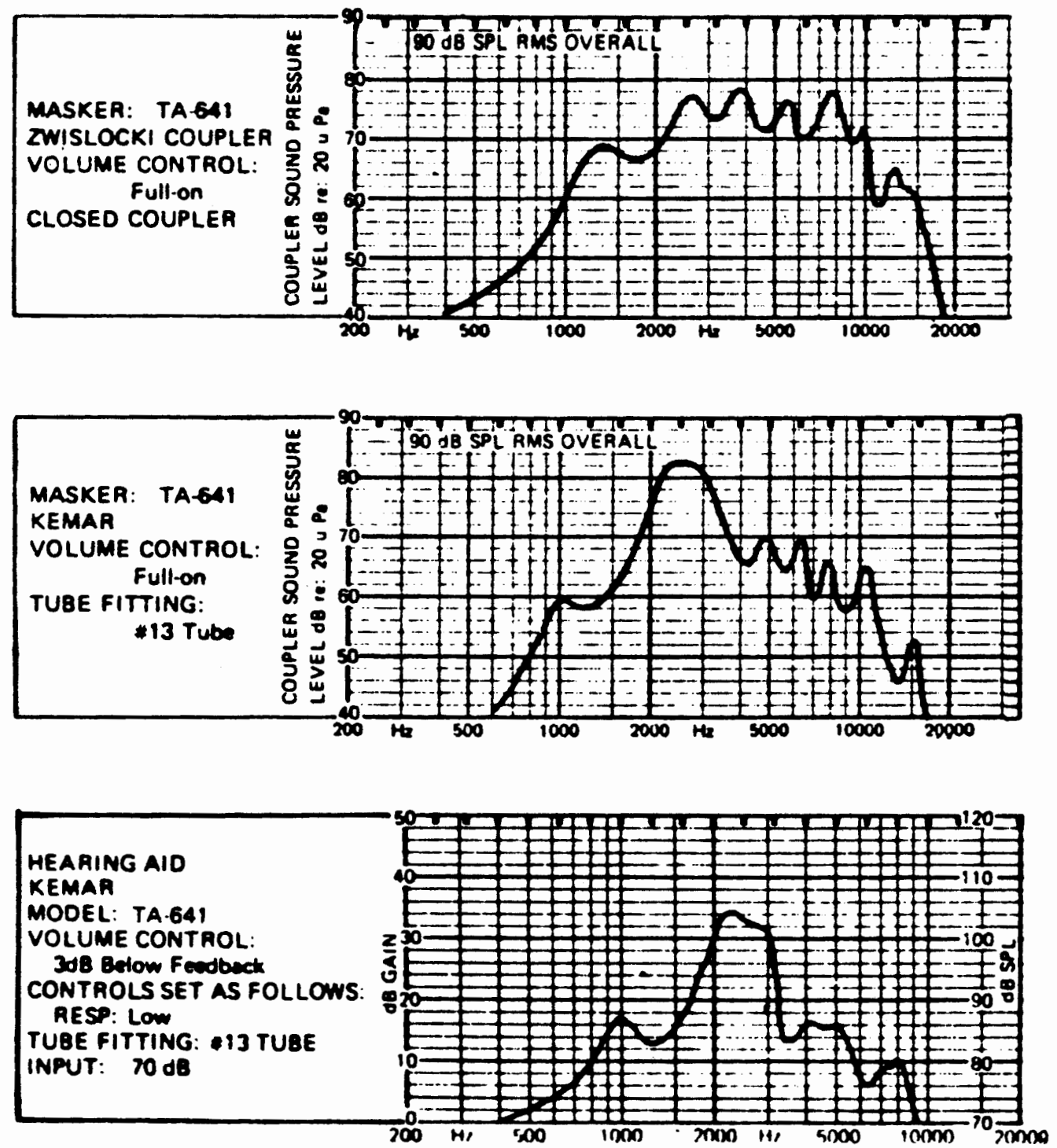

Figure 16. Prequency response curves of the TA-641 tinnitus instrument

(From Audiotone's specification on performance data for this instrument) 
The loudspeaker was callbrated in the following manner, as prescribed by Wilber (1978). Speech spectrum noise was transduced through the speaker and measured with a $B$ \& $K$ sound level meter (Model 2209) placed in a position approximating the center of the Ss head during testing, as well as at distances of 6 and 12 inches to the left and right of center. All measurements were made at a level of $80 \mathrm{~dB} \mathrm{HL}$ and at a distance of 1 meter.

\section{Procedures}

Prior to testing, each $S$ completed a special questionnaire related to subjective effectiveness of their tinnitus maskers (see Appendix A). Each $S$ was then administered an audiologic evaluation consisting of the following tests: 1) pure-tone air conduction test (at octave intervals 250 through $8,000 \mathrm{~Hz}$ ) (see Figure 17), 2) impedance testing (tympanometry and compliance to show Type $A$ and peak readings within $\pm 150 \mathrm{~mm} \mathrm{H}_{2} \mathrm{O}$ ), and 3 ) speech discrimination testing at MCL (NU-6 lists via sound field). Any Ss showing evidence of existing middle ear pathology, through abnormal impedance results, were tested by pure tone bone conduction to establish the sensorineural acuity levels. Any Ss complaining of aural pain or exhibiting an air-bone gap greater than 10 $d B$ HL for any two consecutive frequencies were excluded from further participation and referred to their physician.

The frequency and loudness of each individual's tinnitus was evaluated in a manner modeled after a technique developed at the Tinnitus Clinic, and is described as follows: 


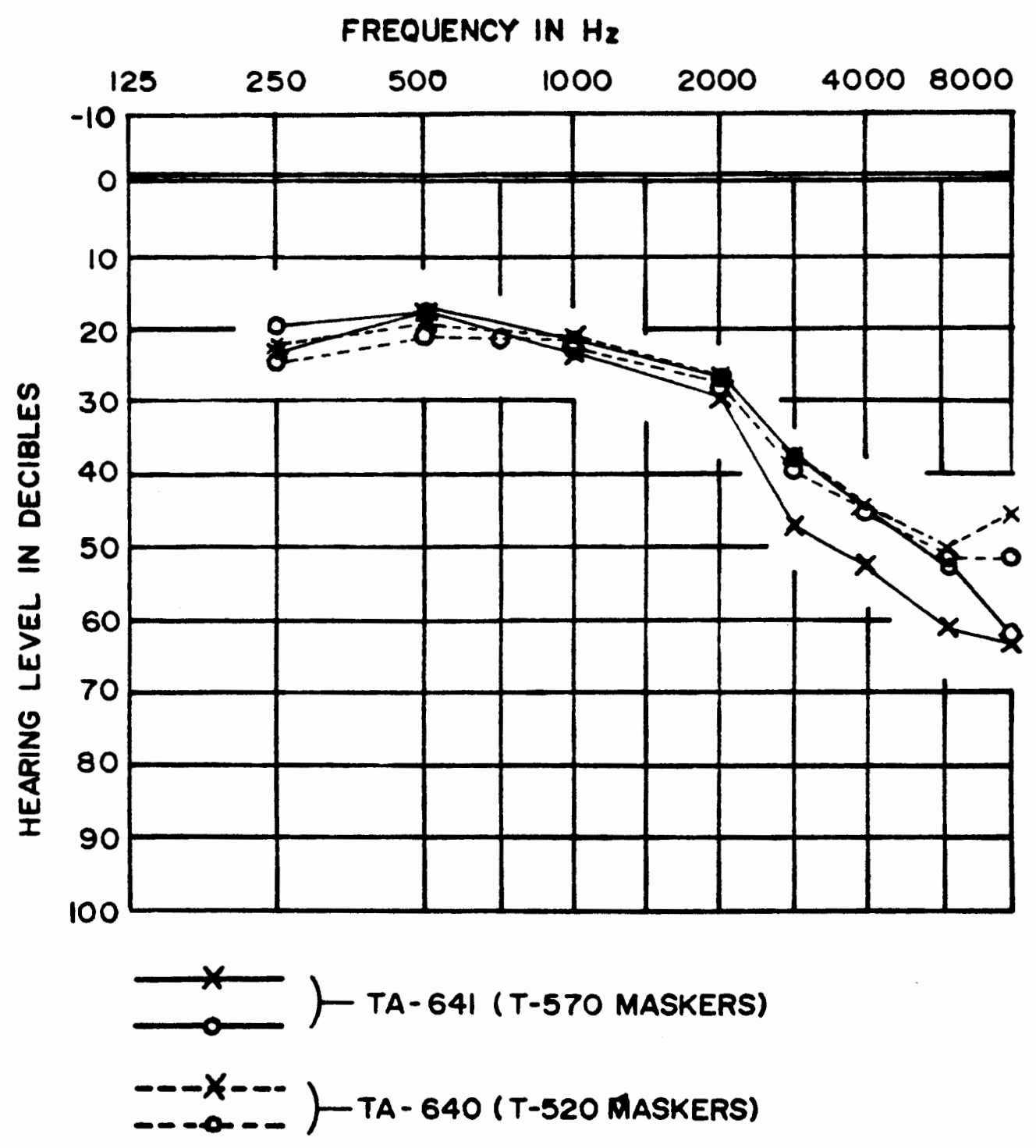

Figure 17. Mean pure tone air conducted threshold responses for 26 listeners who wear tinnitus instruments. 


\section{Erequency match}

1) S read instruction sheet $\# 1$ (see Appendix B) as experimenter read them aloud.

2) In the ear opposite that of the tinnitus, or the least involved, a $1000 \mathrm{~Hz}$ tone was presented through Channel $I$ and increased in intensity until the $S$ just heard 1 t.

3) The intensity was then increased $5 \mathrm{~dB}$ from this level.

4) With the $1000 \mathrm{~Hz}$ tone turned off, a $2000 \mathrm{~Hz}$ tone was presented through Channel II and increased in intensity until the $S$ just perceived 1 t.

5) The intensity was then increased $5 \mathrm{~dB}$ from this level.

6) The two tones were then slowly turned on and off, alternating between each frequency.

7) The $S$ was asked to determine which of the two tones was more like his tinnitus.

8) If the higher frequency was chosen, the frequency in Channel I was increased to $3000 \mathrm{~Hz}$.

9) The threshold level was then determined for the new frequency and set at $5 \mathrm{~dB}$ SL.

10) Steps 5 and 6 were repeated, substituting the new frequencies as necessary (moving to lower frequencies, if needed).

11) This procedure was continued until the $S$ indicated that one frequency was most like the pitch of the tinnitus.

12) To check for octave confusions, one channel was set at an octave above that matched with the tinnitus, alternating between the tinnitus frequency and the octave interval.

13) The response was recorded as the final pitch for Trial 1.

14) This process was repeated until two of three pitch matches were obtained at the same frequency. 
15) This was recorded as the final pitch match for this S.

\section{Loudness Match}

1) S read instruction sheet $\$ 2$ (see Appendix B) as experimenter read them aloud.

2) In the ear opposite that of the tinnitus, the tone most closely matched to the frequency of the tinnitus above was raised in intensity beginning below the previously determined threshold, in $1 \mathrm{~dB}$ increments, until the tone was just audible. This dial setting (in SL) was recorded as Threshold $\# 1$.

3) The stimulus was then increased in $1 \mathrm{~dB}$ increments until the s indicated that the tinnitus and the external tone were equal in loudness. This level was recorded as Tinnitus Level $\$ 1$.

4) The difference between the threshold and tinnitus level was the loudness of the tinnitus in SL.

5) The above procedure was repeated three times and then averaged, thus constituting the loudness of the tinnitus.

After pitch and loudness measures were obtained, each $S$ read instruction sheet $\# 3$ (see Appendix B) as the experimenter read them aloud. The experimenter then presented taped speech stimuli consisting of NU-6 word lists via sound field at MCL. Each s adjusted his masker/aid to a user level prior to all speech testing. All speech stimuli were presented sound field either in quiet or in the presence of cafeteria noise at a signal-to-noise ratio $(S / N)$ of $+5 d B( \pm 2 d B)$. The two conditions of presentation in quiet and in cafeteria noise were counterbalanced and the order of presentation was randomized. Each subject was tested during a single session which lasted approximately $11 / 2$ hours.

The results are reported and discussed in the following chapter. 


\section{CHAPTER IV}

\section{RESULTS AND DISCUSSION}

The purpose of this study was to determine the effects of tinnitus maskers and tinnitus instruments on speech discrimination utilizing a population of subjects who have tinnitus and wear these devices. The hypothesis which guided this investigation states that there is no difference between discrimination scores with and without tinnitus maskers. In an attempt to test this hypothesis, speech discrimination scores were obtained from 26 listeners ( 2 groups of 13 each) who currently have tinnitus and wear these devices. Both groups were tested once without instruments (open ears), once with masking only, and once with masking plus amplification. Each trial occurred under quiet listening conditions and in the presence of cafeteria noise for a total of six trials. Four exceptions to this procedure occurred in Group II (TA-640) and are described in Chapter III of this manuscript. For convenience, the results of this investigation will be reported under the headings of the two devices worn, results with the TA-641 tinnitus instrument (with T-570 masker) and results with the TA-640 tinnitus instrument (with T-520 masker) in order to determine the main effect of each instrument. A third division will report on the combined scores of both groups in order to determine if any overall main effect of the masking was present. 


\section{Results with the TA-641 Tinnitus Instrument}

The mean speech discrimination scores obtained for this group are reported in Table 11 with individual scores displayed graphically in Figures 18 and 19. Inspection of these data reveals a considerable difference between the masker only vs. the no masker (open ears) condition in both quiet and noise. Closer inspection of Table 11 reveals that under the no masker condition in quiet, the mean performance score was $75.4 \%$, while the masker only mean score in quiet was $65.1 \%$; a difference of $10.3 \%$. It is also apparent from Table 11 that the use of amplification with masking improves the mean performance scores with a resul tant mean of $77.7 \%$, which is slightly greater than the mean open ears score obtained at MCL.

Similar results were observed for the competing noise condition. In the no masker condition, the mean score in noise was $56.2 \%$, while the masker only mean showed a decrease of $8.7 \%$, for an average score of 47.5\%. Again, the use of amplification with masking resulted in an improvement in mean performance, in this case, to $63.0 \%$.

The standard deviations for these speech discrimination scores are also reported in Table 11. The magnitude of performance variability for each condition appears to be quite large. The variances in the quiet condition range from $11.29 \%$ without masking to $17.29 \%$ with masking only, while the addition of amplification reduces variability to $11.54 \%$.

Under the noise condition, it can be seen that the variances are also quite large, ranging from $13.00 \%$ without masking to $16.08 \%$ with masking only. Again, use of amplification reduced the variability, in this case, to $12.21 \%$. 
TABLE XI

MEANS AND (STANDARD DEVIATIONS) FOR SPEECH DISCRIMINATION SCORES (IN PERCENT CORRECT) FO 13 LISTENERS WEARING THE TA-641 TINNITUS INSTRUMENT (T-570 MASKER) IN QUIET AND CAFETERIA NOISE $(S / N+6 \mathrm{dBHL})$

\begin{tabular}{|c|c|c|}
\hline \multirow{2}{*}{$\begin{array}{c}\text { MASKING } \\
\text { CONDITION }\end{array}$} & \multicolumn{2}{|c|}{ NOISE CONDITION } \\
\cline { 2 - 4 } $\begin{array}{c}\text { QITHOUT MASKER } \\
\text { (OPEN EARS) }\end{array}$ & $75.4 \% \quad(11.29)$ & $56.2 \% \quad(13.00)$ \\
\hline $\begin{array}{c}\text { WITH MASKER } \\
\text { ONLY }\end{array}$ & $65.1 \% \quad(17.29)$ & $47.5 \% \quad(16.08)$ \\
\hline $\begin{array}{c}\text { MASKER PLUS } \\
\text { AMPLIFICATION }\end{array}$ & $77.7 \% \quad(11.34)$ & $63.0 \% \quad(12.21)$ \\
\hline
\end{tabular}

TABLE XII

MEANS AND (STANDARD DEVIATIONS) FOR SPEECH DISCRIMINATION SCORES (IN PERCENT CORRECT) OF 13 LISTENERS WEARING EITHER THE TA-640 TINNITUS INSTRUMENT OR T-520 TINNITUS MASKER, IN QUIET AND CAFETERIA NOISE $(S / N+6 \mathrm{dBHL})$

\begin{tabular}{|c|c|cc|}
\hline \multirow{2}{*}{$\begin{array}{c}\text { MASKING } \\
\text { CONDITION }\end{array}$} & \multicolumn{3}{|c|}{ NOISE } \\
\cline { 2 - 4 } & QUIET & \multicolumn{2}{c|}{ NOISE } \\
\hline $\begin{array}{c}\text { WITHOUT MASKER } \\
\text { (OPEN EARS) }\end{array}$ & $87.4 \%(8.77)$ & $66.8 \%$ & $(12.77)$ \\
\hline $\begin{array}{c}\text { WITH MASKER } \\
\text { ONLY }\end{array}$ & $79.8 \%(20.77) \star$ & $59.2 \%$ & $(20.22) \star$ \\
\hline $\begin{array}{c}\text { MASKER PLUS } \\
\text { AMPLIFICATION }\end{array}$ & $86.4 \%(6.84) \star 2$. & $65.3 \%$ & $(9.49) \star \star$ \\
\hline
\end{tabular}

丸 TA-640 tinnitus instrument plus T-520 tinnitus masker

$\star \star \star ⿻$ TA-640 tinnitus instrument only 


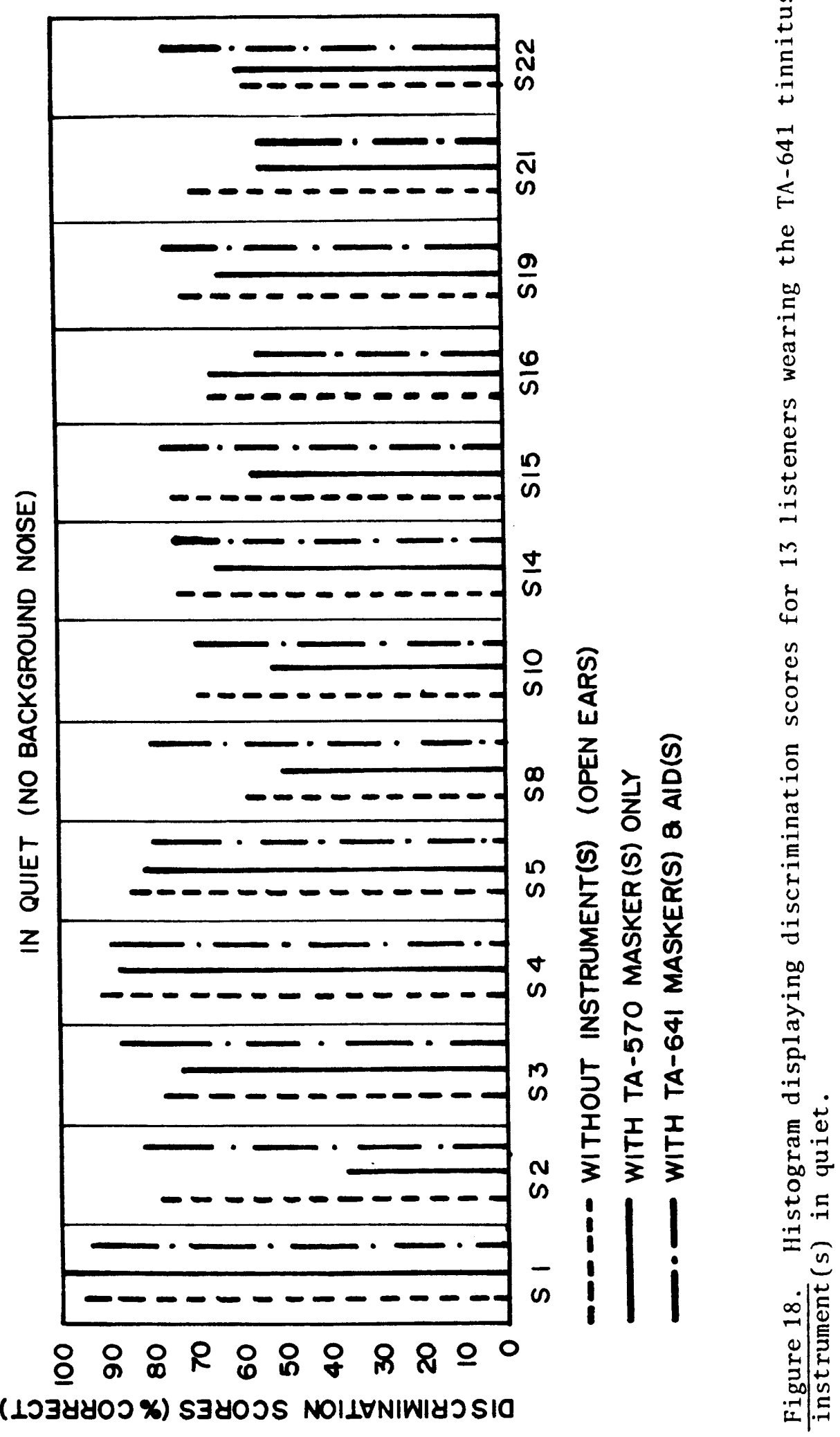




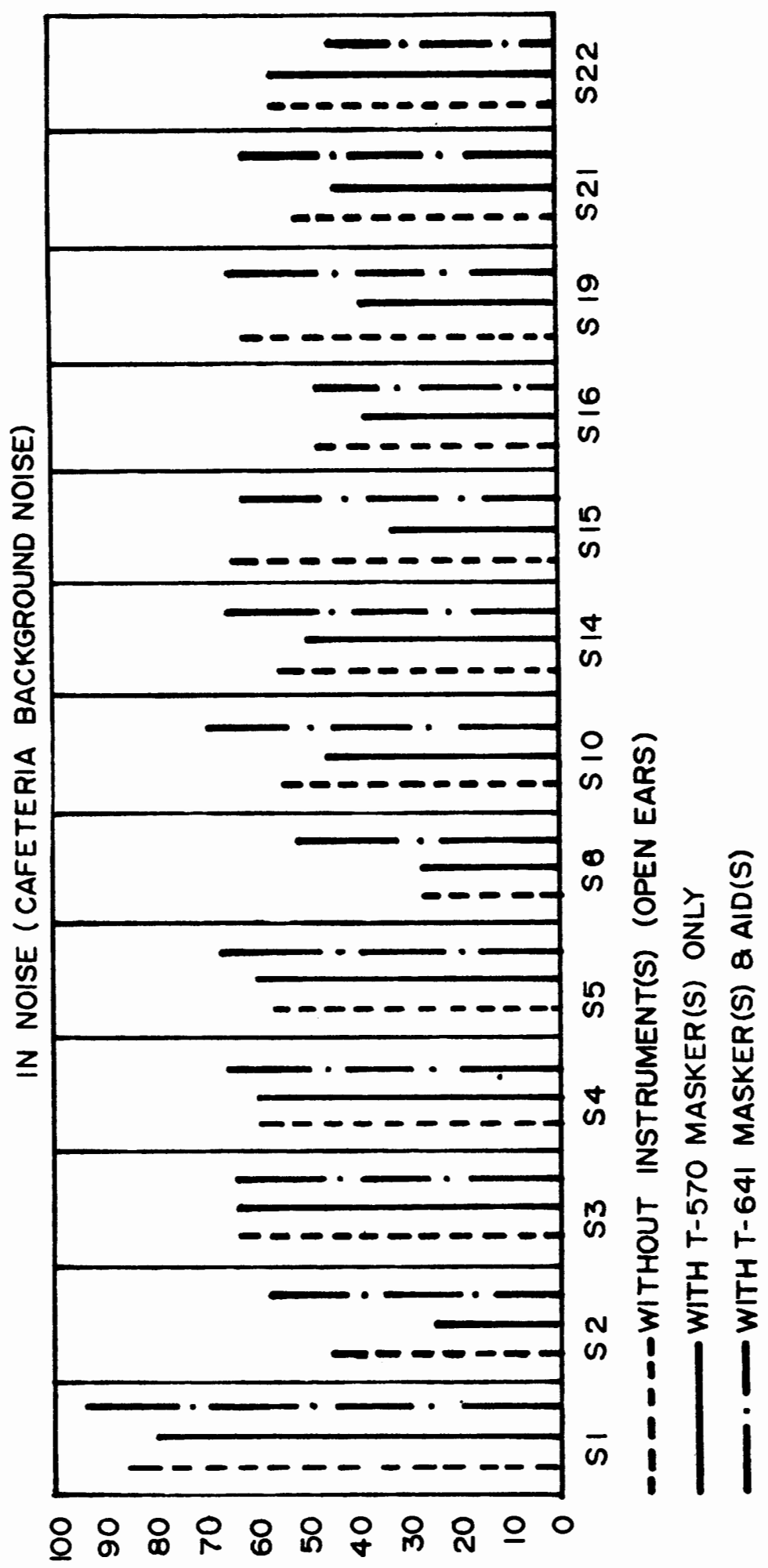

(1034800 \%) S3YOOS NOILVNIWIYJSIO

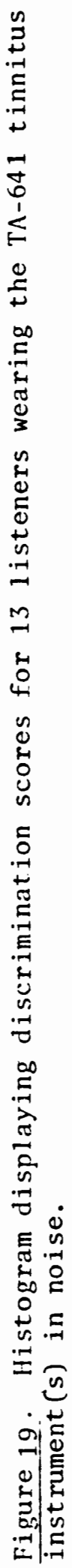


Further inspection of Table 11 reveals a general increase in variability scores with the addition of background noise, except in the masker only condition. It appears that background noise, (as has been shown in previous studies [Hirsh, 1952; Davis \& Fowler, 1960]) had a negative effect on speech intelligibility. It may be that the negative effect observed in this study was partially attributable to the overall variability in speech discrimination ability seen among the hearing impaired population. As an example, consider the extremely low scores of Subjects 2, 8, and 20, presented in Figures 18 through 21 . An examination of the scores for subject 20 indicates that the extreme variability occurring between the no masker/masker only condition in quiet (55-60\% difference) was considerably greater than the variability for the same condition in noise ( $45 \%$ difference), with the noise scores considerably lower in most cases. It is also important to note that in the majority of cases, the use of amplification with masking equalled or improved performance for all other conditions. This is important, because without amplification many of the subjects in this study would have experienced considerable difficulty communicating while using their masker(s) under these listening conditions.

In order to derive a more meaningful interpretation of the discrimination scores obtained for this group, the data were subjected to a more rigorous statistical analysis through the use of a multivariate analysis of variance (MANOVA) utilizing the statistical Package for the Social Sciences (SPSS) provided by Hull \& Nie (1981). Within this procedure the univarite option was selected to perform the analysis and a treatment by treatment by subject over repeated measures 
design was utilized.

The results indicate that the discrimination scores obtained under the masking only condition, both in quiet and noise, were significantly reduced from the other two conditions. The within group comparison of effects between the masker only/no masker (open ears) condition in quiet and noise were significant beyond the .05 level of confidence. Similar effects were observed when comparing the masker only/masker plus amplification condition with significance at the .005 level in quiet and .001 level in noise. This appears to indicate that the compared difference scores for the masker only/masker plus amplification has more significance than the masker only/no masker comparison. This implies that the addition of amplification has a beneficial effect on discrimination ability in conjunction with masker use. Comparing the no masker/masker plus amplification results with the above findings tends to support this implication, as would be expected, in that significance is not reached either in quiet or noise under these masking conditions.

Therefore, it appears that in Group I, discrimination ability is significantly reduced while masking is employed both in quiet and noise, unless amplification is used in conjunction with masking.

\section{Results with the TA-640 Tinnitus Instrument}

Before reporting the results of this group, it should be noted that four of the thirteen subjects tested wore only the T-520 tinnitus masker. Therefore, no data are available under the masking with amplification condition for these four subjects.

The mean speech discrimination scores for this group are reported in Table 12 with the individual scores displayed graphically in Figures 
20 and 21. Once again, the reader will note the large disparity between the masker only vs. the no masker (open ears) conditions. Under the no masker condition, the mean score in quiet was $87.4 \%$, while the masker only mean score was $79.8 \%$, a difference of $7.6 \%$. Further inspection of Table 12 reveals that the addition of amplification improved the mean score to $86.4 \%$, which is comparable to the no masker score.

Similar results were observed for the competing noise condition. The no masker mean score in noise was $66.8 \%$, while the masker only mean score decreased to $59.2 \%$, a difference of $7.6 \%$. Once again, the addition of amplification improved the mean discrimination score, in this case, to $65.3 \%$. However, the overall improvement observed with the addition of amplification was not as great as that observed in the TA-641 instruments. This difference may be attributable to a host of contributing factors, and will be discussed later.

Examining Table 12, it can be seen that the standard deviations for this group were also quite large. Variability scores for the quiet condition ranged from 8.77 with open ears to 20.77 with masking only. Again, the addition of amplification in conjunction with masking markedly reduced the variability. In this case, variances were reduced almost threefold, with the use of amplification, to 6.84. Under the competing noise condition, variances ranged from 12.77 without masking, to 20.22 with the masker only, and 9.49 with the addition of amplification. The reader will also note that the noise condition produced the greatest variability except in the masker only condition, which remains essentially unchanged from the quiet condition. 


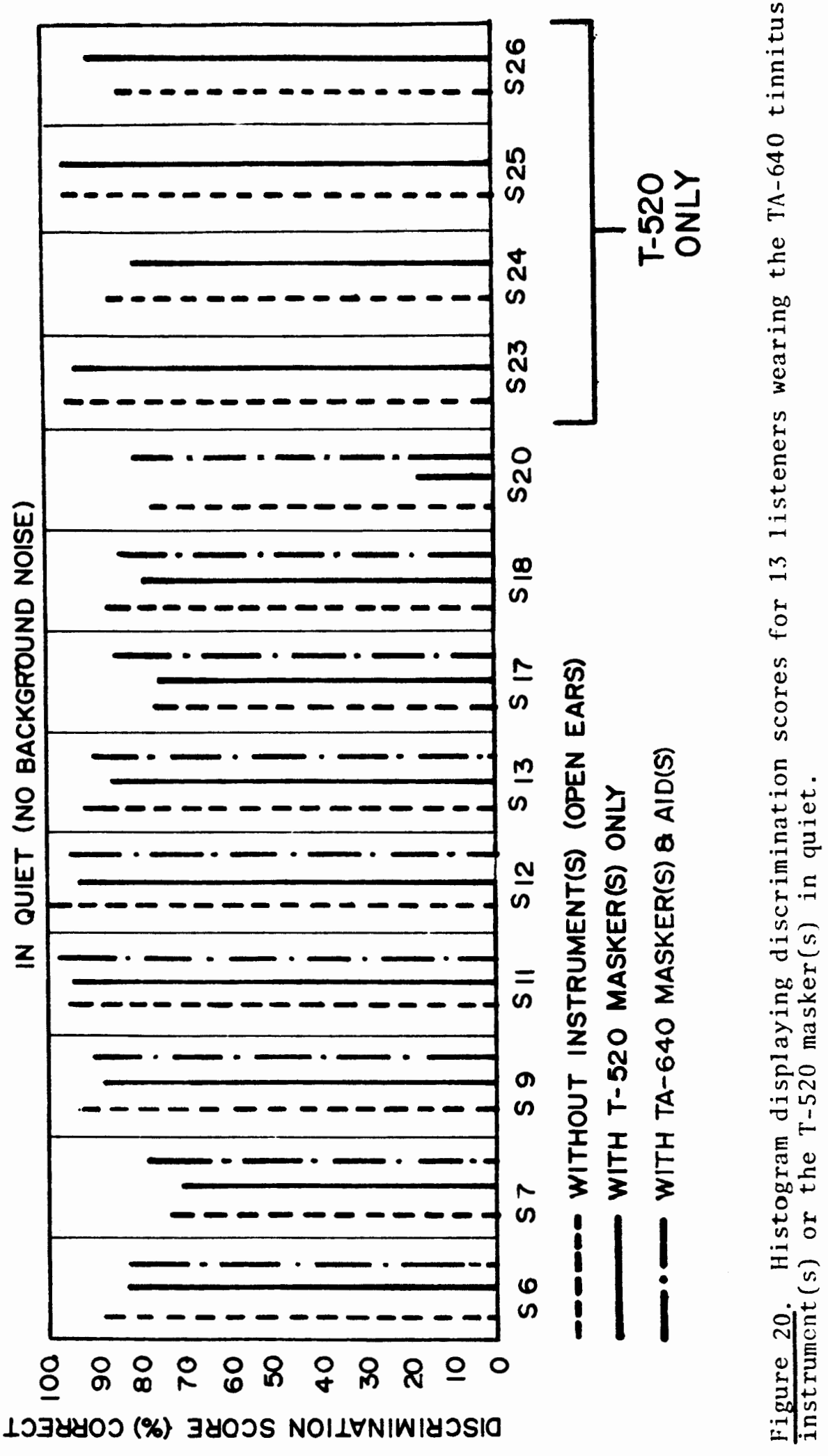




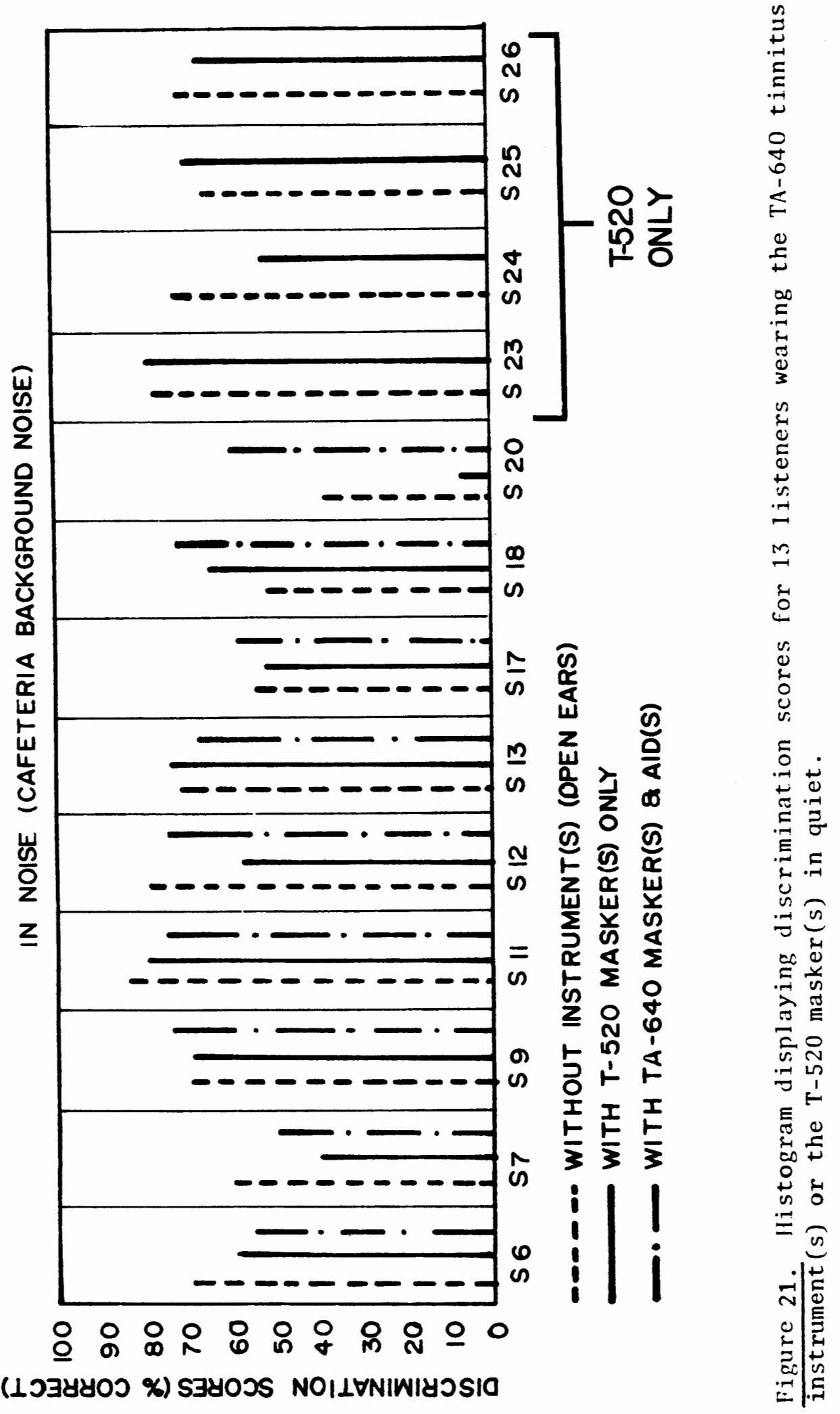


Examination of Figure 22 offers the reader a graphic comparison of the means and standard deviations displayed in Tables 11 and 12, and discussed above. It is apparent from this figure that there is a general disparity between the mean discrimination scores observed with the two types of instruments used in this study under the various testing conditions. Overall performance scores for the TA-640's were higher than its newer counterpart, the TA-641, in all cases.

Several factors may be contributing to these observed differences. First, the population size for each group must be considered. Group II was comprised of nine subjects wearing the TA-640 and four subjects wearing the T-520 only. It may be that this difference was significant enough to produce the observed disparity in performance. However, close inspection of Figures 18 to 21 reveals that in only four instances did any of Group II's performance scores ( $N=13$ total) fall below 50\% either with or without masking in noise and in quiet. Performance scores for Group I, however, fell below 50\% in 11 instances under these two masking conditions. This would tend to contraindicate any negative effect of population size differences.

Another possible factor which may be contributing to the observed disparity is the differing electroacoustic characteristics of each instrument. Looking at the overall mean performance scores, it appears that the TA-640 group out-performed its successor, the TA-641. Inspection of Figures 15 and 16 reveals that the frequency spectrum of the noise for the TA-641 contains energy from approximately $1,000 \mathrm{~Hz}$ to $15,000 \mathrm{~Hz}$, whereas the masking energy for the TA-640 is confined to a higher frequency region (approximately $3,000 \mathrm{~Hz}$ to $15,000 \mathrm{~Hz}$ ), with a 
TINNITUS INSTRUMENT TA-641 TINNITUS INSTRUMENT TA-640

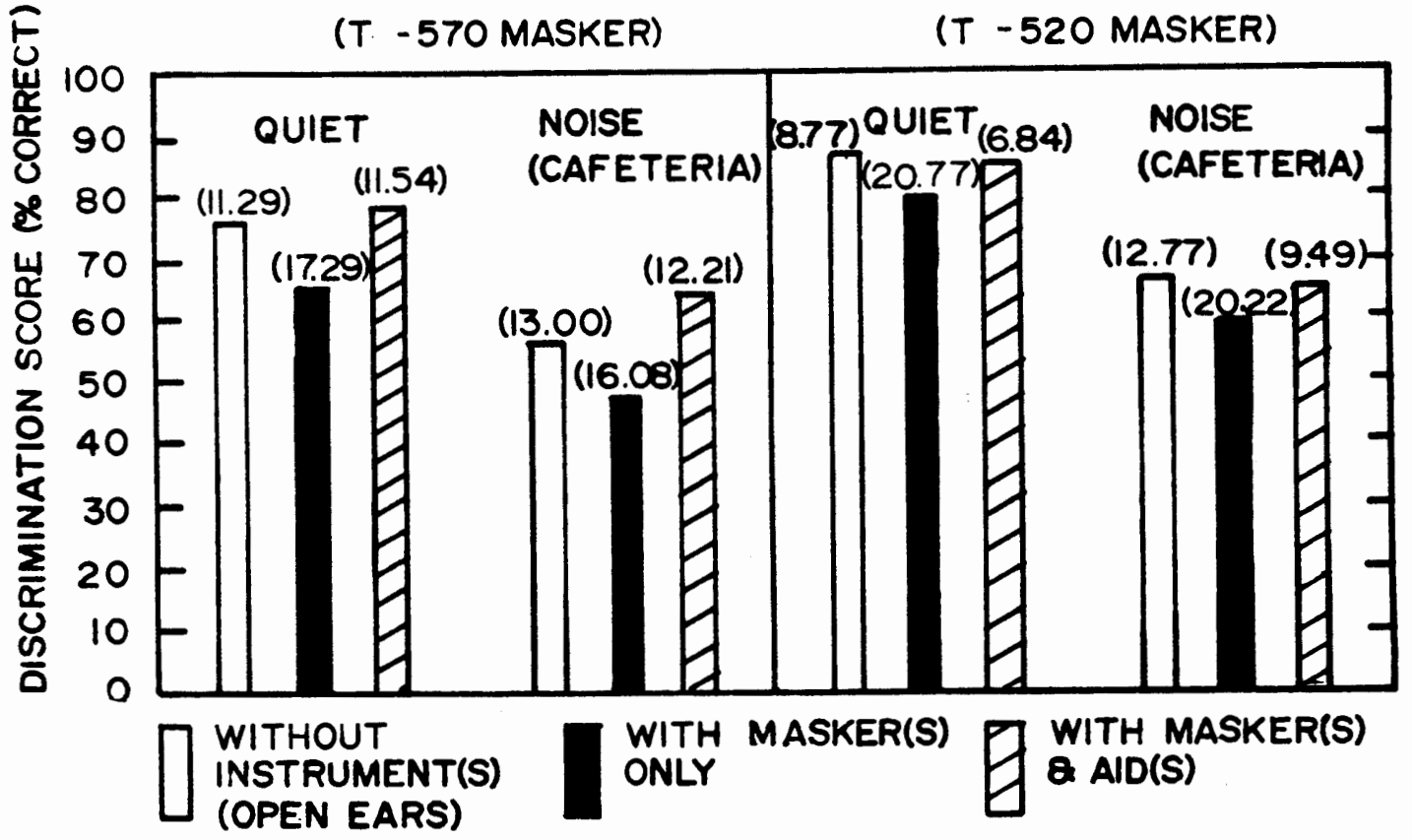

Figure 22. Histogram comparing means and (standard deviations) for speech discrimination scores of 26 listeners wearing either the TA-641 or TA-640 tinnitus instrument $(s)$ in quiet and cafeteria noise. 
reduced output of approximately $10 \mathrm{~dB}$ SPL across these frequencies. Perhaps the TA-641 has sufficient energy at $1 \mathrm{kHz}$ and below to produce generally poorer discrimination scores as a result of masker invasion of critical speech frequencies.

As a third possibility, age might also be a contributing factor in performance variability. Subjects comprising Group I have a mean age of 56 years, while Group II's subjects have a mean age of only 48 years. Numerous authors (Gaeth, 1948, Glorig \& Davis, 1961; Schuknecht, 1955; Willeford, 1978) have documented the deleterious effects of age on the auditory system, in particular, speech discrimination. Their results, although at times conflicting, are in general agreement that age does indeed have a profound and somewhat varied effect on speech discrimination ability. Maurer and Rupp (1979), after citing studies by Feldman and Reger (1967), Blumenfeld, Bergman and Millner (1969), Carhart and Nicholls (1971), and Mayer (1975), documenting the disadvantages of the elderly while listening in a noisy environment, expand upon this by taking a more realistic approach stressing that reduction in performance potential can begin in the 50-60 year range for most aging individuals.

As with the TA-641 group, further statistical analysis through use of the MANOVA was utilized to test for main effects among this group. Interpretation of the data indicate that the reduced discrimination scores under the masking conditions, both in quiet and noise, failed to reach significance for this group.

The within group comparison of effects between the masker only/no masker (open ears) condition were not significant beyond the .135 and 
.227 levels in quiet and noise, respectively. Additionally, comparison of the effects observed between the masker only/masker plus amplification condition were not significant beyond the .134 and .185 levels in quiet and noise. Perhaps the factors suggested above (population size, age and electroacoustic characteristics) played a mediating role in this group's results as well. However, the lack of any significant effect on discrimination ability suggests that the narrow band masker does not substantially interfere with discrimination for these subjects. Therefore, the reader must consider this a strong possibility. Indeed, Group II's overall performance was superior to Group I's, and the use of the TA-640 tinnitus instruments and T-520 maskers had no significant effect on speech intelligibility for the subjects tested. How much of this may be due to age is open for conjecture.

\section{Combined Scores}

After examining the intelligibility scores for each group separately and assessing the performance characteristics of each, it appears that a trend is manifesting itself in several respects. First of all, in no case was the mean score under masking only greater or equal to the no masking (open ears) or masking plus amplification conditions (see Figure 22). Secondly, addition of background noise degraded speech intelligibility in all cases. It is common knowledge that background noise interferes with the ability to understand speech for most normal listeners (Hirsh, 1952; Davis \& Fowler, 1961). It is also known that for the hearing impaired, background noise reduces speech discrimination ability even further (Ross, 1978; Tillman \& 
Carhart, 1971). Add to this the combined effects of age and additional noise from the maskers themselves and it seems evident from Tables 11 and 12 and Figure 22 that this particular population would have more difficulty under conditions of background noise with the use of masking. It is also evident from these same tables and figures that the addition of amplification under these conditions improved performance in all but six cases.

The reader will also note in Figure 22 an interesting effect observed in the noise condition for both instruments. In both groups the addition of cafeteria noise appeared to decrease the variance somewhat more in the no masker conditions when compared to the no masker quiet condition. However, the variances were consistently lower for the remaining conditions in quiet for both groups. This tends to support the findings of past research that noise from any source can interfere with speech intelligibility.

In an effort to determine if there is an overall main effect present, the combined scores from both groups were subjected to the same MANOVA procedure described above. Results from this analysis indicate that the reduced discrimination scores obtained under the masking only condition were significant, both in quiet and noise.

The combined comparison of the effects between the masker only/no masker condition were significant at the .01 and .05 levels of confidence for quiet and noise, respectively. Similar effects were observed for the masker only/masker plus amplification condition, with significance beyond the .005 level of confidence. As observed in Group I, this tends to Indicate that the difference in scores between the 
masker only/masker plus amplification condition has greater significance than the masker only/no masker scores. Therefore, it can be inferred that the addition of amplification in confunction with masking provided an overall beneficial improvement in discrimination ability. Again, comparing the no masker/masker plus amplification results to the above findings lends support to this observation in that significance is not reached either in quiet or noise, as was the case for Group I.

Overall, for the subjects in this study, there appears to be a significant deleterious effect on discrimination ability when tinnitus maskers are used either in quiet or in the presence of background cafeteria noise. However, when comparing the two types of instruments utilized in this study, there is a significant difference in the effects of each on discrimination ability. Group I (TA-641) showed significant effects on speech intelligibility while Group II (TA-640) did not.

Only one reported study (Spitzer et al., 1981) has previously assessed the effects of tinnitus maskers on speech discrimination ability. Comparison of their results with those of this study would be misleading for several reasons. First, the former study utilized a population of young adults with normal hearing. This differs from the present study in that only patients currently wearing masking devices were used, most of whom had a sensorineural hearing loss. Secondly, the types of devices used in the Spitzer study differed in some cases from those worn by the subjects in this investigation. Thirdly, the types of competing background noises used by the Spitzer group were white noise and cocktail party noise. The present study used only cafeteria noise as a competing stimulus. Differences in experimental design between the 
two studies also varied considerably.

There is, however, one data comparison that appears relevant to the Spitzer study. The means and standard deviations for their normal listeners were reported as $47.8 \%$ and $25.41 \%$ for the T-570 masker (the same type as used in this study), and $49.8 \%$ and $15.48 \%$ for the TM-3 masker (manufactured by Starkey Laboratories). Both sets of scores were obtained in cocktail party noise $(\mathrm{S} / \mathrm{N}=\mathrm{OdB} \mathrm{HL})$. Comparing these scores with those of the present study, the reader will note that the mean score for the masker only condition in noise for Group I of this study is only $0.3 \%$ less ( $47.5 \%$ ) than the mean score for Spitzer's group. Whether this is a result of the combined effects of masking and background noise in general, or merely coincidence, is left open for speculation. But one conclusion can be drawn from both studies, and that is despite somewhat different experimental procedures, this close agreement supports the concept that background noise can have a significant effect on speech intelligibility for both normals and hearing impaired listeners while wearing tinnitus maskers.

In that there are significant differences between the no masker and masker only conditions in both quiet and noise, the hypothesis for this investigation, which states that there is no difference between discrimination scores with and without maskers, must be rejected for the combined results and for Group I (TA-641 with T-570 masker). However, the hypothesis for Group II (TA-640 with T-520 masker) must be accepted. 


\section{CHAPTER V}

\section{SUMMARY}

The occurrence of tinnitus likely has plagued mankind since his earliest beginnings. It has been variously described as resulting from listening to one's own body sounds, to headaches, to evil spirits entering the body, to epilepsy. Treatment for these early diagnoses generally consisted of whatever local religious or superstitious customs were in existence at the time.

As man progressed and his knowledge of anatomy, physiology, and medicine grew, his desire to know more about the auditory system grew as well. From the dawn of modern medicine, theories describing the etiology of and providing treatment for tinnitus appear to parallel new developments in otology. Moving from the earlier theories which implicate the external and middle ear as the site of lesion of tinnitus, the turn of the century brought new interest to the inner ear and eventualiy the more central portions of the auditory pathway. The latter appears to have come full circle with some of the eariest described observations of tinnitus as a form of epilepsy, with recent studies implicating a site of lesion medial to the auditory periphery (Pennar, et al., 1981; Burns, 1982).

Through all these theories, diagnoses and remedies, one truth has prevailed, tinnitus has remained a serious health problem for millions of people. Until recently, the primary solution for most was 
reassurance from their doctor that nothing could be done medically and that they would have to learn to live with their problem. The advent of the electronic hearing aid reduced the annoyance of tinnitus for many sufferers, and the development of the first maskers (Vernon, 1977) provided additional relief for many of those not amenable to treatment with amplification. With the successful application of a masking device utilizing an external tone to mask tinnitus, a new means of treating tinnitus patients became available.

The following years brought new advances in hearing aid technology and tinnitus maskers became popular as an alternative, and in some cases a more successful, means of treating tinnitus. However, as more clinics began using the masker, questions as to the reliability and effectiveness of these devices began to be raised (Loavenbrack, 1980; Roser \& Price, 1980).

It seemed evident that in light of the many questions raised and the lack of agreement from the audiologic and otologic community concerning the effectiveness of tinnitus maskers, that further research was necessary to delineate the effects of these devices on the auditory system. Therefore, the present study was designed in an attempt to determine the effect tinnitus maskers may have on speech discrimination within a sample population who wear these devices.

\section{Conclusion}

This investigation was designed to test speech discrimination among two groups of subjects who have tinnitus and wear tinnitus maskers. The results indicate that in Group I (TA-641 with T-570 masker), discrimination scores were significantly reduced both in quiet and in the 
presence of cafeteria noise when masking was employed. Furthermore, when amplification was used with the masking, discrimination scores improved dramatically to near maximum intelligibility or greater with open ears. Analysis of Group II discrimination scores under open ears and masking conditions in quiet and noise revealed no significant masking effect. That is, the maskers did not appear to significantly effect discrimination ability among this group. Additionally, when the two group's scores were combined and that data analyzed, a significant masker effect was observed in both quiet and noise. Again, the use of amplification improved performance scores and reduced the magnitude of any masking effect beyond any level of statistical significance.

The foregoing results tend to indicate that the TA-641 tinnitus instrument had a deleterious effect on speech discrimination when masking was used. The TA-640 tinnitus instrument, however, appeared to out-perform its successor $(T A-641)$ in all respects. That is, this particular group showed no evidence of any significant effect on speech intelligibility when masking was employed.

\section{Implications for Further Research}

The results of this study indicate several areas for further research. It appears evident that a more sensitized measure of discrimination testing is warranted when evaluating a tinnitus patient for fitting with tinnitus maskers. Continued study of tinnitus patients wearing maskers at periodic intervals to determine Iong-range effects of maskers on discrimination ability would provide valuable information regarding the efficacy and acceptability of these devices. The use of an articulation curve may prove useful in identifying those individuals 
not suitable for masking due to poor discrimination ability with masking, even though tinnitus may be maskable. Further studies should be conducted in various other nolse conditions which may aid in the selection of appropriate maskers or combination masker and amplification systems for use by the tinnitus patient.

Also, in order to compare optimal aided and unaided discrimination ability, with and without masking, discrimination testing with amplification only should be added to the three masking conditions tested in this study. 


\section{REFERENCES}

Ambrosino, S.V., "Neuropsychiatric Aspects of Tinnitus." In Tinnitus: Breceedings of the First International Tinnitus Seminar (N. Y., June 8-9, 1979), Suppl. 4, Jeurnal of Laryngology and Otology, 169-172, 1981 .

The American Heritage Dictionacy of the English Language, Houghton, Mifflin, Co., Boston, 1968.

Anderson, J.R., Zoller, H.J., and Alexander, L.W., nobservations on the Treatments of Deafness and Tinnitus with Parenteral Vitamin A in Massive Doses." Eye. Ear. Nese and Throat Menthly, 29:75-79, 1950.

Atherly, G., Hempstock, R., and Noble, W., "study of Tinnitus Induced Temporarily by Noise." JASA, 44:1503-1506, 1968.

Atkinson, M., "Tinnitus Aurium." Annals ef Otology, Rhinology \& Laryngelogy, 55:398-405, 1944.

Atkinson, M., "Vitamin A Treatment of Tinnitus and Chronic Progressive Deafness." Archives of Otolaryngology, 59:192-194, 1954.

Baron, S.H., "Experience with Parenteral Vitamin A Therapy in Deafness and Tinnitus." Lacyngoscope, 61:530-547, 1951.

Bau, H.W., \& Savitt, L., "Treatment of Chronic Progressive Deafness and Tinnitus with Massive Doses of Vitamin A." Eye.-Ear, Nese\& Throat Monthly, 30:83-86, 1951.

Blumenfeld, V., Bereman, M., and Millner, E., "Speech Discrimination in an Aging Population." JSHR, 12:210, 1969.

Burns, E., "Variability Measurements of Subjective Tinnitus." A research project presented at the Fifth Midwinter Research Meeting of the Association for Research In Otolaryngology. St. Petersburg, Florida, January 18-21, 1982.

Carhart, R., and Nicholls, S., "Perceptual Masking in Elderly Women." ASHA, 13:535, 1971 .

Cazals, Y., Negrevergne, M., and Aran, J.-M., "Electrical Stimulation of the Cochlea in Man: Hearing Induction and Tinnitus Suppression." Jeurnal of the American Audielogical society, $3(5)$ :209-213, 1978. 
Davis, H., and Fowler, E. In H. Davis \& S. Silverman, (Eds); Hearing and Deafness, Holt, Rinehart \& Winston, Inc., 1960.

Douek, E., and Reid, J, "The Diagnostic Value of Tinnitus Pitch." Journal of Laryngolegy and Otology, 82:1039-1042, 1968.

Durrant, J.D., and Lovrinic, J.H., Bases of Hearing Sciences, Baltimore: Williams and Wilkins, Co., 1977.

Emmett, J.R., \& Shea, J.J., "Diatrizoate Meglumine (Hypaque) Treatment for Sudden Hearing Loss." In Tinnitus: Proceedings of the Finst International Tinntus Seminar, (N.Y., June 8-9, 1979), Suppl. 4, Journal of Laryngology and Otolosy, 139-143, 1981.

Engstrom, H., \& Graf, W., "Recording of Objective Tinnitus." Acta etolaryngolesica, 41:228-34, 1952 .

Feldman, A., \& Reger, S., "Relation among Hearing, Reaction Time and Age." JSHD, 10:479-495, 1967.

Feldmann, H., "Homolateral and Contralateral Masking of Tinnitus by Noise-Bands and by Pure Tones." Audiology, 10:138-144, 1971.

Flottorp, G., \& Wille, C., "Nicotinic Acid Treatment of Tinnitus." Acta etolaryngologica, Suppl. 18, 85-99, 1955.

Fowler, E., "Tinnitus Aurium--Its Significance in Certain Diseases of the Ear." New York State Je of Medicine, 12(12):702-704, 1912.

Fowler, E.P., nHead Noises: Significance, Measurement and Importance in Diagnosis and Treatment." Archives of Otolaryngology, 32: $903-914,1940$.

Fowler, E.P., "Tinnitus Aurium in Light of Recent Research." Annals of otolegy. Rhinelesy \& Laryngelogy, 50:139-148, 1941.

Fowler, E.P., "The Illusion of Loudness of Tinnitus--Its Etiology and Treatment." Lacyngoscope, 42:275-285, 1942.

Fowler, E.P., "Head Noises in Normal and Disordered Ears." Archiyes of Otol aryngolesy, 39:498-503, 1944.

Fowler, E.P., "Nonvibratory Tinnitus: Factors Underlying Subaudible and Audible Irritations." Archives of Otolaryngology, 47:29-36, 1948.

Fowler, E.P., "Subjective Head Noises." Laryngoscope, 75:1610-1618, 1965.

Fowler, E.P., \& Fowler, E.P., Jr. "An Explanation of Certain Types of Tinnitus and Deafness." Laryngoscope, 919-930, 1950. 
Gaeth, J., "A Study of Phonemic Regression Association with Hearing Loss." Unpublshed Doctoral Dissertation, Northwestern University, 1948.

Gerber, S., \& Mencher, G., "Adventitious Hearing Impairment." In S. Gerber \& G. Menscher (Eds.), Auditory Dysfunction, College-Hill Press, Houston, 1980.

Glorig, A., \& Davis, H., "Age, Noise and Hearing Loss." Annals ofotology. Rhinolegy and Laryngelegy, 70:556-571, 1961.

Goodhill, V., "The Management of Tinnitus." Laryngoscode, 60:442-450, 1950.

Goodhill, V., "A Tinnitus Identification Test." Annals of Otology, $61: 778-788,1952$.

Goodhill, V., "Tinnitus: New Concepts in Etiology and Management: Otologic Aspects." Iransactions of the American Academy of Oothalmolegy and Otolaryngology, 58:529-532, 1954.

Goodwin, P., \& Johnson, R., "A Comparison of Reaction Times to Tinnitus and Nontinnitus Frequencies." Ear and Hearing, 1(3):148-155, 1980.

Graham, J.T., "Tinnitus Aurium." Acta Oto-lanyngolegica, Suppl. 202, 1965.

Graham, J., \& Newby, H., "Acoustical Characteristics of Tinnitus." Archives of Otolaryngology, 75:82-87, 1962.

Grossan, M., "Treatment of Subjective Tinnitus with Biofeedback." Ear. Nose and Throat Journal, 55:314-318, 1976.

Hamblen-Thomas, C., "Physical Aspects of Tinnitus." Journal of Laryngelegy and Otolegy, 53:68-78, 1938.

Hatton, D.S., Erulkar, D.D., \& Rosenberg, P.E., "Some Preliminary Observations on the Effects of Galvanic Current on Tinnitus Aurium." Laryngescope, 70:123-130, 1960.

Hellar, M.F., \& Bergman, M., "Tinnitus Aurium in Normally Hearing People." Annals of Otology, Rhinology \& Laryngology, 62:73-83, 1953.

Hirsh, I., The Measurement of Hearing. New York: MoGraw-Hill, 1952.

House, J.W., "Treatment of Severe Tinnitus with Biofeedback Training." California Ear Research Institute and Otologic Medical Group, Inc., 1977 .

Hull, C., \& Nie, N., SPSSUpdate 7-9. New York: McGraw-Hill, 1981. 
Johnson, L.F., "Surgery of the Sympathetic in Meniere's Disease, Tinnitus Aurium and Nerve Deafness." Acchives of etolaryngology, 59:492-498, 1954 .

Jones, T.H., \& Knudsen, V.0., "Certain Aspects of Tinnitus, Particularly Treatment." Laryngescope, 38:597-611, 1928.

Josephson, E.M., "A Method of Measurement of Tinnitus Aurium." Archiyes of Otolaryngology, 14:283-284, 1931.

Kemp, D.T., "Stimulated Acoustic Emissions from Within the Human Auditory System." JASA, 64(5): 1386-1391, 1978.

Kennedy, A., "Cochlear, Neural and Subjective Factors in Tinnitus." Proceedings of the Royal Society of Medicine, $46: 829-831,1953$.

Lempert, J., "Tympanosympathectomy." Acchives of 0tolaryngelegy, 43:199-212, 1946.

Loavenbruck, A., "Tinnitus Masking Devices: Safe \& Effective?" ASHA, $22(10): 857-861,1980$.

Lobel, M.J., "Clinical Studies with Parenteral Vitamin A Therapy in Deafness: Preliminary Report." Eye, Ear, Nose \& Throat Monthly, 28:213-218, 1949 .

Loeb, M. \& Smith, R., "Relation of Induced Tinnitus to Physical Characteristics of The Inducing Stimul1." JASA, 42(2):453-455, 1967.

Lothrop, D., "The Presentation of a Theory Explaining a Phase of Tinnitus Aurium." Laryngoscope, 33:582-584, 1923.

Marlowe, F.I., "Effective Treatment of Tinnitus Through Hypnotherapy." American Journal of Clinical Hyonosis, 15:162-165, 1973.

Maurer, J., \& Rupp, R., Hearing and Aging: Tactics for Interyention. New York: Grune \& Stratton, Inc., 1979.

Mayer, C., "The Effects of Aging on the Discrimination of Speech in Noise." ASHA, 27, 653, 1975.

Meikle, M.B., Personal communication, Kresge Hearing Research Laboratory, Portland, Oregon, January, 1983.

Melding, P.S., Goodey, R.J., \& Thorne, P.R., "The Use of Intravenous Lignocaine in Diagnosis and Treatment of Tinnitus." Journal of Laryngelogy and Otology, $92: 115-121,1978$.

Myers, D., "Tinnitus." Hospital Medicine, 55-64, May, 1975. 
Nodar, R., \& Graham, J., "An Investigation of Characteristics of Tinnitus Associated with Meniere's Disease." Archiyes of Otol aryngology, 82:28-31, 1965.

Passe, E.R., "Sympathectomy in Relation to Meniere's Disease, Nerve Deafness and itrnitus." Rroceedings of the Reval society of Medicine, 44:760-771, 1951 .

Pennar, M., Brauth, S., \& Hood, L., "The Temporal Course of the Masking of Tinnitus as a Basis for Inferring its Origin." JSHR, $24: 257-261,1981$.

Reed, G.F., "An Audiometric Study of 200 Cases of Subjective Tinnitus." Archives of Otolaryngology, 71:84-94, 1960.

Riddel1, W., "Tinnitus." Harper H2, tal Bulletin, 14:153-158, 1956.

Roeser, R., \& Price, D., "Clinical Experience with Tinnitus Maskers." Ear and Hearing, 1(2):62-68, 1980.

Ross, M., "Classroom Acoustics and Speech Intelligibility." In J a ck Katz (Ed.), Handbook of Clinical Audiolosy, Baltimore: Williams \& Wilkins, Co., 1978.

Saltzman, M., \& Ersner, M.S., "A Hearing Aid for the Relief of Tinnitus Aurium." Laryngoscope, 57:358-366, 1947.

Sasaki, C.T., Kauer, J.S., \& Babitz, L., " $D$ i f $f$ e $r$ e $n$ t $i$ a $I$ [14c]2-deoxyglucose Uptake After Differentiation of the Mammalian Auditory Pathway--A Model for Examining Tinnitus." Brain Research, 194:511-516, 1980.

Schleuning, A.J., Johnson, R.M., \& Vernon, J.A., "Evaluation of a Tinnitus Masking Program: A Follow-up Study of 598 Patients." Ear and Hearing, $1: 71-74,1980$.

Schnider, D.E., "The Growth Concept of Nervous Integration." Journal of Neryous and Mental Diseases, 105:124-148, 1947.

Schuknecht, H., "Presbycusis." Lacyngoscope, 65:402-419, 1955.

Seltzer, A.P., "The Problems of Tinnitus in the Practice of Otolaryngology." Laryngoscope, 57:623-631, 1947.

Shea, J.J., \& Emmett, J.R., "The Medical Treatment of Tinnitus." In Innitus: Proceedings of the First International Tinnitus Seminar, (N.Y., June 8-9, 1979), Suppl. 4, Journal of Lacyngology and Otolesy, 130-139, 1981 . 
Shulman, A., "Clinical Classification of Subjective Idiopatnic Tinnitus." In Tinnitus: Proceedings of the First Internatzonal Tinnitus Seminar, (N.Y., June 8-9, 1979), Suppl. 4, Journal of Lacyngology and Otology, 102-107, 1981.

Spaulding, J.A., "Tinnitus, with a Plea for its More Accurate MusicalNotation." Archives of Otolaryngolegy, 32:263-272, 1903.

Spitzer, J.B., Goldstein, B.A., Salzbrenner, L.G., \& Muellar, G., "Effe ct of Tinnitus Masker Noise on Speech Discrimination in Quiet and Two Noise Backgrounds." Paper presented at ASHA Convention, Los Angeles, Nov. 20-23, 1981.

Stevens, S.S., \& Davis, H., Hearing--Its Psychology and Physiology, New York: John Wiley and Sons, Inc., 1938.

Tillman, T., Carhart, R., \& 0lsen, W., "Hearing Ald Efficiency in a Competing Speech Situation." JSHR, 13:789-811, 1970.

Trowbridge, B.C., "Tympanosympathetic Anesthesia for Tinnitus Aurium and Secondary Otalgia." Acchives of Otolaryngology, 50:200-215, 1949.

Venters, R.S., "Discussion of Tinnitus: Subjective Tinnitus or Tinnitus Aurium." Broceedings of the Royal Society of Medicine. 46:825-829. 1953.

Vernon, J.A., "Tinnitus." Hearing Aid Journal, 28:13-26, 1975.

Vernon, J.A., "The Loudness(?) of Tinnitus." Hearing and Speech Action, 44:17-19, 1976.

Vernon, J.A., "The Study of Tinnitus." Kresge Hearing Research Laboratory, Department of Otolaryngology, Oregon Health Sciences University, Portland, Oregon, 1977.

Vernon, J., Schleuning, A., Odell, L., \& Hughes, F., " $A$ T $i$ n $i$ it $\mathrm{s}$ Clinic." Ear, Nose \& Throat Journal, 56:181-189, 1977.

Vernon, J.A., \& Schleuning, A.J., "Tinnltus: A New Management." Laryngoscope, 88:413-419, 1978.

Vernon, J., "Some Observations on Residual Inhibition." In M. Paparella \& W. Meyerhoff (Eds.), Sensorineural Hearing Less. Vertige and Tinnitus, Baltimore: Williams and Wilkins, 1981.

Vernon, J.A., \& Meikle, M.B., "Tinnitus Masking: Unresolved Problems." CIBA Foundation Symposium 85, Pitman Books, Ltd., London, 239-262, 1981.

Wegel, R., "A Study of Tinnitus."

Archives of Otolaryngology," 14:158-165.1931. 
Wilber, L.A., "Calibration of Pure Tone, Speech and Noise Signals." In J. Katz (Ed.), Handbook of Clinical Audiology, Williams \& Wilkins, Baltimore, 1978.

Willeford, J., "The Geriatric Patient." In P. Rose (Ed.), Audiological Assessment, Prentice-Hall, Inc., Engl ewood Cliffs, N.J., 1978.

Wilson, J.P., "Evidence for a Cochlear origin for Acoustic Re-emissions, Threshold Fine-structure and Tonal Tinnitus." Hearing Research, 2:233-252, 1980.

Zemlin, W., Speech \& Hearing Science, New Jersey: Prentice Hall, 1968. 


\section{APPENDIX A}

\section{MASKER QUESTIONNAIRE}

1. Does the masker cover up or reduce your tinnitus?

YES

$$
\text { NO }
$$

2. Approximately how far do you turn the volume control to get relief?

Min

$$
1 / 4
$$

$1 / 2$

$3 / 4$

Full

3. Do you find the masker noise more pleasant to listen to than your tinnitus?

YES

NO

4. Are you more relaxed while wearing the masker?

YES NO

5. What time of day do you find the masker most helpful?

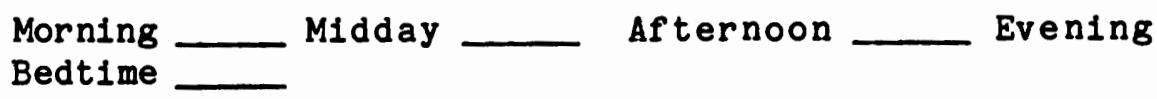

6. What is the average period of time you wear the masker daily?

Less than 1 hour $\quad 123456789910$ More hours

7. Do you notice any change in your tinnitus after you take your masker off?

Completely gone

Different Pitch

Softer Same

Louder

8. If you notice a change in your tinnitus after removing the masker, how long does it last?

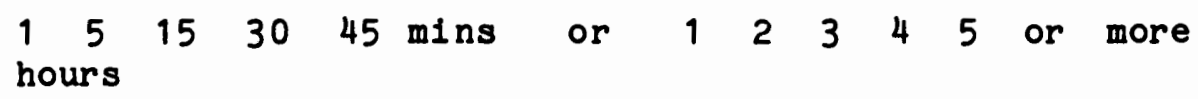

9. If you are wearing the masker on only one ear do you notice any change in the tinnitus in your other ear?

Completely gone

Softer

Louder

Different Pitch Same 
10. Do you use the masker to help you sleep?

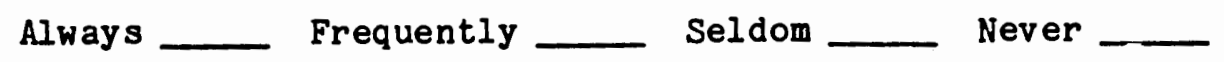

11. Do you feel that the masker intereres with your ability to understand speech in any way?

$$
\text { Very little } \begin{array}{rrrrrrr} 
& 1 & 2 & 3 & 4 & 5 & \text { A lot }
\end{array}
$$

12. Please comment on any experiences which you may have encountered with your masker(s) which are not included in this questionnaire. 
APPENDIX B

INSTRUCTIONS TO SUBJECTS

1. Erequency (Pitch) Matching Instructions

YOU ARE GOING TO HEAR SEVERAL PAIRS OF ALTERNATING TONES OF DIFFERING PITCH. YOUR TASK IS TO LISTEN TO EACH TONE AND DECIDE WHICH MOST CLOSELY MATCHES THE PITCH OF YOUR TINNITUS, THE EIRST OR SECOND TONE. WHEN YOU DECIDE, STATE "FIRST" OR "SECOND" SO I CAN RECORD YOUR RESPONSES.

2. Intensity (Loudness) Matching Instructions

YOU ARE GOING TO HEAR A TONE THAT SOUNDS LIKE THIS (tone presentation). I WANT YOU TO TELL ME WHEN YOU FIRST HEAR THE TONE. THEN I WANT YOU TO TELL ME WHEN THE TONE IS EQUAL IN LOUDNESS TO YOUR TINNITUS.

3. Speech Discrimination Instructions

YOU ARE GOING TO HEAR SEVERAL TAPE RECORDED WORDS. SOME WILL BE PRESENTED IN THE PRESENCE OF A BACKGROUND NOISE WHICH IS SIMILAR TO GARBLED SPEECH, AND OTHERS WILL BE PRESENTED WITHOUT THESE BACKGROUND NOISES. YOUR TASK IS TO LISTEN FOR AND REPEAT ONLY THE WORDS THAT ARE PRESENTED TO YOU. IF YOU ARE NOT SURE OF A WORD, PLEASE GUESS. 